University of Tennessee Health Science Center

UTHSC Digital Commons

\title{
$5-2011$
}

\section{Application of a High-Resolution Computed Radiography System in Detecting an lodinated X-ray Contrast Agent: Small Animal Phantom Study}

Anobel Maghsoodpour

University of Tennessee Health Science Center

Follow this and additional works at: https://dc.uthsc.edu/dissertations

Part of the Diagnosis Commons

\section{Recommended Citation}

Maghsoodpour, Anobel, "Application of a High-Resolution Computed Radiography System in Detecting an lodinated X-ray Contrast Agent: Small Animal Phantom Study" (2011). Theses and Dissertations (ETD). Paper 169. http://dx.doi.org/10.21007/etd.cghs.2011.0193. 


\title{
Application of a High-Resolution Computed Radiography System in Detecting an lodinated X-ray Contrast Agent: Small Animal Phantom Study
}

\author{
Abstract \\ Tumor angiogenesis, the creation of new blood vessels, is the characteristic of solid tumors and crucial \\ for their development. lodinated contrast agents are used to increase the X-ray detectability of zone of \\ angiogenesis, and thus providing a means for tracking tumor growth. \\ The overall objective of this project was to evaluate the performance of Kodak CR 7400, a high-resolution \\ compact computed radiography (CR) system in detection of Omnipaque-240, an iodinated contrast agent, \\ in a phantom mimicking small animal tumor model.
}

The first phase of the project was dedicated to a comprehensive assessment of CR image quality by measuring presampled Modulation Transfer Function (MTF), Noise Power Spectrum (NPS), Relative Standard Deviation of Noise (RSD), Noise Equivalent Quanta (NEQ), and Detective Quantum Efficiency (DQE).

Next, dual-energy and temporal subtraction techniques were implemented to enhance the contrast of iodinated regions and suppress soft tissue background in the phantom. The underlying physics of each technique was discussed, including the design of the phantoms, the simulation and measurement of the Signal-to-Noise Ratio (SNR) in the final subtracted iodine image, and dose assessment. In the end, the results of both techniques were compared along with discussions about the advantages and limitations of implementing each technique.

Overall, the study supported the potential of low-cost CR 7400 in small animal study, particularly detecting iodinated contrast agents implementing temporal subtraction technique and provided a background for similar small animal studies using a CR system.

\author{
Document Type \\ Dissertation \\ Degree Name \\ Doctor of Philosophy (PhD)

\section{Program} \\ Biomedical Engineering and Imaging

\section{Research Advisor} \\ Gary S. Keyes, Ph.D.
}

\section{Keywords}

Computed radiography, Contrast agent, Dual-energy subtraction technique, Small animal, Temporal subtraction technique, $\mathrm{X}$-ray projection

\section{Subject Categories}

Analytical, Diagnostic and Therapeutic Techniques and Equipment | Diagnosis | Medicine and Health Sciences 


\title{
APPLICATION OF A HIGH-RESOLUTION COMPUTED RADIOGRAPHY SYSTEM IN DETECTING AN IODINATED X-RAY CONTRAST AGENT: SMALL ANIMAL PHANTOM STUDY
}

\author{
A Dissertation \\ Presented for \\ The Graduate Studies Council \\ The University of Tennessee \\ Health Science Center \\ In Partial Fulfillment \\ Of the Requirements for the Degree \\ Doctor of Philosophy \\ In the Joint Graduate Program in Biomedical Engineering and Imaging \\ From The University of Tennessee \\ and \\ The University of Memphis
}

By

Anobel Maghsoodpour

May 2011 
Copyright (C) 2011 by Anobel Maghsoodpour. All rights reserved. 


\section{Acknowledgements}

Though only my name appears on the cover of this dissertation, different people have contributed to its production. I owe my gratitude to all who have made this dissertation possible.

My deepest gratitude goes to my research advisor, Dr. Gary Keyes for his guidance, insightful comments, and constructive criticism throughout different phases of this dissertation. I am so grateful to Dr. Herbert Zeman whose excellent technical knowledge and invaluable suggestions at different stages of my dissertation were critical for the completion of it. I also wish to extend my appreciation to Dr. Vivian Loveless for her help and support, as well as her enthusiasm and patience toward my research throughout the dissertation process. I am also grateful to Dr. Richard Smith, my graduate coordinator, who has been a mentor throughout my graduate career, and for always being so supportive. I would particularly like to thank Dr. Jinsong Huang, who has been very instrumental in finding materials necessary for my investigation, for his help in the subject matter, and for his friendship. I would also like to thank Joe Laughter for his assistance with the project set-up. Last not the least, I would like to appreciate Micheal Nguyen at the machine shop of the University of Tennessee Health Science Center, for his time and professional work on preparing the phantoms.

In the end, I would like to acknowledge the Society of Photo-Optical Instrumentation Engineers (SPIE) for their permission to use copyrighted figures.

This study was partially supported by an equipment grant from the Kodak Dental Systems, Carestream Health. 


\section{Abstract}

Tumor angiogenesis, the creation of new blood vessels, is the characteristic of solid tumors and crucial for their development. Iodinated contrast agents are used to increase the X-ray detectability of zone of angiogenesis, and thus providing a means for tracking tumor growth.

The overall objective of this project was to evaluate the performance of Kodak CR 7400, a high-resolution compact computed radiography (CR) system in detection of Omnipaque-240, an iodinated contrast agent, in a phantom mimicking small animal tumor model.

The first phase of the project was dedicated to a comprehensive assessment of CR image quality by measuring presampled Modulation Transfer Function (MTF), Noise Power Spectrum (NPS), Relative Standard Deviation of Noise (RSD), Noise Equivalent Quanta (NEQ), and Detective Quantum Efficiency (DQE).

Next, dual-energy and temporal subtraction techniques were implemented to enhance the contrast of iodinated regions and suppress soft tissue background in the phantom. The underlying physics of each technique was discussed, including the design of the phantoms, the simulation and measurement of the Signal-to-Noise Ratio (SNR) in the final subtracted iodine image, and dose assessment. In the end, the results of both techniques were compared along with discussions about the advantages and limitations of implementing each technique.

Overall, the study supported the potential of low-cost CR 7400 in small animal study, particularly detecting iodinated contrast agents implementing temporal subtraction technique and provided a background for similar small animal studies using a CR system. 


\section{Table of Contents}

Chapter 1 Introduction $\ldots \ldots \ldots \ldots \ldots \ldots \ldots$

Chapter 2 X-ray Imaging Background . . . . . . . . . . . . 3

2.1 X-ray Production . . . . . . . . . . . . . . . . . . 3

2.1.1 Fundamentals and Mechanisms . . . . . . . . . . . 3

2.1.2 Brehmsstrahlung Radiation . . . . . . . . . . . . . 4

2.1.3 Characteristic Radiation . . . . . . . . . . . . . . 5

2.2 X-ray Interactions with Matter $\ldots \ldots \ldots \ldots \ldots \ldots$

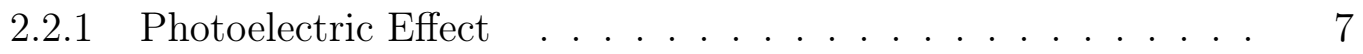

2.2 .2 Compton Scattering _. . . . . . . . . . . . . . . . 9

2.2 .3 Rayleigh (Coherent or Thomson) Scattering . . . . . . . 10

2.3 Attenuation Coefficients . . . . . . . . . . . . . . . 11

2.4 X-ray Detection . . . . . . . . . . . . . . . . . . . . 12

Chapter 3 Computed Radiography $(\mathrm{CR}) \ldots \ldots \ldots \ldots$

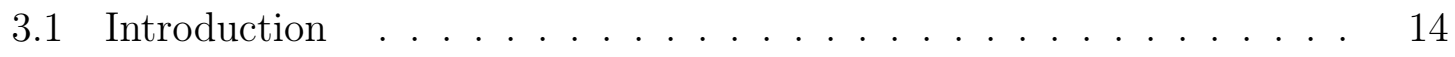

3.2 CR versus Screen $/$ Film . . . . . . . . . . . . . . . . . . . . 14

3.2 .1 Image Formation . . . . . . . . . . . . . . . . . . 14

$3.2 .2 \quad$ System Characteristics . . . . . . . . . . . . . . . . 19

3.3 Imaging Performance of $\mathrm{CR} \ldots \ldots \ldots \ldots$

3.3.1 Input/Output Relationship _ . . . . . . . . . . 20

3.3 .2 Spatial Resolution . . . . . . . . . . . . . . 20

3.3 .3 Contrast Resolution . . . . . . . . . . . . . . . . . 21

3.3 .4 Noise . . . . . . . . . . . . . . . . . . . . 21

3.4 Quality Improvement and Artifacts . . . . . . . . . . . . . . 22

3.5 New CR Developments . . . . . . . . . . . . . . . . . 22 
Chapter 4 Image Quality Assessment of a Computed Radiography

System ....................... 24

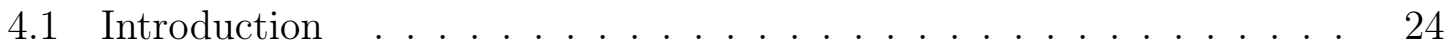

4.2 Imaging System . . . . . . . . . . . . . . . . . . 24

4.3 Exposure Geometry . . . . . . . . . . . . . . . . . . . . . . . 24

4.4 Exposure Conditions and Physical Characterization . . . . . . . . 26

4.5 Presampled Modulation Transfer Function (MTF) . . . . . . . . . 27

4.6 Noise Characteristics . . . . . . . . . . . . . . . . . . . . . . . . . . . . . 29

4.6.1 Noise Power Spectrum (NPS) _ . . . . . . . . . . . . . . . 29

4.6.2 Relative Standard Deviation (RSD) . . . . . . . . . . . . 29

4.6.3 Noise Equivalent Quanta (NEQ) _ . . . . . . . . . . . 30

4.7 Detective Quantum Efficiency $(\mathrm{DQE}) \ldots \ldots \ldots \ldots$

Chapter 5 Dual-energy Subtraction Technique . . . . . . . . . 32

5.1 Introduction . . . . . . . . . . . . . . . . . . 32

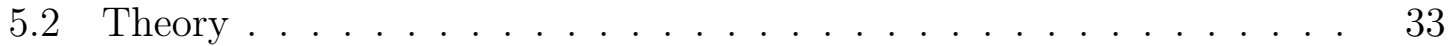

5.2 .1 Dual-energy Subtraction . . . . . . . . . . . . . . . 33

5.2 .2 Dual-energy Decomposition . . . . . . . . . . . . . . 34

5.3 Decomposition Calibration . . . . . . . . . . . . . . . 36

5.4 Decomposition Algorithms . . . . . . . . . . . . . . . . . . 38

5.5 Global Coefficient Algorithms . . . . . . . . . . . . . . . . 39

5.6 Test Phantom . . . . . . . . . . . . . . . . . . . . . . . . . 41

5.7 Computer Model . . . . . . . . . . . . . . . . . . . . . . . . . . . 41

5.7.1 Signal-to-Noise Ratio (SNR) in the Subtracted Iodine Image . 41

5.7 .2 Dose . . . . . . . . . . . . . . . . . . 42

Chapter 6 Temporal Subtraction Technique . . . . . . . . 45

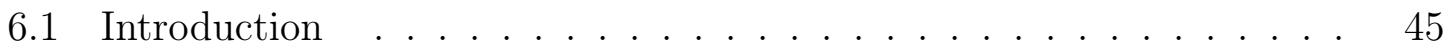

6.2 Theory . . . . . . . . . . . . . . . . . . 46

6.3 Test Phantom . . . . . . . . . . . . . . . . . . . . . . . 47

6.4 Computer Model . . . . . . . . . . . . . . . . . . . . . . . 47

6.4.1 Signal Difference-to-Noise Ratio (SdNR) . . . . . . . . 47

6.4 .2 Dose . . . . . . . . . . . . . . . . . . 47 
Chapter 7 Results and Discussion . . . . . . . . . . . 49

7.1 Image Quality Assessment of CR $7400 \ldots \ldots \ldots$

7.1 .1 Exposure Geometry . . . . . . . . . . . . . . . . . 49

7.1 .2 Response Curve . . . . . . . . . . . . . . . . . . . . . 49

7.1 .3 Presampled MTF . . . . . . . . . . . . . . . 49

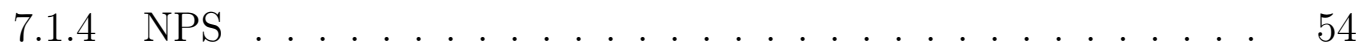

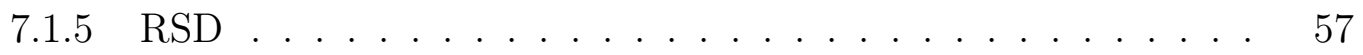

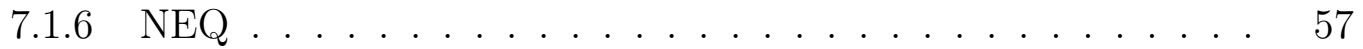

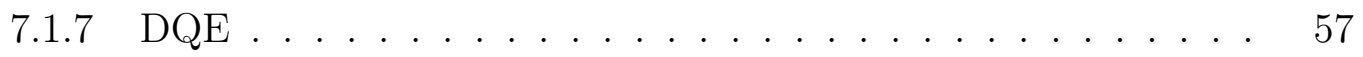

7.2 Dual-energy Subtraction Technique . . . . . . . . . . . 57

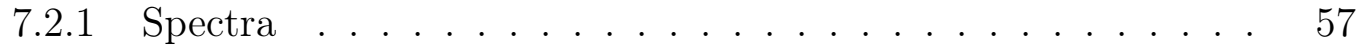

7.2 .2 Calibration Procedure . . . . . . . . . . . . . 61

7.2 .3 Test Phantom . . . . . . . . . . . . . . . . 63

7.2.4 Simulated SNR and Dose . . . . . . . . . . . 63

7.3 Temporal Subtraction Technique . . . . . . . . . . . . 70

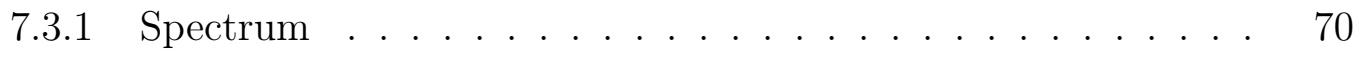

7.3 .2 Test Phantom . . . . . . . . . . . . . . . . . 70

7.3.3 Simulated SdNR and Dose _. . . . . . . . . . 70

7.4 Discussion of the Results . . . . . . . . . . . . . . . 70

Chapter 8 Conclusions $\ldots \ldots \ldots \ldots \ldots \ldots \ldots$

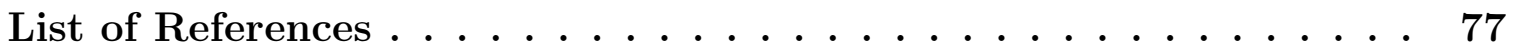

Appendix A Calibration Phantoms Drawings .........883

Appendix B Test Phantoms Drawings . . . . . . . . . . 86

Vita ......................... 89 


\section{List of Tables}

Table 4.1 CR 7400 specifications. . . . . . . . . . . . . . . 25

Table 7.1 Parameters for the MTF sixth-degree polynomial fit at 50- and 100-micron resolutions. . . . . . . . . . . . . . 54

Table 7.2 RSD analysis: values of the main noise components for CR 7400. . 57

Table 7.3 Calibration coefficients for the iodine and plexiglas surfaces. . . . . 63 


\section{List of Figures}

Figure 2.1 X-ray tube schematic. . . . . . . . . . . . . . . . 4

Figure 2.2 Brehmsstrahlung. . . . . . . . . . . . . . . . . . . 5

Figure 2.3 Characteristic X-ray production. . . . . . . . . . . . . 6

Figure 2.4 Photoelectric effect. . . . . . . . . . . . . . . . . . 8

Figure 2.5 Compton scattering. . . . . . . . . . . . . . . . . 10

Figure 2.6 Log-scale plots of mass attenuation coefficients contributed by the three main interactions for soft tissue (a), iodine (b), and barium (c). . . . . . . . . . . . . . . .

Figure 3.1 CR three-step imaging cycle: expose, readout, and erase. . . . . . 16

Figure 3.2 Storage phosphor stimulation curve. . . . . . . . . . . . . . 17

Figure 3.3 Storage phosphor emission and stimulation spectra. . . . . . . . . 17

Figure 3.4 Main components of a flying-spot CR scanner. . . . . . . . . . . . 18

Figure 4.1 The effect of penumbral blurring with respect to source-to-object and object-to-detector distances. . . . . . . . . . . 26

Figure 4.2 Schematic of the data acquisition set-up for system response function and MTF measurements. . . . . . . . . . . . . 28

Figure 5.1 Step wedge 1 (soft tissue phantom) . . . . . . . . . . . . 37

Figure 5.2 Step wedge 2 (iodine phantom) . . . . . . . . . . . . . 37

Figure 5.3 Test phantom. . . . . . . . . . . . . . . . . . 44

Figure 6.1 Test phantom (mask). . . . . . . . . . . . . . . . 48

Figure 7.1 Log scale of relative flux as a function of focal spot size. . . . . . 50

Figure 7.2 Transfer response characteristics of CR 7400 . . . . . . . . . . . . 50

Figure 7.3 Extracted ROIs for MTF calculation. . . . . . . . . . . . . 51

Figure 7.4 ROI \#5 at 100-micron resolution: (a) ESF. (b) LSF. . . . . . . . 52

Figure 7.5 Measured MTFs and their corresponding fits. . . . . . . . . . . 53 
Figure 7.6 The difference in scan and subscan MTFs for (a) 50-micron and (b) 100-micron resolutions, displayed up to the Nyquist frequencies. . . . . . . . . . . . . . . 5 55

Figure 7.7 The 1D NPS in (a) horizontal, (b) vertical, and (c) diagonal directions. . . . . . . . . . . . . . . . . 56

Figure 7.8 The NEQ in (a) horizontal and (b) vertical directions. . . . . . 58

Figure 7.9 The DQE in (a) horizontal and (b) vertical directions. . . . . . . 59

Figure 7.10 High- and low-energy spectra, $a=1 \mathrm{~mm}^{2}$, (right y-axis) along with plots of logarithmic mass attenuation coefficients of Omnipaque-240 and CR plate (left y-axis). . . . . . . . . . 60

Figure 7.11 Calibration step wedges. . . . . . . . . . . . . . . . . . 62

Figure 7.12 Sampled calibration ROIs. . . . . . . . . . . . . . . . 62

Figure 7.13 Iodine calibration data. . . . . . . . . . . . . . . . 64

Figure 7.14 Plexiglas calibration data. . . . . . . . . . . . . . . . 65

Figure 7.15 The multi-well plexiglas plate as a part of the test phantom. . . . 66

Figure 7.16 (a) High- and (b) low-energy test images. . . . . . . . . . . . . 67

Figure 7.17 (a) Soft tissue-only and (b) iodine-only images. . . . . . . . . . 68

Figure 7.18 Measured and theoretical SNR and SdNR in the dual-energy and temporal subtracted iodine images, respectively. . . . . . . . . 69

Figure 7.19 (a) Mask and (b) post-contrast images. . . . . . . . . . . . . . 71

Figure 7.20 Temporal subtracted iodine image. . . . . . . . . . . . . 72 


\title{
List of Abbreviations
}

\author{
ADC Analog to Digital Converter \\ CCD Charge Coupled Device \\ CR Computed Radiography \\ CT Computed Tomography \\ DQE Detective Quantum Efficiency \\ DSA Digital Subtraction Angiography \\ ESF Edge Spread Function \\ FFT Fast Fourier Transform \\ FOV Field of View \\ HR High Resolution \\ HS High Speed \\ IP Imaging Plate \\ $\mathrm{keV} \quad$ Kilo Electron Volt \\ $\mathrm{kVp} \quad$ Kilo Voltage Peak \\ $\mathrm{kW} \quad$ Kilo Watt \\ LSF Line Spread Function \\ MTF Modulation Transfer Function \\ NEQ Noise Equivalent Quanta \\ NPS Noise Power Spectrum \\ PACS Picture Archiving and Communications System \\ PMT Photo Multiplier Tube \\ PSL Photostimulable Luminescence \\ PSP Photostimulable Phosphor Plate \\ QA Quality Assurance \\ QC Quality Control \\ ROI Region of Interest \\ RSD Relative Standard Deviation \\ SdNR Signal Difference to Noise Ratio \\ $\mathrm{S} / \mathrm{F} \quad$ Screen/Film \\ SNR Signal to Noise Ratio
}




\section{Chapter 1}

\section{Introduction}

The increasing use of genetic-manipulated small animals as models of human disease has spurred interest in new imaging methodologies. These models have become critical elements in the study of specific pathways for disease, potential therapies and safety of new pharmaceuticals.

Among small animals, mice and rats are commonly studied for such researches due to the large amount of established information on their genetic make-up, rapid rate of reproduction, and relatively low husbandry costs.

The extension of modern clinical imaging modalities to pre-clinical studies has revolutionized traditional imaging modalities, largely based on histological techniques, in which the studied animals are sacrificed and sliced. Such approaches are very invasive, labor intensive and do not permit longitudinal studies [9]. However, these problems can be overcome by extended in vivo imaging technologies, particularly micro-CT, micro-PET, and micro-MRI.

Tumor angiogenesis describes the development of new blood vessels within tumors. Angiogenesis is the result of genetic mutations within the tumor cells responsible for the release of angiogenic factors [28, 29]. "Zone of angiogenesis" is defined as the zone at the periphery of tumor where the greatest density of microvessels is found. Prior to the angiogenesis phase, tumors are small due to the limited access to circulating oxygen, nutrients and growth factors [49], and the tumor vessels are too small to be resolved using current small animal diagnostic imaging methods. However, these vessels appear to be abnormally "leaky", causing blood to pool around the tumor. The degree of angiogenesis varies with tumor types, with some tumors demonstrating greater neovascularisation than others [7]. Contrast agents, containing iodine-based non-ionic extracellular water-soluble compounds are used to increase the detectability of zone of angiogenesis, and thus providing a means for tracking tumor growth.

X-ray based micro-CT and micro-digital subtraction angiography (DSA) are important non-invasive imaging modalities to study tumorogenesis in small animals. The objective of this project is to evaluate the performance of a high-resolution computed radiography $(\mathrm{CR})$ system in detection of iodinated contrast agent in a small animal tumor model. Dual-energy and temporal subtraction techniques are implemented to enhance the contrast of iodinated regions and suppress soft tissue background. Wide dynamic range, fast throughput, low radiation level and cost are the potential ad- 
vantages of a CR system. This experiment can be considered as a fast pre-screening complementary procedure for small animal study by micro-CT when acquiring complete three-dimensional data is not necessary or time-efficient.

The framework of this thesis is prepared in five main chapters. "X-ray Imaging Background" introduces the fundamentals and mechanisms of X-ray production and $\mathrm{X}$-ray interactions with matter relevant to X-ray diagnostic radiology. The structure of a computed radiography workflow is discussed in "Computed Radiography $(\mathrm{CR})$ ", including the history, physics and imaging performance in general, quality improvements and new developments. "Image Quality Assessment of a Computed Radiography System" discusses the imaging performance characteristics of a Kodak high-resolution desktop-sized CR (CR 7400, Kodak Dental Systems, Carestream Health, Rochester, New York) used in the project. "Dual-energy Subtraction Technique" details the history and theories of dual-energy subtraction and dual-energy decomposition techniques, algorithms, designs of calibration and test phantoms, and computer modeling. Similarly, "Temporal Subtraction Technique" encompasses the history, underlying physics of the technique, phantom design and computer modeling. 


\section{Chapter 2}

\section{X-ray Imaging Background}

\subsection{X-ray Production}

\subsubsection{Fundamentals and Mechanisms}

$\mathrm{X}$-ray is a form of electromagnetic radiation with the wavelength ranging from a few picometers to a few nanometers. The energy of each X-ray photon is proportional to its frequency of $\nu$, with the proportionality constant given by Plank's constant, $h$, as described by [38]

$$
E=h \nu=\frac{h c}{\lambda}
$$

where $h=6.63 \times 10^{-34}$ J.s, $c$ is the speed of light $\left(3 \times 10^{8} \mathrm{~m} / \mathrm{s}\right)$, and $\lambda$ is the wavelength of the X-ray photon measured in $n m$. The X-ray energy is usually expressed in the units of $\mathrm{eV} .1 \mathrm{eV}=1.602 \times 10^{-19} \mathrm{~J}$; the amount of kinetic energy gained by a single unbound electron when it accelerates through an electric potential difference of one volt. The most common X-ray production technology in the majority of radiology departments is the standard X-ray tube. Figure 2.1 shows a schematic of an X-ray tube. The flow of current through a filament in the cathode causes electrons to boil off (Thermionic Emission). A high electrical potential difference, typically between 80 to $150 \mathrm{kVs}$, between cathode and anode pulls the electrons towards the anode at a very high speed in the vacuum tube. The electrons interact with the anode atoms and produce X-rays. Since the kinetic energy of electrons is converted into electromagnetic radiation, the maximum possible energy of produced X-rays equals the entire kinetic energy of the electrons and Equation 2.1 can be rewritten as [38]

$$
E=\frac{1.24 \times 10^{3} \mathrm{eV} . \mathrm{nm}}{\lambda}
$$

The energy of diagnostic X-rays varies roughly from $10 \mathrm{keV}$ to $150 \mathrm{keV}$. Although X-rays with much higher energy have better penetrating power, they provide little low-contrast information and are of little interest for diagnostic imaging. On the other hand, X-rays with energies in the range of $100 \mathrm{eV}$ to $10 \mathrm{keV}$ hardly penetrate thicker layers of materials. They are classified as "soft" X-rays and have no value in the diagnostic radiology.

The majority of high-speed electron interactions with an anode involve energy transfers to the electrons of anode atoms which knock them out of the atom and 


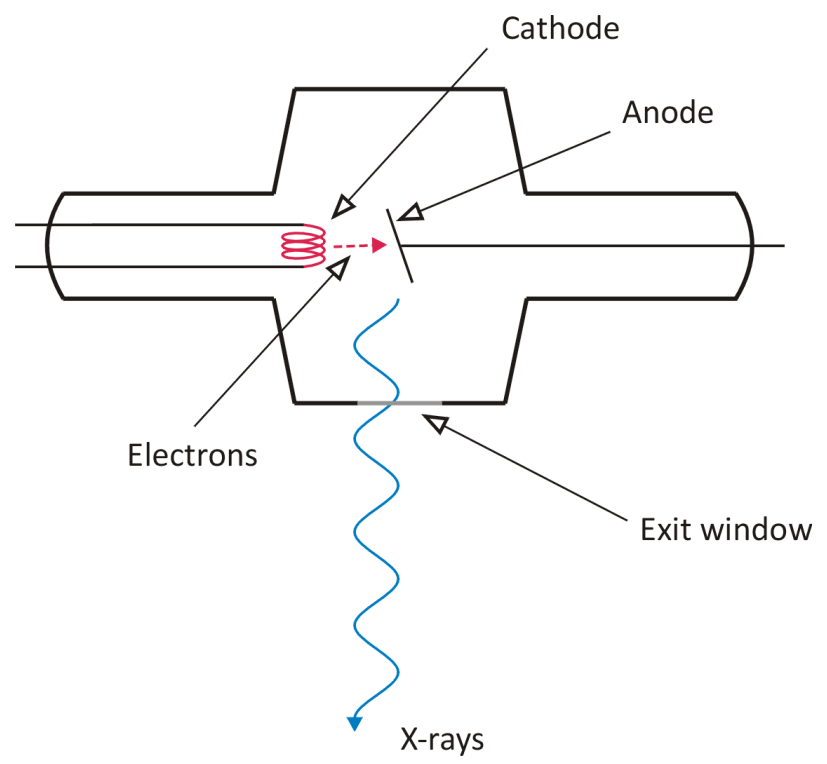

Figure 2.1: X-ray tube schematic.

produce ionized anode atoms. This type of collision does not generate X-rays and gives rise to the production of heat in the anode. In a typical X-ray tube, over $99 \%$ of the input energy is dissipated as heat [21]. Brehmsstrahlung and characteristic radiations are electron to atom interactions in which diagnostic X-rays are generated.

\subsubsection{Brehmsstrahlung Radiation}

Brehmsstrahlung radiation is the main source of X-rays produced by diagnostic $\mathrm{X}$-ray tubes. The first type of brehmsstrahlung radiation happens when high-speed electrons are deflected by the coulomb field of the nucleus of the anode atoms. The sudden deceleration of electrons involves loss of kinetic energy (velocity). This energy loss is promptly emitted as X-rays [38]. The energy of resulting X-rays depends on the amount of incident kinetic energy given off during interaction. As the amount of interaction increases (i.e.: the passing electrons are closer to the nucleus), the energy of generated X-rays also increases (Figure 2.2).

The total intensity of brehmsstrahlung radiation $\left(I_{b}\right)$ resulting from a charged particle of mass $m$ and charge ze incident onto target nuclei with charge $Z e$ is proportional to [12]

$$
I_{b} \propto \frac{Z^{2} z^{4} e^{6}}{m^{2}} .
$$

This equation states that production of brehmsstrahlung radiation is significantly (over 3 million times) less efficient when a massive particle such as an alpha particle replaces an electron [12]. Therefore, high-speed electrons become the practical choice for producing brehmsstrahlung. Moreover, the brehmsstrahlung production 


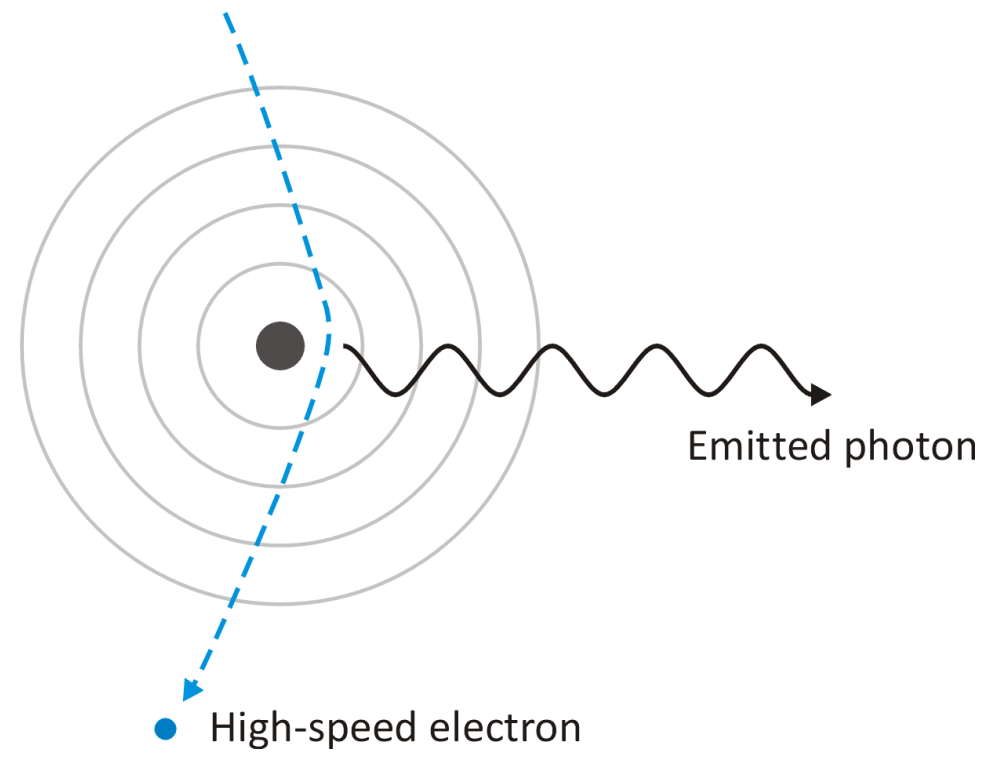

Figure 2.2: Brehmsstrahlung.

increases rapidly when a material with high atomic number is used to construct the anode.

The second type of brehmsstrahlung radiation involves direct collision of highspeed electrons with the nucleus of anode atoms in which the entire electron kinetic energy appears as brehmsstrahlung. The X-rays produced by this interaction have the maximum possible energy and represent the upper energy limit in the X-ray spectrum. But the probability of such interaction is low [38].

\subsubsection{Characteristic Radiation}

In the production of characteristic X-rays, an accelerated electron strikes one of the inner-shell electrons of an atom with energy greater than that electron's binding energy and ejects the orbital electron, creating a vacancy. Both striking and orbital electrons will then go on to interact further with other target atoms, until their kinetic energy is spent. The hole is filled by an outer-shell electron, and according to the energy conservation law, the energy of the released photon is equal to the energy difference between the binding energies of two shells. Thus, the ejection of a K-shell electron sets up a cascade of electron transitions, until vacancies in the outermost shells are filled by free electrons in the environment (e.g., bombarding and ejected electrons) [12]. For example, in Figure 2.3, an 80-keV electron impinges on a tungsten K-shell electron. The incoming electron has more energy than the binding energy of tungsten K-shell electron $(70 \mathrm{keV})$, liberating the K-shell electron and an outer-shell electron fills in the void. If the replacement electron is from the $\mathrm{L}$ shell $(11 \mathrm{keV})$, a $59-\mathrm{keV}$ photon is emitted. Similarly, when a M-shell (3 keV) electron moves to the 

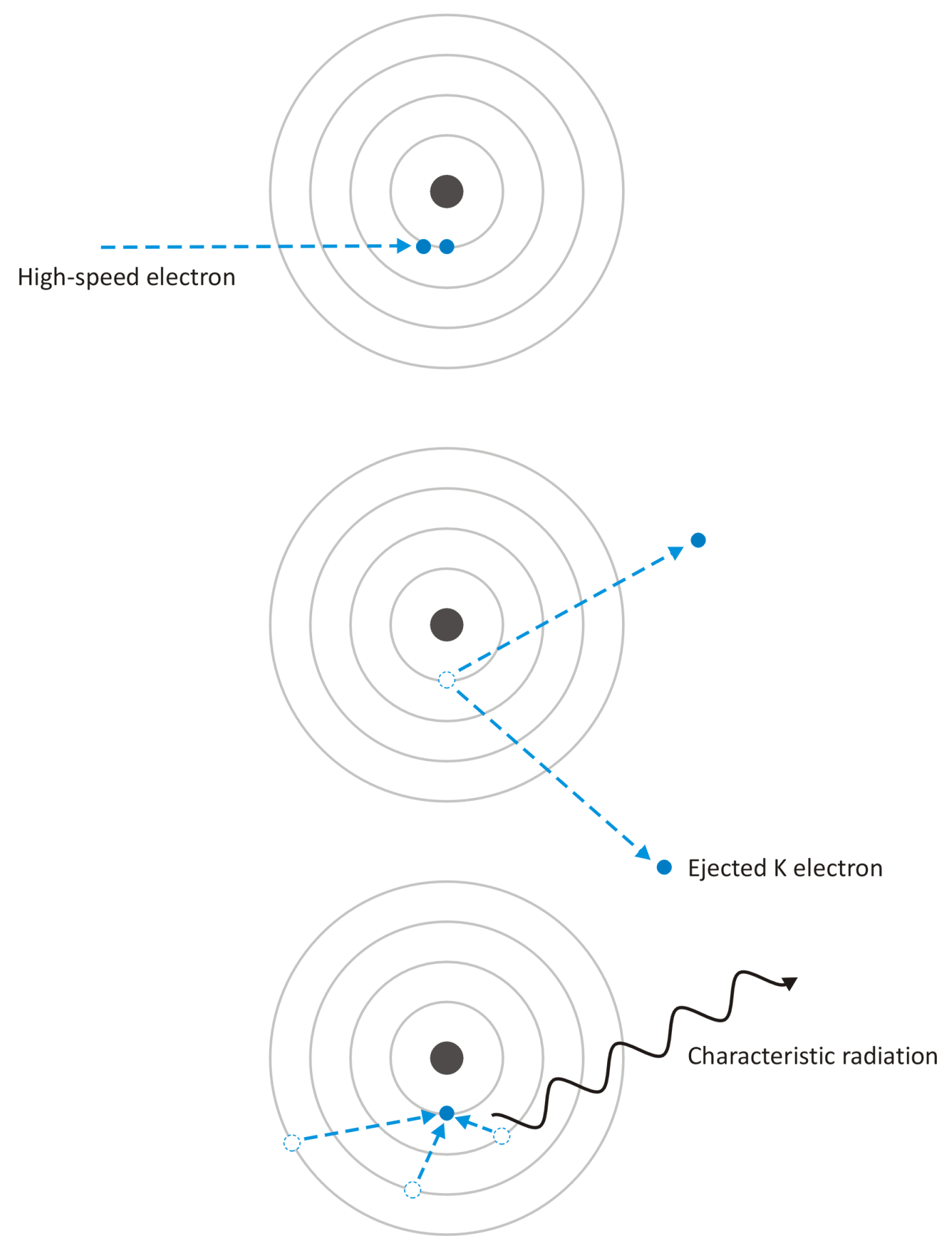

Figure 2.3: Characteristic X-ray production. 
K-shell, the released X-ray photon has $67 \mathrm{keV}$ of energy. This high-energy photon can then interact with other atoms or it can escape from the anode as a characteristic $\mathrm{X}$-ray. A characteristic X-ray released during transition of an electron between adjacent shells is known as an $\alpha \mathrm{X}$-ray. If transition is among nonadjacent shells, a $\beta$ $\mathrm{X}$-ray is produced. For example, a $\mathrm{K}_{\alpha}$ emission happens when $\mathrm{K}$ shell receives an electron donated by the next shell up (the $\mathrm{L}$ shell). A $\mathrm{K}_{\beta}$ X-ray reflects a transition of an electron from the $\mathrm{M}$ shell to the $\mathrm{K}$ shell. Note that each element in the periodic table has its own unique atomic shell binding energies, thus the energies of characteristic X-rays are unique to each atom.

\subsection{X-ray Interactions with Matter}

Generally, there are five types of interactions with matter by photons in radiological physics:

1- Photoelectric effect

2- Compton scattering

3- Rayleigh (coherent) scattering

4- Pair production

5- Nuclear photoeffect

For the production of an electron-positron pair, a photon energy of at least 1.02 $\mathrm{MeV}$ is needed and photonuclear reactions are only significant for photon energies above a few million electron volts $(>2.04 \mathrm{MeV})$ [25]. Therefore, only the first three processes are relevant to X-ray diagnostic radiology (10 keV through $150 \mathrm{keV}$ ). The photoelectric effect and Compton scattering result in transfer of energy to electrons, which is then imparted to the material along their tracks.

\subsubsection{Photoelectric Effect}

In photoelectric interaction (Figure 2.4), an X-ray photon with energy greater than the binding energy of an inner-shell electron knocks out the electron. Part of the photon energy is used to overcome the binding energy of the electron and the remainder is given to the electron as kinetic energy. The free electron is often called photoelectron. When an electron from outer shells fills in the void, a photon having the characteristic radiation of material is emitted. Thus, the photoelectric effect produces a positive ion, a photoelectron, and a photon of characteristic radiation [38]. This phenomenon was discovered by Albert Einstein in 1905, for which he received the Nobel Prize in Physics in 1921.

While the mechanism of characteristic radiation production in a photoelectric process is similar to the one produced as part of X-ray spectrum in high-speed elec- 


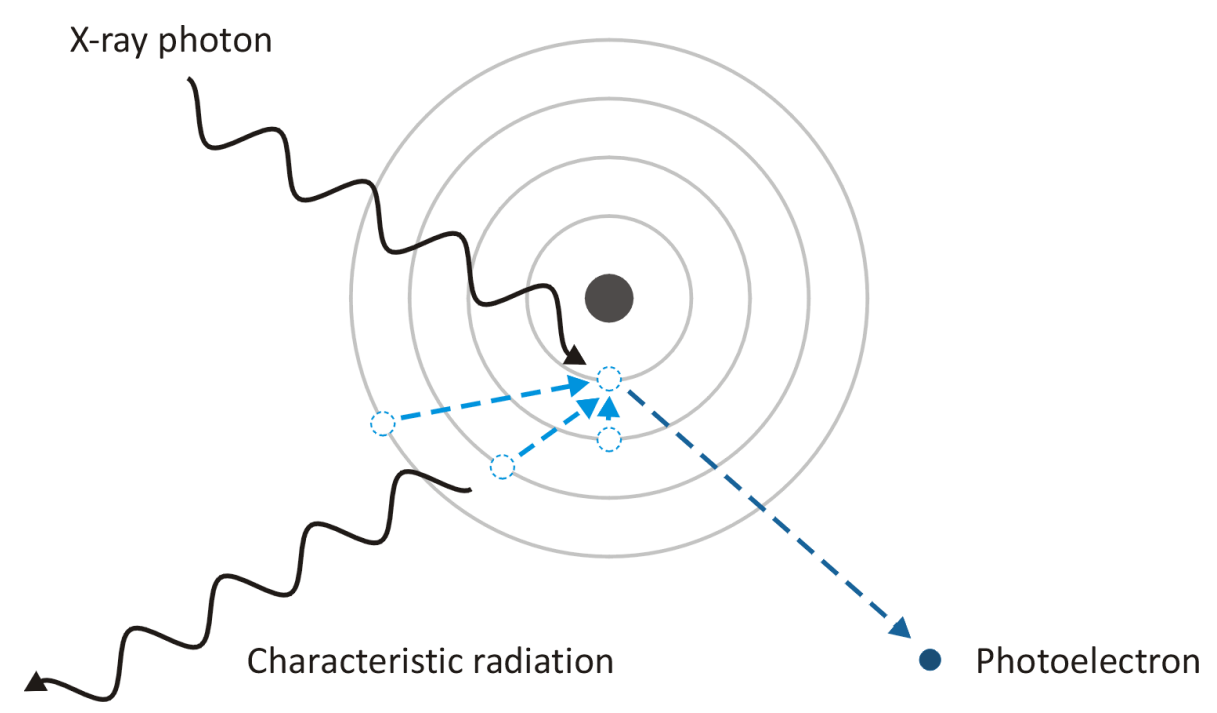

Figure 2.4: Photoelectric effect.

trons/anode electrons interactions, some practical differences should be mentioned. As already discussed, X-rays are produced by high-speed electrons bombarding the anode made of a material with high-atomic number, usually tungsten $(\mathrm{Z}=74)$, which result in characteristic X-rays $\left(\mathrm{K}_{\alpha}, \mathrm{K}_{\beta}\right.$, etc.) with quite high energies. However, $\mathrm{X}$-rays used in medical imaging interact first with the patient and then with the detector. Soft tissue-like materials are mainly composed of low-atomic number elements such as hydrogen $(Z=1)$, carbon $(Z=6)$, nitrogen $(Z=7)$, and oxygen $(Z=8)$, all with the K-shell binding energies of roughly $500 \mathrm{eV}$ or less. Even, calcium $(Z=20$, a constituent of bone) has K-shell binding energy of $4 \mathrm{keV}$ [12]. Therefore, X-rays produced in such low-energy interactions do not travel far before being attenuated. Since the mean free path of $1-\mathrm{keV}$ X-ray photon in muscle tissue is about $2.7 \mu \mathrm{m}$ (less than the dimensions of a typical human cell) [12], we can safely assume that all characteristic X-rays produced in soft tissue by photoelectric effect are re-absorbed locally in adjacent tissue.

Although the photoelectric interaction can happen with electrons in any shell, it is most probable to occur with the most tightly bound electron the photon is able to dislodge (i.e. the K shell). Since the process is again concerned with bound electrons, it is favored in materials of high atomic number $(Z)$. In addition, it is also favored by low photon energies $(E)$, as shown by [38]

$$
P_{\text {p.e. }} \propto \frac{Z^{3}}{E^{3}} .
$$

Equation 2.4 indicates that at energies where the photoelectric effect dominates, e.g. mammography, tissues with close atomic numbers may result in a substantial difference in the probabilities of photoelectric effects, which in turn, leads to a significant difference in absorption of X-ray photons. This will then result in a significant 
contrast between different tissues. In addition, it implies that low-energy X-rays are the main contributors to the low-contrast differentiation in tissues.

\subsubsection{Compton Scattering}

The most important effect in radiology involving unbound electrons is inelastic scattering or the Compton effect, named after Arthur Holly Compton who received the Noble Prize in physics in 1927. Compton scattering typically occurs at higher $\mathrm{X}$-ray energies where the energy of X-ray photon is much higher than the binding energy of orbital electron. An incident X-ray photon makes a billiard ball-type collision with an outer-shell (essentially free) electron, freeing it from the atom, causing ionization. The incident X-ray photon is then deflected or scattered with partial loss of its initial energy [12]. In other words, energy deposition happens such that the photon increases its wavelength and changes direction while the atomic electron is knocked out of its valence shell. The scattered photon and electron may then undergo further collisions before exiting the patient. Thus, a Compton interaction produces a positive ion, a "recoil" electron, and a scattered photon (Figure 2.5).

Equation 2.5 is used most frequently to describe the Compton process [25]:

$$
\lambda^{\prime}-\lambda=\frac{h c}{m_{0} c^{2}}(1-\cos \theta)
$$

where $\lambda^{\prime}$ and $\lambda$ are the wavelengths of scattered and incident photons, respectively. $\theta$ is the scattering angle. $h$, Planck's constant is $6.62 \times 10^{-34} \mathrm{~J} . s$, and $m_{0} c^{2}$ $(=511 \mathrm{keV})$ is the rest mass of the valence electron. This equation shows that the change in wavelength $(\Delta \lambda)$ when the photon is scattered through an angle $\theta$, is independent of photon energy. However, assuming the photon loses little energy and the energy of the scattered photon is approximately the same as the incident photon, it can be shown that the change in energy of photon $\Delta E$ is given by [25]

$$
\Delta E=\frac{E^{2}}{m_{0} c^{2}}(1-\cos \theta) .
$$

Thus, the scattered photon energy loss does depend on the incident photon energy and scattering angle. For example, when a photon is scattered at $60^{\circ}$, the proportion of energy taken by the electron varies from about $2 \%$ at $20 \mathrm{keV}$ to $8 \%$ at $80 \mathrm{keV}$ and $14 \%$ at $140 \mathrm{keV}$.

Note that low-energy X-ray photons are preferentially backscattered $\left(\theta>90^{\circ}\right)$, whereas high-energy photons are more probable for forward scattering $\left(\theta<90^{\circ}\right)$ [38]. Since a small portion of the incident photon energy is absorbed in a Compton interaction, the patient absorbed dose is considerably less than the photoelectric interaction.

Unlike the photoelectric effect, the probability of a Compton scattering is solely proportional to the electron density of the material (over the diagnostic X-ray energy 


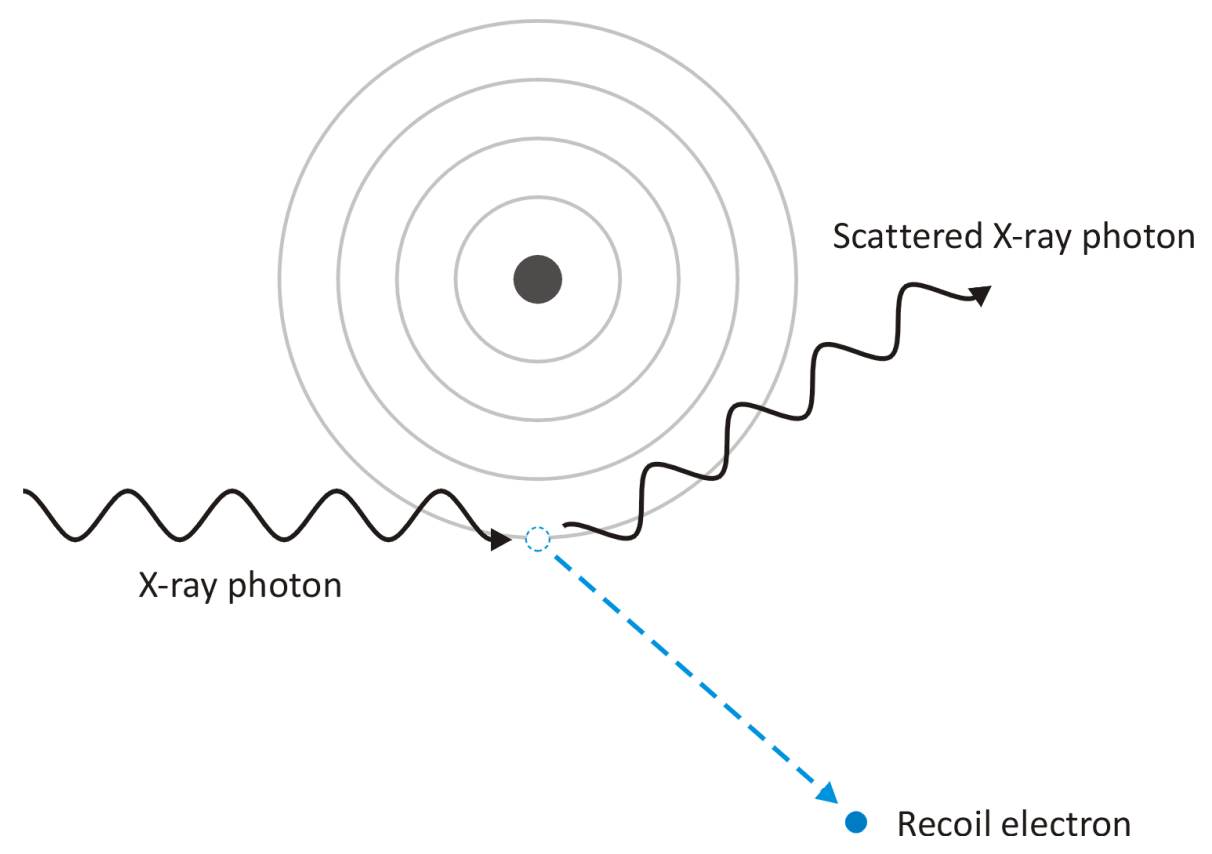

Figure 2.5: Compton scattering.

region from 10 to $100 \mathrm{keV}$. For $\left.\mathrm{E}>100 \mathrm{keV}, P_{c . s .} \propto \frac{1}{E}[12]\right)$. Since various types of soft tissues have almost similar densities, a Compton interaction provides little contrast differentiation between soft tissues.

\subsubsection{Rayleigh (Coherent or Thomson) Scattering}

In Rayleigh scattering, the incident X-ray photon is scattered as a result of interaction with the electric field of an orbiting electron. Since the energy of scattered photon remains equal to the energy of incident photon and no energy is converted to the kinetic energy, the ionization does not occur. However, the scattered X-ray experiences a change in its trajectory relative to incident X-ray [12]. Rayleigh scattering is most likely to happen in the forward direction for low-energy X-rays and absorbers with high atomic numbers, which produces a slightly broadened X-ray beam, undesirable in medical imaging.

Although the Rayleigh scattering is likely to happen at all X-ray energies, as stated by Equation 2.7, it never accounts for more than $10 \%$ of the total interaction processes in diagnostic radiology [38].

$$
P_{r . s .} \propto \frac{Z^{2}}{E} .
$$

As previously discussed, the photoelectric and Compton scattering are the main interactions over the range of X-ray energies in diagnostic radiology. The photoelectric 
effect is very dependent on the atomic number; the Compton effect is not. When the energy of X-rays increases, the percentage of Compton interactions also increases, whereas the probability of photoelectric effect decreases rapidly. Moreover, more energy is transferred in a photoelectric interaction than in a Compton scattering.

Understanding the difference in the relative importance of these two effects at different energies is quite fundamental to appreciating the origin of radiological image contrast based on differences in the atomic number and applied energy. Generally in the context of energy transfer percentage, photoelectric effect and Compton scattering are the dominant interactions for the energies up to $50 \mathrm{keV}$ and 90 through $150 \mathrm{keV}$, respectively. For the energies between 50 and $90 \mathrm{keV}$, both interactions are important [38].

\subsection{Attenuation Coefficients}

When a parallel beam of a number $N_{0}$ of mono-energetic photons passes through a flat plate of material of thickness $t$, the number of transmitted photons, $N$, is given by [25]

$$
N=N_{0} e^{-\mu t}
$$

where $\mu$ is the linear attenuation coefficient of the material. Equation 2.8 is generally called the exponential law of attenuation; the Lambert-Beers law. It also holds for the intensity of the photon beam. Since $\mu$ is dependent on the material density, the quantity usually tabulated is the mass attenuation coefficient, $\frac{\mu}{\rho}$, in which the dependence on the density has been removed.

Photoelectric $\left(\frac{\tau}{\rho}\right)$, Compton $\left(\frac{\sigma}{\rho}\right)$, and Rayleigh $\left(\frac{\sigma_{r}}{\rho}\right)$ interactions occur independently of each other and their effects can be combined as shown by [12]

$$
I=I_{0} e^{-\left(\frac{\tau}{\rho}\right) \rho t-\left(\frac{\sigma}{\rho}\right) \rho t-\left(\frac{\sigma_{r}}{\rho}\right) \rho t-\ldots},
$$

or

$$
I=I_{0} e^{-\left(\frac{\tau}{\rho}+\frac{\sigma}{\rho}+\frac{\sigma_{r}}{\rho}+\ldots\right) \rho t},
$$

leading to a simple relationship

$$
\frac{\mu}{\rho}=\left(\frac{\tau}{\rho}\right)+\left(\frac{\sigma}{\rho}\right)+\left(\frac{\sigma_{r}}{\rho}\right)+\ldots
$$

where additional interaction coefficients can be added if they contribute significantly to the value of $\frac{\mu}{\rho}$.

Equation 2.10 indicates that the total mass attenuation coefficient of a material through which X-ray photons propagate, is equal to the sum of all the components mass attenuation coefficients obtained by considering each process independently.

Within the range of energies used in small animal imaging $(\sim 80 \mathrm{kVp})$, the dominant X-ray cross sections in absorption are Compton scattering and photoelectric 
effect. Figure 2.6 shows the absorption cross sections (Compton scattering, photoelectric effect, Rayleigh scattering and their totals) of soft tissue, iodine (iodinated contrast agent), and barium (main constituent of CR imaging plate). Note how the photoelectric effect dominates the X-ray absorption cross sections for iodine and barium due to their high atomic numbers $\left(\mathrm{Z}_{\mathrm{I}}=53, \mathrm{Z}_{\mathrm{Ba}}=56\right)$, while Compton scattering is the dominant interaction for soft tissue $\left(Z_{\text {Tissue }} \approx 7\right)$. The $\mathrm{X}$-ray cross sections and the materials involved (tissue, iodine, and barium) form the basis for the dual-energy subtraction technique discussed later in Chapter 5 .

\subsection{X-ray Detection}

A significant leap in X-ray detection from film to digital detectors has allowed acquisition of images with higher spatial and contrast resolution and introduction of computed tomography (CT) [71]. Image quality in medical radiology is intimately linked to the performance of the X-ray detectors. Yaffe et al. [71] have reviewed the underlying physics of digital X-ray detectors and discussed key parameters such as spatial resolution, uniformity of response, contrast sensitivity, dynamic range, acquisition speed, and frame rate.

Photostimulable phosphor (PSP) based computed radiography (CR) has gained an increasing widespread acceptance since it was first commercialized in 1983 [14]. Numerous publications have shown CR to be an acceptable and sometimes more favorable choice over conventional screen/film systems for a variety of applications $[68,6,47]$. The structure of a computed radiography workflow is discussed in the next chapter. In a nutshell, advantages with $\mathrm{CR}$ compared to film/screen system include: convenient storage of radiological data, increased flexibility in image processing, and consistent reproducibility along with a greater dynamic range, wider exposure latitude, and reduced patient exposure. 

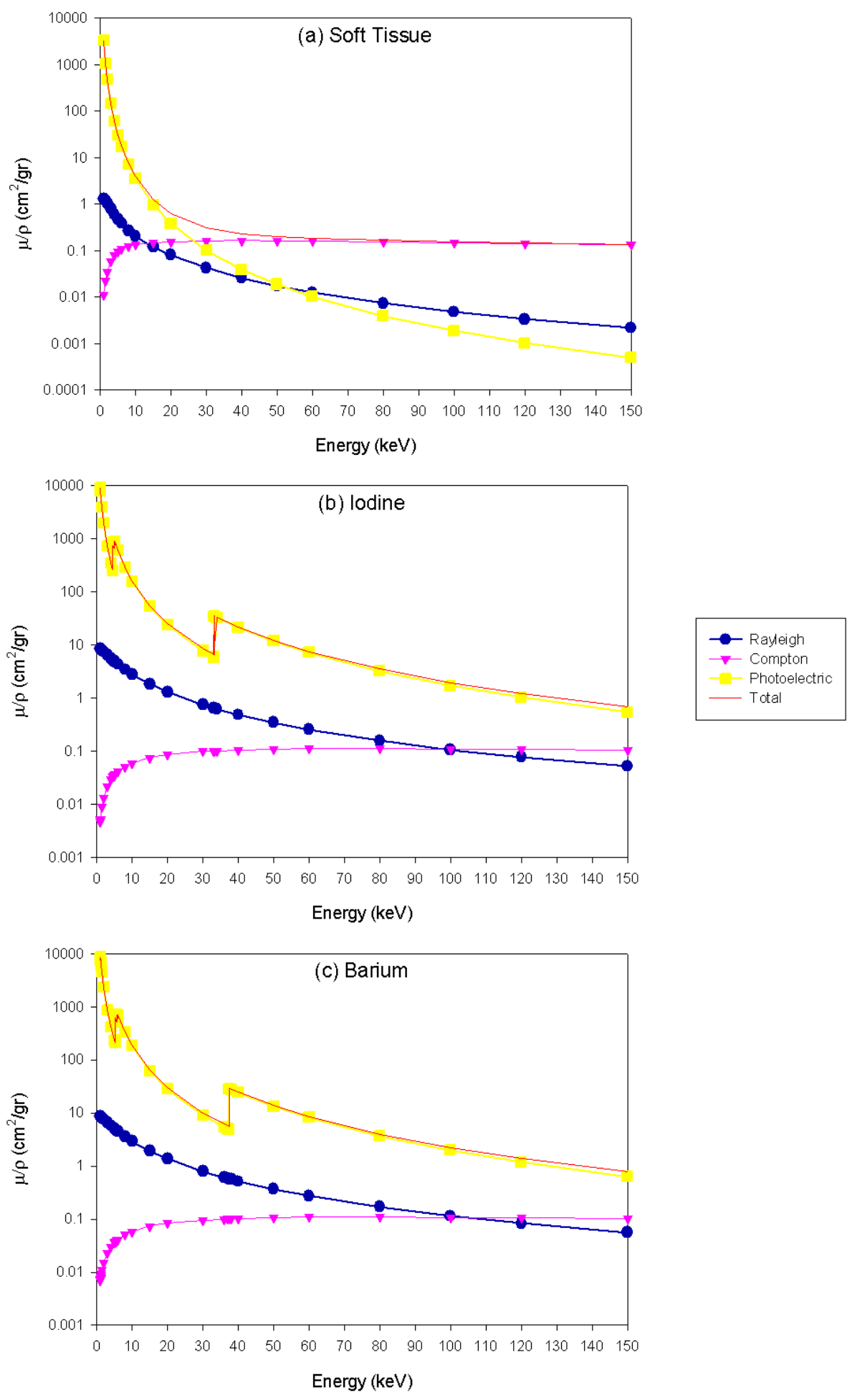

Figure 2.6: Log-scale plots of mass attenuation coefficients contributed by the three main interactions for soft tissue (a), iodine (b), and barium (c). 


\section{Chapter 3}

\section{Computed Radiography (CR)}

\subsection{Introduction}

Computed radiography (CR) technology has nearly evolved three decades of clinical use, beginning with the introduction of Fuji FCR 101 in 1983. Since then and particularly in the 1990s, several manufacturers have realized the opportunities and importance of CR clinical acquisition systems as necessary to the implementation of Picture Archiving and Communications Systems (PACS). These manufacturers have provided a wide range of capabilities including high-throughput, multi-plate stackers, cassetteless, high-speed automated CR acquisition devices, and compact desktopsized single-plate readers to address the needs of the largest hospitals to the smallest outpatient clinics $[63,56]$.

The steps involved in creating a CR image are not so different from a screen-film system $(\mathrm{S} / \mathrm{F})$. But $\mathrm{CR}$ replaces the film with a re-usable imaging plate and inserts a digital computer between the image detector and display to perform a number of image processing tasks, including compensating for exposure errors, applying appropriate contrast characteristics, enhancing image details, and storing and distributing image information in digital form [72].

\subsection{CR versus Screen/Film}

\subsubsection{Image Formation}

In a conventional analog $\mathrm{S} / \mathrm{F}$ system, a luminescent intensifying screen absorbs $\mathrm{X}$-rays and promptly emits light, exposing the film. Chemical development of the latent image in the film produces more optical density (blackness) in the highly-exposed areas and less blackness in the areas with poor light exposure. In a $\mathrm{S} / \mathrm{F}$ system, the film is the acquisition, display, and storage medium, requiring a number of design trade-offs such as X-ray exposure latitude versus display contrast, and sensitivity versus noise. This is the primary reason why there are different types of $\mathrm{S} / \mathrm{F}$ systems for various clinical applications . 
$\mathrm{CR}$ is like an electronic-age version of projection X-ray imaging with film without fluorescent screens [62]. A photostimulable phosphor (PSP) plate is usually held within a light-tight cassette, serving only as a protective housing with no fluorescence characteristics. The plate, typically made of an europium-doped barium-fluorohalide crystallized matrix, absorbs X-ray photons and some electrons from dopant ions are elevated and trapped into a meta-stable state, just below the conduction band (latent image). The latent image is then "read" by a red or near-infrared laser, exciting the trapped electrons into the conduction band, from which they return to their original ground state with the release of energy in the form of green, blue, or ultraviolet light - the photostimulable luminescence (PSL) phenomenon (Figure 3.1). The intensity of emitted light is proportional to the amount of X-rays absorbed by the storage phosphor [62].

Figure 3.2 shows the stimulation curve of a typical storage phosphor. The amount of stored latent signal extracted from the screen depends on the total amount of energy deposited by the stimulation source which, in turn, depends on the intensity of the stimulating light and the length of time a given screen area is stimulated, which is also called the dwell time. However, this relationship is nonlinear. As the stored latent image becomes depleted, it becomes harder to extract the remaining signal, even with large amount of deposited stimulation energy [62].

Early CRs used a helium-neon laser $\left(\lambda_{s} \approx 633 \mathrm{~nm}\right)$ to stimulate the trapped electrons while most modern $\mathrm{CR}$ systems use more compact, efficient and long-lasting red-emitting laser diodes $\left(\lambda_{s} \approx 670-690 \mathrm{~nm}\right)$ as the light source. Red wavelengths are well matched to the stimulation spectrum of commonly used barium fluorohalide plates and are different enough from the blue or green emitted wavelengths $\left(\lambda_{e} \approx 400-500 \mathrm{~nm}\right)$ that they do not interfere with detection. The separation of emitted light wavelengths from stimulating wavelength is crucial to the function of $\mathrm{CR}$ (Figure 3.3) [62, 56].

It is interesting to note that PSP plates also produce light upon exposing to $\mathrm{X}$-rays, similar to conventional intensifying screens. But this prompt emission is wasted and has no role in formation of the clinical image. Unfortunately, even in modern CRs, about half of the potential stored signal is lost to prompt emission during X-ray exposure.

In the conventional CR scanners, the readout process is done by a precision laser spot-scanning mechanism in which the optical beam traverses the imaging plate (IP) surface in a raster pattern. The stimulated light emitted from the IP is collected with optics coupled to a photomultiplier tube (PMT), the analog output of which is amplified, sampled (once for each pixel), and sent through an ADC. Finally, the pixel's signal ends up with a value between 0 and 1023 for a 10-bit ADC, or between 0 and 4095 for a 12-bit ADC, appropriate for local display or transmission to the PACS (Figure 3.4) [72].

Not all of the stored energy in the PSP is released during the readout process. To erase any residual latent image and prepare the IP for a new X-ray exposure, it 

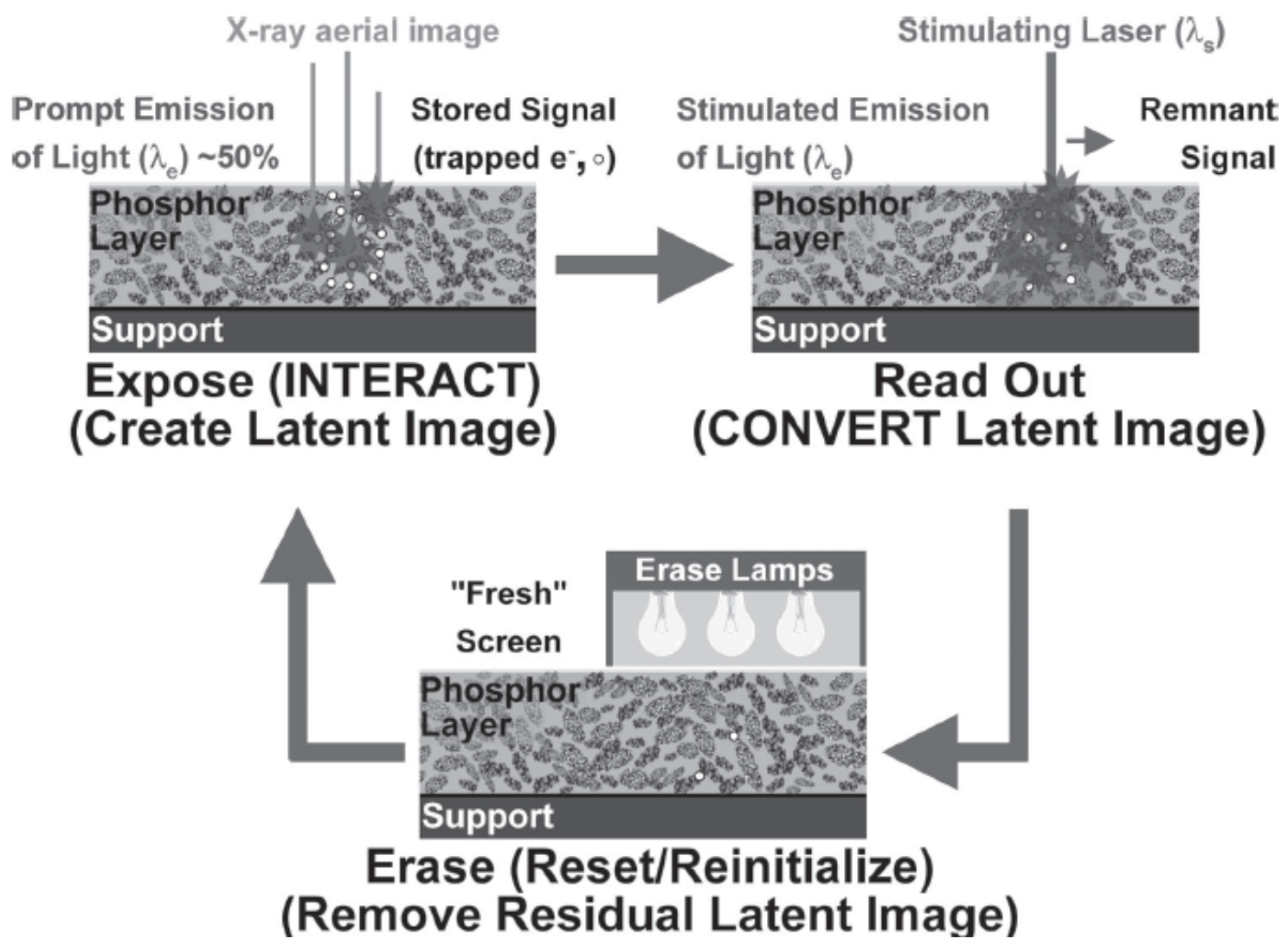

Figure 3.1: CR three-step imaging cycle: expose, readout, and erase.

Source: Reprinted with permission from Radiological Society of North America (RSNA). R. Schaetzing, "Computed radiography technology," in Advances in digital radiography: categorical course in diagnostic radiology physics, E. Samei and M. Flynn, Eds., Radiological Society of North America, 2003. 


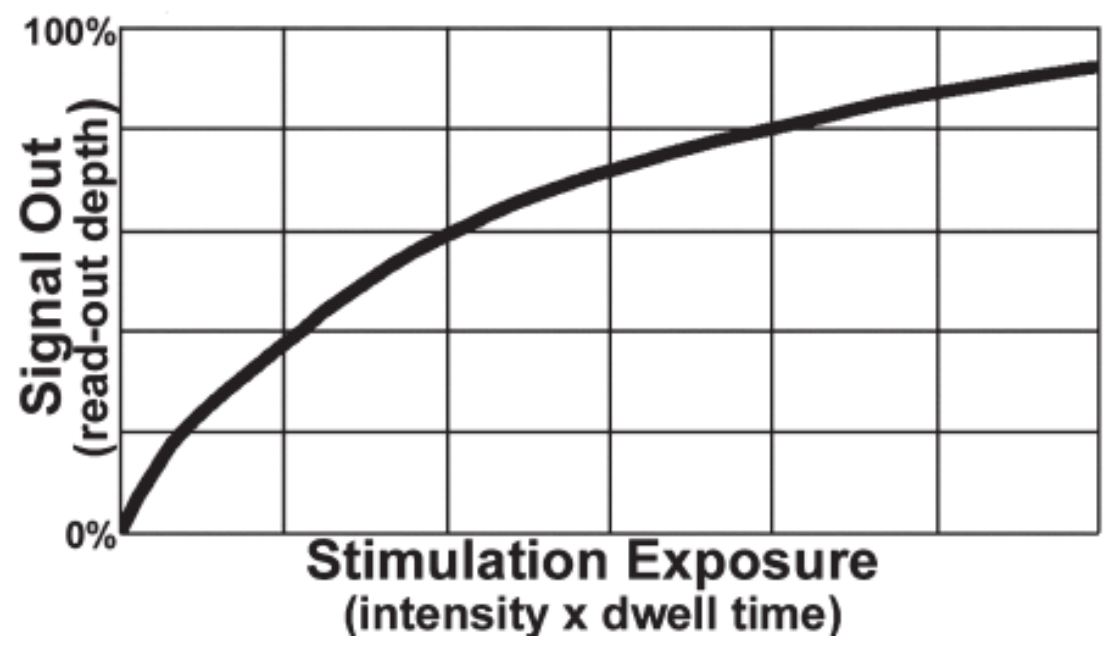

Figure 3.2: Storage phosphor stimulation curve.

Source: Reprinted with permission from Radiological Society of North America (RSNA). R. Schaetzing, "Computed radiography technology," in Advances in digital radiography: categorical course in diagnostic radiology physics, E. Samei and M. Flynn, Eds., Radiological Society of North America, 2003.

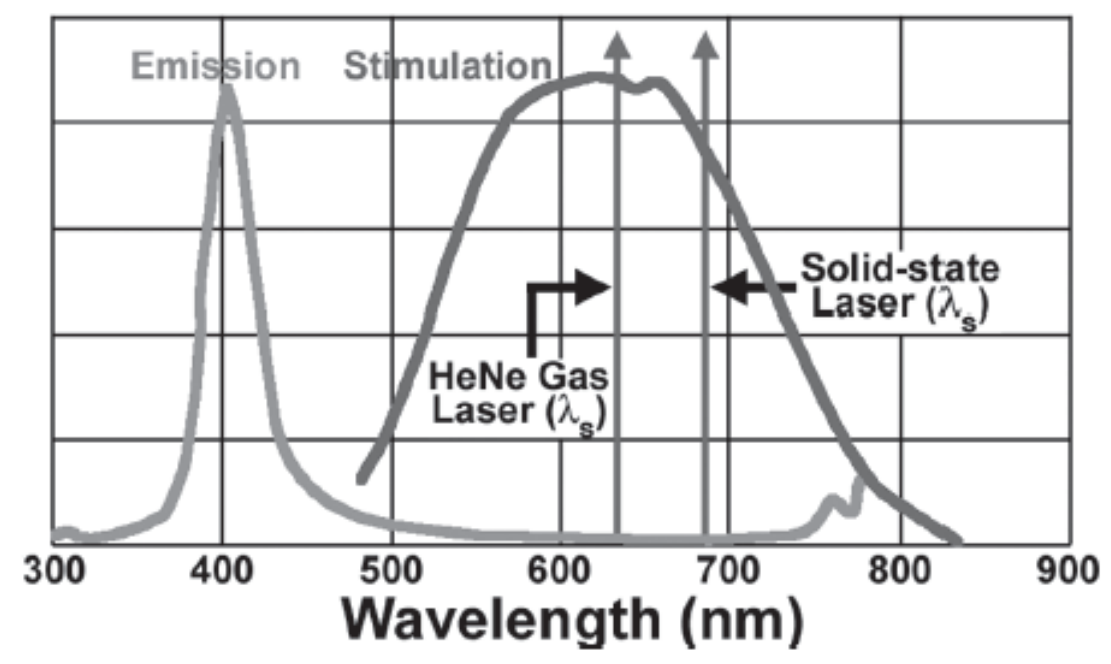

Figure 3.3: Storage phosphor emission and stimulation spectra.

Source: Reprinted with permission from Radiological Society of North America (RSNA). R. Schaetzing, "Computed radiography technology," in Advances in digital radiography: categorical course in diagnostic radiology physics, E. Samei and M. Flynn, Eds., Radiological Society of North America, 2003. 


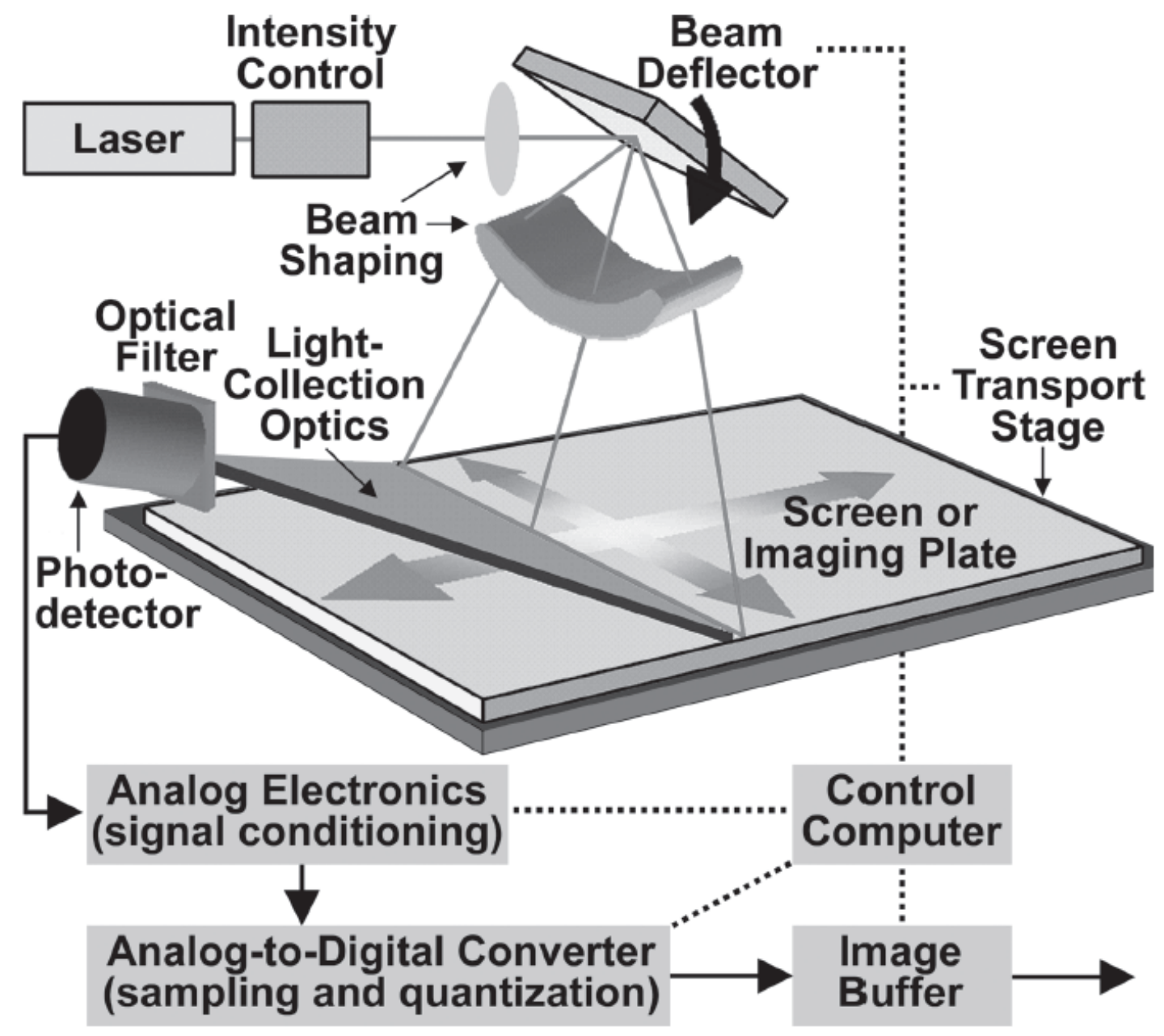

Figure 3.4: Main components of a flying-spot CR scanner.

Source: Reprinted with permission from Radiological Society of North America (RSNA). R. Schaetzing, "Computed radiography technology," in Advances in digital radiography: categorical course in diagnostic radiology physics, E. Samei and M. Flynn, Eds., Radiological Society of North America, 2003. 
is necessary to "flood" the IP with high-intensity light (typically fluorescent), thereby releasing the majority of remaining trapped electrons. Note that the erasure step is not $100 \%$ effective; that is, the stored signal can never be erased completely. However, as long as the highest leftover stored signal on the plate after erasure remains well below the lowest expected signal from the next exposure, this residual "contamination" is unimportant [72].

\subsubsection{System Characteristics}

There are important differences between S/F and CR technologies. Probably, the two biggest are exposure latitude and system response linearity. Film latitude is usually limited to a fairly narrow exposure range of about 30-40:1 by photon detection threshold and signal saturation effects ("toe" and "shoulder" of the film characteristic response curve). By comparison, the exposure limits of CR are about 10000:1. This wide exposure latitude and the effective linear detector characteristic curve allow a wider range of exposure information to be captured. In addition, the wider latitude allows it to be used under a broad range of exposure conditions without need of adjusting or changing the detector, which significantly reduces the number of retakes. However, this is a two-edged sword, since it can mask systemic problems related to equipment malfunction or poor radiographic technique (e.g., too high a dose) that would be immediately obvious with $\mathrm{S} / \mathrm{F}$ systems. CR systems usually contain special software to help users detect and monitor such problems [72, 63].

As a result of CR's convenient workflow, dataflow, portability, wide dynamic range and exposure "forgiveness", it can either be placed in a central location, similar to the centralized high-volume chemical processors in a typical $\mathrm{S} / \mathrm{F}$ environment, or closer to where the images are acquired even in difficult imaging situations, such as in an emergency room, examination room, intensive care unit or a trauma center $[62,72]$.

\subsection{Imaging Performance of CR}

Many imaging performance metrics can be measured in CR, including signal response (input/output [I/O] relationship), spatial resolution (typically expressed as Modulation Transfer Function [MTF]), noise (typically expressed as Noise Power, or Weiner Spectrum), and dose efficiency (represented by the Detective Quantum Efficiency [DQE]). Since the detailed imaging performance of CR is discussed in the next chapter, "Image Quality Assessment of a Computed Radiography System", general performance issues related to input/out relationship and the factors affecting spatial resolution and noise are discussed here. 


\subsubsection{Input/Output Relationship}

Storage phosphor plates are linear detectors over four or five orders of exposure magnitude. However, this wide dynamic range can be somewhat limited by the performance of the scanner. For example, high X-ray exposures can produce light signals high enough to saturate PMTs. At the low-signal end, the scattering of stimulating light onto other low-signal portions of the plate during scanning, which is called flare, can make it impossible to detect latent-image signals below a certain level. In addition, various sources of noise (see Section 3.3.4) produce a "noise floor" below which no useful signal is detectable [62, 72].

The crucial point with $\mathrm{CR}$ is that one does not need to find a new detector to experiment with dose increase or reduction. The same plate and scanner will work under a large variety of exposure conditions (a range of $10^{4}$ ) without changing anything. This is not true for S/F systems, which are limited to about 30-40:1.

\subsubsection{Spatial Resolution}

The main factor limiting the spatial resolution in both $\mathrm{S} / \mathrm{F}$ and $\mathrm{CR}$ systems, is the scatter of optical photons within the phosphor layer. It is the scattering of stimulating laser beam in $\mathrm{CR}$ rather than emitted phosphor light in $\mathrm{S} / \mathrm{F}$, that determines system sharpness. Since the spread of laser light spot within IP increases with depth in the phosphor layer, the spatial resolution response of $\mathrm{CR}$ depends on both the initial laser beam diameter and the thickness of the turbid phosphor layer [72]. The trade-off between absorption and resolution is a compromise between detecting more $\mathrm{X}$-ray quanta and maintaining sharpness, the result of which is a family of plates adapted to different applications.

"After glow" is another important factor affecting the spatial resolution in CR systems. Storage phosphors continue emitting light for a short time after stimulation is stopped. For the materials commonly used in CR today, luminescence decay time is around $0.7-0.8 \mu \mathrm{sec}[56]$. As the laser beam moves across the plate, the light being collected from the current position can be mixed with the still "glowing" emission from previous laser positions. This causes a one-dimensional smear or resolution loss in the output signal. At low scan speeds, this is not an issue, but spatial resolution will be affected as the dwell time per pixel (in the range of $1-6 \mu$ sec today) starts to approach the luminescence decay time. Although there are image preprocessing techniques to unscramble this effect mathematically, they do so only with a loss in SNR (i.e., image quality) [72]. Thus, the long-standing drive toward faster scanners has been limited by a natural physical limit of existing phosphors. 


\subsubsection{Contrast Resolution}

As already discussed, the coupling of detector and display functions in film is a major limiting factor for conventional film radiography in which it is required to choose a film whose properties are a compromise between the requirements of photon detection and image display. Hence, separation of detector and display functions, as in CR, leads to optimization rather compromise since each function is optimized separately. Therefore, the contrast or grayscale resolution in CR is much greater than $\mathrm{S} / \mathrm{F}$ [62]. Since overall image quality is determined largely by a combination of spatial and contrast resolutions, the superior contrast resolution of CR can often compensate for its lesser inherent spatial resolution. By manipulating the image contrast and brightness (window-level $[\mathrm{W} / \mathrm{L}]$ ), small features on the image often become more apparent [72].

\subsubsection{Noise}

Noise is the random variation of some output signal around the mean value predicted by its I/O relationship. Both X-ray dose-dependent and fixed noise affect quality of CR images.

The predominant factor among fixed-noise sources in CR systems is IP structural noise which is due to the non-uniformity of phosphor particle distribution, with fine particles generating less noise [72]. Other factors include noise in the electronic chain, laser power fluctuations, and quantization noise in the ADC conversion process [62]. Note that electronic chain noise pertains practically to every CR scanner component as a potential noise contributor, some more serious and some less.

The dose-dependent noise components can be classified into X-ray quantum noise or mottle and light photon noise. The exposure-related noise is primarily the quantum noise inherent in the X-ray beam, being inversely proportional to the detector X-ray dose absorption. Light photon noise is a temporal fluctuation of photoelectrons arising in the process of photoelectric transformation of PSL light at the surface of PMT. The CR noise components are also discussed in the next chapter, Section 4.6.2.

CR systems tend to be limited by SNR considerations whereas S/F systems are contrast-limited [72]. And as discussed in Section 3.3.1, CR reader noise tolerance is the limiting factor in determining the lowest acceptable dose.

In contrast to the public beliefs, CR devices are not inherently lower-dose systems than $\mathrm{S} / \mathrm{F}$, except for a reduction in retakes due to over- and under-exposures with $\mathrm{S} / \mathrm{F}$. In fact, from a dose-efficiency standpoint, $\mathrm{CR}$ and $\mathrm{S} / \mathrm{F}$ systems are actually fairly comparable. Many studies [70] have shown that to achieve the same objectively measured image quality as $\mathrm{S} / \mathrm{F}$ systems, CR usually needs a bit more X-ray exposure, most likely due to the loss of about half of the potential signal in CR plate as prompt emission during the exposure, which was discussed previously. 


\subsection{Quality Improvement and Artifacts}

Since CR is more tolerant than film to a broad range of exposures, and image quality in CR tends to improve steadily with dose, unlike in S/F, CR exposures and image quality should be monitored by administrating quality assurance (QA) and quality control (QC) routines. Procedures to guide the diagnostic radiological physicist in the evaluation and continuous quality improvement of PSP imaging practice were published by AAPM Task Group 10, Report 93 in 2006 [64].

Artifacts in CR images can arise from a variety of sources including those related to the IPs, scanner, and image processing. Although they have been minimized by the latest technology improvements, older systems can still be prone to them [63].

High sensitivity of CR plates makes them extremely sensitive to scattered radiation and unintentional exposures. The natural background radiation can produce a noise field (a stored latent image) in the plates. So, it is always advisable to erase plates that have not been used for a while before starting new exposures. In addition, two-stage erasure is recommended after high or prolonged exposures. Inadvertent double exposures can occur with the current CR systems, potentially masking lowdensity regions in the image. Such artifacts are more difficult to detect than with S/F system due to $\mathrm{CR}$ wide dynamic range [72]. To avoid artifacts caused by physical damages such as cracks or wear-and-tear, IPs and cassettes should not be stacked atop one another. Laser scanning artifacts, seen as a linear artifact across the image, can still occur with the current CR readers, caused by dust on the light source. Frequent cleaning of the laser and light guide apparatus as well as IPs can prevent such artifacts.

\subsection{New CR Developments}

Since the inception of CR commercially in the early 1980's, it has improved significantly in overall design and performance characteristics, including reduction in the physical size of the scanning unit, increased reader plate handling capacity, and better image quality. The advancements have been achieved through a combination of changes in the IP, scanner, and image processing algorithms [63, 56].

The newer IPs have improved image quality by using smaller phosphor grain size which diminishes the fixed noise, while increasing the packing density of phosphor particles counteracts a decrease in PSL. On the other hand, a thinner protective layer on the plates reduces noise and improves spatial resolution as a result of diminished laser beam scattering. In addition, the quantity of phosphor coated onto the plate is increased for sensitivity and durability, resulting in the same resolution as with previous plates.

The idea of detecting emitted light from both sides of the plate has proved to extract more signal, and thus improved SNR [62]. In this development, the substrate 
of the plate is made transparent, and a second set of light collection optics, along with a photodetector and electronics, is added on the opposite side of the plate.

Needle-phosphor plates have demonstrated improved spatial resolution and DQE over powder phosphors. Rather than being coated onto a substrate like conventional phosphors and current powder-based storage phosphors, structured (needle) phosphors are grown under carefully controlled temperature, pressure, and mechanical conditions to form long crystalline rods or needles roughly perpendicular to the substrate [62]. The needles keep any luminescence generated inside them traveling along the needles, acting like fiber-optic light guides, which minimizes lateral scattering of light photons and helps to maintain contrast sensitivity.

Modern flying-spot scanners still consist of discrete components mostly. In addition, flying-spot scanners are subject to the "speed limit" of IP's luminescence decay time, which sets a minimum pixel dwell time to avoid blurring. In line-scanning readers, the scanning head moves over a stationary plate, in which the laser light stimulates one line at a time and PSL is acquired by a charge-coupled-device (CCD) linear array photodetector [72]. In this design, many of the discrete components found in flying-spot scanners are integrated into the scanning head. Depending on the design, even the analog electronics and ADC converter can be incorporated into the head, resulting in significant increase in scanning speed [62]. 


\section{Chapter 4}

\section{Image Quality Assessment of a Computed Radiography System}

\subsection{Introduction}

Since the introduction of computed radiography systems into general commercial use, improvements in image plates and readers have brought about incremental gains in imaging performance. CR 7400 is the latest generation of Kodak desktop-sized photostimulable phosphor image readers which was used in this project. The imaging performance characteristics of the system were studied by measuring the presampled Modulation Transfer Function (MTF), Noise Power Spectrum (NPS), Relative Standard Deviation of Noise (RSD), Noise Equivalent Quanta (NEQ), and Detective Quantum Efficiency (DQE).

\subsection{Imaging System}

CR 7400 is popular in dental radiology and is based on the well-known CR technology consisting of granular phosphor plate and single-side flying spot reader. Table 4.1 summarizes the specifications of the unit and imaging plate used in the study.

\subsection{Exposure Geometry}

The objective of this project was to detect iodinated contrast agent in a small animal tumor model and ultimately have a similar approach in visualizing the iodinated contrast agent in a mouse tumor. Thus, various parameters of the exposure geometry were to be optimized to meet the requirements of imaging a mouse. Imaging the angiogenic circulation/zone in mice is extremely challenging. Since the image noise is proportional to $\triangle x^{-2}(\triangle x$ : image pixel size), the X-ray flux needs to be high enough to improve signal-to-noise ratio. Moreover, the physiological motion in mice is at least ten times faster than humans. The delivered dose should also be kept minimum to allow longitudinal studies. In conclusion, special strategies must be considered, including short exposures to limit the motion blur and high X-ray flux to 
Table 4.1: CR 7400 specifications.

\begin{tabular}{cc}
\hline Parameter & Description $/$ Value \\
\hline Manufacturer & Kodak \\
Model & CR 7400 \\
IP Type & HR \\
Phosphor Type & Granular; $\mathrm{BaFBr}_{0.85} \mathrm{I}_{0.15}: \mathrm{Eu}^{2+}$ \\
Phosphor Thickness $(\mu \mathrm{m})$ & 300 \\
Phosphor Effective Density $\left(\mathrm{gr} / \mathrm{cm}^{3}\right)$ & 2.80 \\
Reader Type & Single-side, Flying-spot \\
Image Area $\left(\mathrm{cm}^{2}\right)$ & $15 \times 30$ \\
Image Array Size & $2996 \times 5776(\mathrm{HR}), 1500 \times 2888(\mathrm{HS})$ \\
Pixel Pitch $(\mu m)$ & $50(\mathrm{HR}), 100(\mathrm{HS})$ \\
Image Depth $(\mathrm{bits})$ & 12 \\
\hline
\end{tabular}

Notes: HR: High Resolution, HS: High Speed.

improve signal-to-noise ratio. Micro-focus X-ray tubes are the most common tubes used in small animal studies. The photon flux from a given focal spot is proportional to the focal spot size $\left(\sim f_{s}^{n}\right)$, where $n$ ranges between 1 and 2 . It is also linearly related to the current and the maximum continuous current is limited by the thermal capacity of the focal spot. Thus, micro-focus X-ray sources require longer exposure times to achieve comparable flux than the large-focal-spot X-ray tubes. An increase in the number of photons is required to provide a higher signal-to-noise ratio, leading to improved low-contrast resolution. On the other hand, a large-focal-spot X-ray tube produces higher flux, but the penumbral blur from a large focal spot reduces the spatial resolution (Figure 4.1). One can reduce this blur by moving the tube further from the object and detector, but this, in turn, reduces the flux at the detector.

Assuming fixed $\mathrm{kVp}$ and $\mathrm{mA}$, the flux $(\phi)$ can be optimized by careful trade-off between the source-to-detector distance $(S D D)$ and resolution (res). The resolution limit due to penumbral blurring $(b)$ in the projection plane is related to the focal spot diameter $\left(f_{s}\right)$, the object-to-detector distance $(O D D)$ and the source-to-detector distance by

$$
b=\frac{O D D}{S D D-O D D} f_{s} .
$$

To first order, the current, and therefore the flux of an X-ray tube is proportional to the focal spot size. At a given $S D D$,

$$
\phi \propto \frac{f_{s}^{n}}{S D D}, \quad 1<n<2
$$

where $n$ will be determined by fitting available tube focal spots at desired $S D D$ to obtain the maximum flux. The flux will be optimized when $S D D$ is set such that the blur equals the desired resolution $(b=r e s)$. Solving Equation 4.1 for this condition 


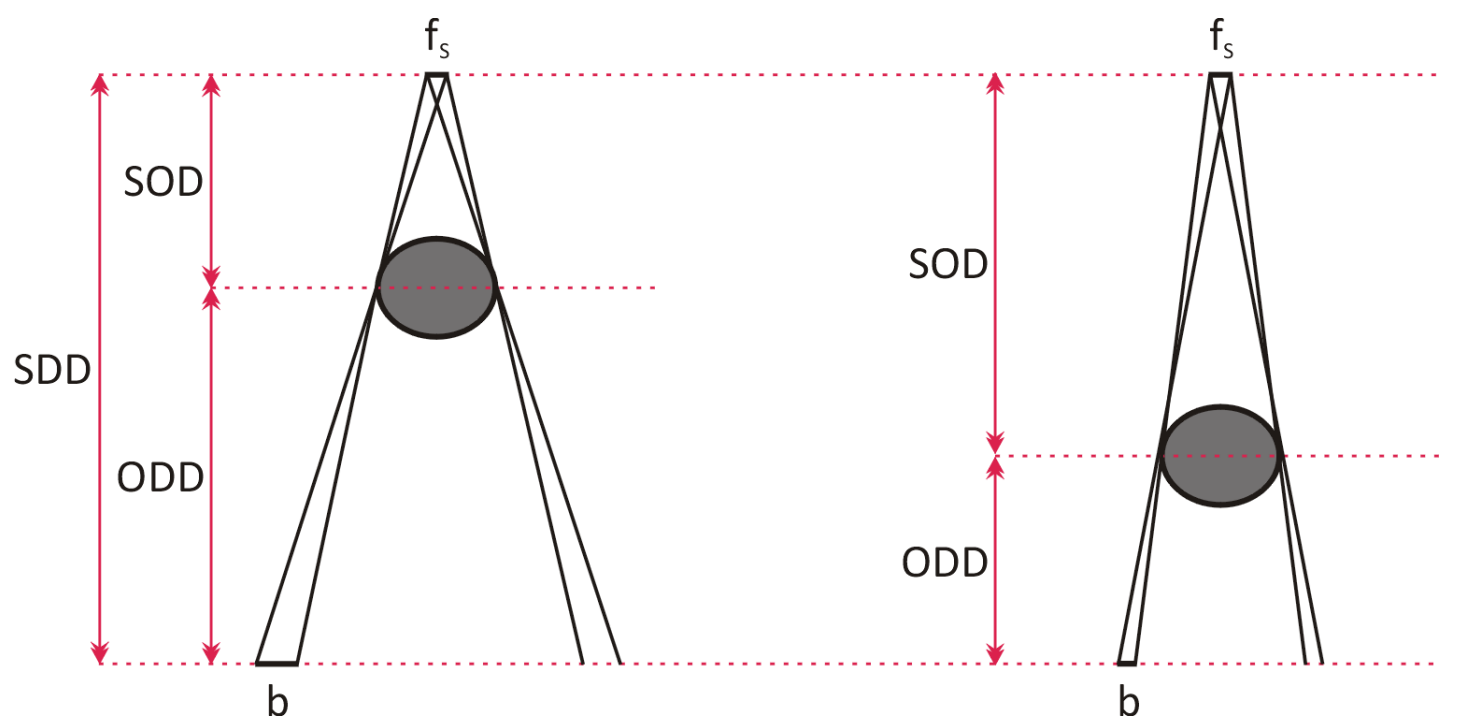

Figure 4.1: The effect of penumbral blurring with respect to source-toobject and object-to-detector distances.

and substituting $S D D$ in Equation 4.2 yields the expression of maximum flux for a given focal spot at a desired resolution $[8,9]$ :

$$
\phi \propto \frac{f_{s}^{n}}{\left(\left(\frac{f_{s}}{r e s}+1\right) O D D\right)^{2}} .
$$

\subsection{Exposure Conditions and Physical Characterization}

The images for evaluation of the unit were acquired using a high-frequency Varian G-1092 rotating anode X-ray tube $(0.6$ and $1.2 \mathrm{~mm}$, nominal focal spots; $0.7 \mathrm{~mm}$ $\mathrm{Al}$ inherent filtration [67]) in a B-160H housing (Varian Medical Systems, Salt Lake City, UT). The tube employed a Duocon M-100 X-ray collimator (2 mm Al, inherent filtration; The Machlett Laboratories Inc., Stamford, CT) attached to the tube front window. The accuracy of voltages applied were verified to be within $5 \mathrm{kV}$. All images were acquired with the small focal spot and no added filtration. After acquisition, the images were transferred to a research computer as 12-bit, raw/unprocessed data for analysis.

Prior to processing any images, flat-field correction was performed on all the acquired images according to [58]

$$
P_{c}^{X}(i, j)=\left(P_{u c}^{X}(i, j)-O_{m}(i, j)\right) \frac{M}{G_{n}^{X}(i, j)-O_{m}(i, j)},
$$

where $P_{c}(i, j)$ and $P_{u c}(i, j)$ are the corrected and corresponding uncorrected pixel values at coordinate $(i, j)$ acquired at exposure $X$, respectively, $G_{n}(i, j)$ is the gain 
image acquired as the average of $n$ sequential uniform flat-filed images at exposure $X$, $O_{m}(i, j)$ is the offset image acquired as the average of $m$ sequential flat-filed unexposed (dark-field) images, and $M$ is the mean pixel value of the offset-corrected gain image $\left(\left\langle G_{n}^{X}(i, j)-O_{m}(i, j)\right\rangle\right)$. Here, $n$ and $m$ are chosen 5 for all studied exposures.

The response function of the system was determined by exposing the imaging plate to a wide range of uniform X-ray exposures acquired at $80 \mathrm{kVp}$ and $1,5,10$, and $15 \mathrm{mAs}(5.22,22.93,45.68$, and $68.27 \mathrm{mR})$. First, exposures, each repeated five times, were measured using a calibrated ionization chamber (Model 2026C, 20X6-60E, ionization chamber, Radcal, Monrovia, CA) positioned at the location of the plate (plate being removed) and approximate center of the beam axis. Then, the plate was placed back, exposed at the same range of exposures, and at each exposure which was repeated five times, the mean pixel value of central $128 \times 128$ ROI in the image was estimated. System response function was then computed from a linear fit of the averaged mean pixel values versus measured average exposures at each mAs setting. The projection geometry is shown in Figure 4.2. The linear fit to the data uses a zero intercept, proving that pixel values behind $\mathrm{Pb}$-masked areas were essentially zero.

\subsection{Presampled Modulation Transfer Function (MTF)}

The schematic of data acquisition set-up similar to the one recommended by the IEC [1] and discussed in Section 4.3 is shown in Figure 4.2.

An edge test device (TX5, IBA Dosimetry, Scanditronix Wellhöffer, Schwarzenbruck, Germany) made of a $1-\mathrm{mm}( \pm 5 \mu \mathrm{m})$ thick, $8 \times 10 \mathrm{~cm}^{2}$ tungsten slab, polished on one side and fixed on a 3-mm thick lead frame on the other three sides, was placed in contact with the plate and aligned with the central axis of X-ray beam. The edge was tilted by $2^{\circ}-3^{\circ}$ with respect to the direction along which the MTF was being measured. Since the preliminary experiments demonstrated insensitivity of measured MTF to the exposure level (excluding very underexposed edge images), the edge test device was imaged once at $80 \mathrm{kVp}, 15 \mathrm{mAs}(\sim 68.27 \mathrm{mR})$, from which ten independent ROIs $(256 \times 256$ for $100 \mu \mathrm{m}$ resolution, $512 \times 512$ for $50 \mu \mathrm{m}$ resolution $)$ encompassing the edge were extracted. The size of ROIs was chosen such that ROIs were small enough to ensure the uniformity of exposure and large enough to contain sufficient low-frequency data. The MTFs were measured in both horizontal (laser-scan) and vertical (plate-scan) directions adopting the well-known edge technique similar to Samei and Flynn [60]. In summary, the angle of the edge was first determined with 0.02 degree accuracy using Hough transformation. The $2 \mathrm{D}$ data in the region of the edge were then projected along the determined angle with respect to their distances to the edge into a one-dimensional estimate of the edge spread function (ESF) using bins of 0.1 pixels. The ESF data were smoothed using a moving Gaussian-weighted polynomial fit, and differentiated to obtain the line spread function (LSF). Finally, the LSF was windowed with a spectral estimation function and Fourier transformed to obtain the presampled MTF $[60,59,58]$. The MTF was then normalized to its - 


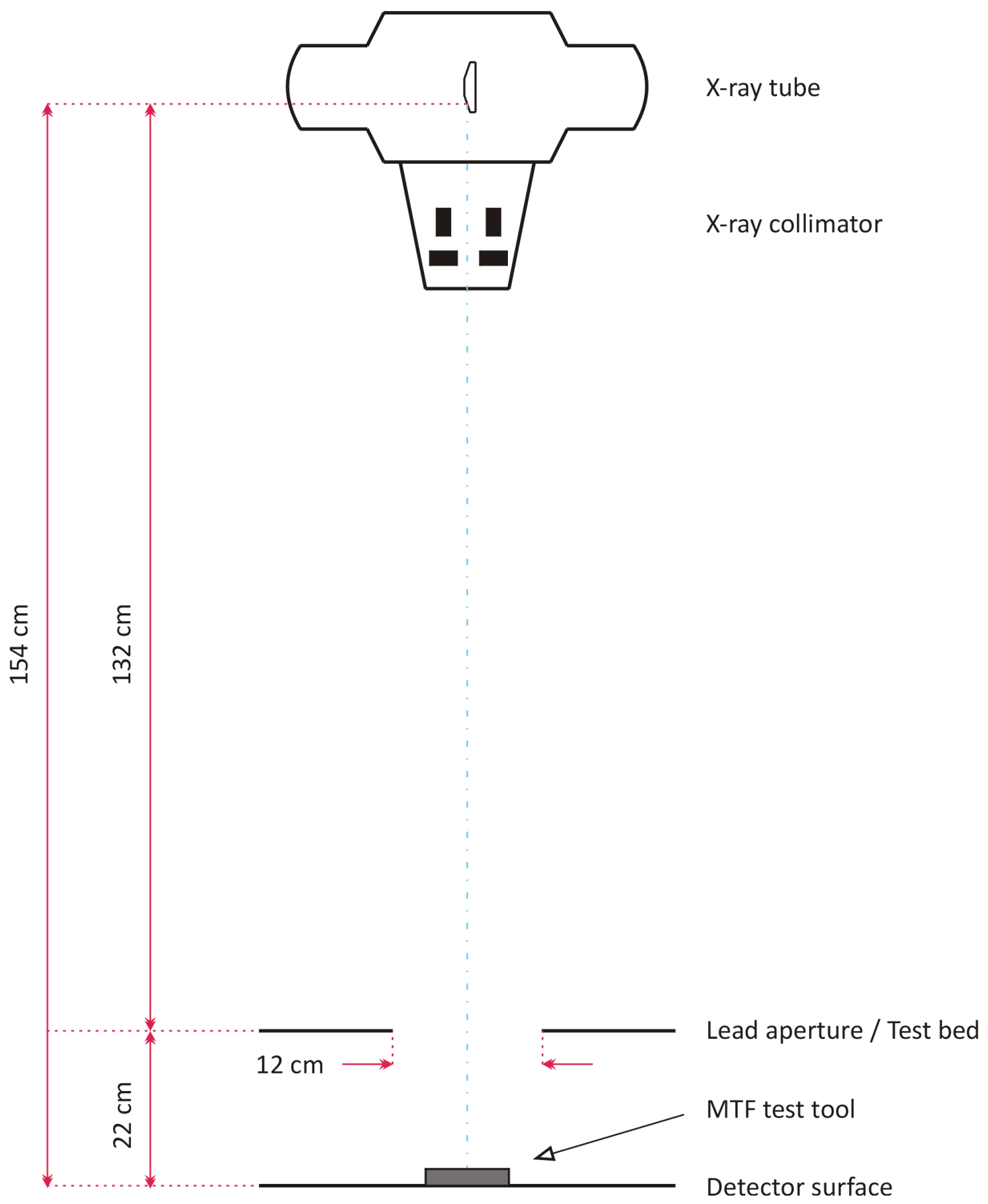

Figure 4.2: Schematic of the data acquisition set-up for system response function and MTF measurements. 
value at zero frequency and the negative frequency components of Fourier transform were discarded. In the end, the measured MTFs were averaged to represent one final MTF for each direction.

\subsection{Noise Characteristics}

\subsubsection{Noise Power Spectrum (NPS)}

The NPS measurements were made by Fourier analysis of uniformly-exposed radiographs. Uniform flat field images were acquired at $80 \mathrm{kVp}$ and $1,5,10$, and 15 mAs $(5.22,22.93,45.68$, and $68.27 \mathrm{mR})$. The central portion of each image, excluding the edges of the image, was subdivided into multiple $256 \times 256$ regions. The noise spectrum within each region was computed using a 2D fast Fourier transformation, as shown in [33]

$$
N P S(u, v)=\frac{|F T[I(x, y)]|^{2}}{\overline{\text { signal }}^{2}} N_{x} N_{y} \Delta_{x} \Delta_{y},
$$

where $u$ and $v$ denote spatial frequency in the $x$ and $y$ directions, $I(x, y)$ is the signal at detector pixel $(x, y)$, FT represents the 2D Fourier transform, $N_{x}$ and $N_{y}$ are the number of pixels in the $x$ and $y$ directions, $\Delta_{x}$ and $\Delta_{y}$ are the pixel sizes in the $x$ and $y$ directions and $\overline{\text { signal }}$ represents the average of $I(u, v)$ over the region used to evaluate the NPS. The spectra from all regions were averaged to obtain the overall $2 \mathrm{D}$ NPS. The NPS in the horizontal and vertical directions were extracted by averaging the central \pm 10 horizontal/vertical lines, excluding the values along the axes. As an alternative to calculate the fraction in Equation 4.5, the data within each ROI could be first converted to relative noise units by dividing them by their mean value $[59,58]$, then Fourier transformed, and their magnitudes squared. Finally, in order to reduce the uncertainty of the 2D NPS, the results of NPS measurements at each exposure, which was repeated five times, were averaged.

\subsubsection{Relative Standard Deviation (RSD)}

Noise was also studied by a RSD analysis. The same central ROIs used in NPS calculations were also used to perform RSD analysis. RSD (i.e., standard deviation divided by average signal) was calculated for each central ROI at each exposure. The RSDs were averaged to obtain final RSD, then squared and linear-fitted according to $[13,55,50]$

$$
R S D^{2}=\left(\frac{\sigma_{t o t}}{x}\right)^{2}=\frac{\alpha}{x}+\beta+\frac{\gamma}{x^{2}}
$$

where $x$ is the $\mathrm{X}$-ray exposure, and $\alpha, \beta$, and $\gamma$ represent contributions from the quantum (Poisson) noise source, dose-dependent noise source (multiplicative), and dose-independent noise source (additive), respectively. 
Equation 4.6 is derived from the definition of total variance $\sigma_{t o t}^{2}$, defined by Equation 4.7. In fact, $\sigma_{t o t}^{2}$ can be described as the sum of three terms related to Poisson, multiplicative, and additive noise. The first two terms are proportional to exposure (Poisson noise) and square of exposure (multiplicative noise), respectively, and the last one is independent of exposure.

$$
\sigma_{\text {tot }}^{2}=\sigma_{\text {poi }}^{2}+\sigma_{m u l}^{2}+\sigma_{\text {add }}^{2}=\alpha x+\beta x^{2}+\gamma \text {. }
$$

In a $\mathrm{CR}$, the additive factor can be interpreted as the electronic noise (e.g., dark current), whereas the multiplicative component is related to the structure noise due to variations in sensitivity across the imaging plate.

\subsubsection{Noise Equivalent Quanta (NEQ)}

NEQ expresses the apparent number of quanta per unit area contributing to an image if all the noise sources in the system are assigned to "photon-counting" statistics [14]. In a real imaging system, the number of detected X-ray quanta is always lower than quanta incident upon the detector due to the fact that detection of the incident quanta is incomplete and there is also degradation of the input information due to noise processes in the image formation, recording stages and contrast loss processes [24]. The NEQ, as shown in Equation 4.8, yields the image's output signal-to-noise ratio, expressed in terms of quanta, as a function of spatial frequency. This measure is essentially the number of input quanta per unit area that an ideal detector would have needed to yield the same signal-to-noise ratio, as the actual exposure produces in the real detector [14].

$$
N E Q(f)=\frac{M T F^{2}(f)}{N P S(f)} .
$$

\subsection{Detective Quantum Efficiency (DQE)}

DQE is a measure of the SNR transfer efficiency of an X-ray imaging system and as such provides a measure of exposure efficiency. It was computed using [59]

$$
D Q E(f)=\frac{S N R_{\text {out }}^{2}}{S N R_{\text {in }}^{2}}=\frac{G \times N E Q(f)}{q \times X},
$$

where NEQ(f) is the measured noise equivalent quanta at a given exposure $X, G$ is the gain factor, set equal to unity since the image data were linearized prior to any processing and the NPS was computed from the relative noise variations. $q$ is the square of the ideal signal-to-noise ratio per exposure expressed in $\frac{\text { photons }}{m m^{2} m R}$ and has a different definition when considering an ideal "energy-integrating" or "photoncounting" detector. 
Assuming an ideal "energy-integrating" detector, the $q$ values were calculated from the first and second moments of the energy spectrum, accounting for both characteristic and bremsstrahlung radiations, using Equation 4.10 [59]

$$
q=\frac{\left(\int_{0}^{k V p} \phi(E) E d E\right)^{2}}{X_{\phi} \int_{0}^{k V p} \phi(E) E d E}
$$

which is based on a semi-empirical model for the differential energy spectrum produced by an X-ray tube. In this model, X-ray source spectra were simulated using the spectrum processor of IPEM Report-78 based on the work by Birch and Marshall [40]. $E$ is the photon energy, $\phi(E)$ is the X-ray spectrum (photons $/ \mathrm{mm}^{2} \mathrm{keV}$, and $X_{\phi}$ is the exposure in $m R$ associated with the $\phi(E)$. Values for $X_{\phi}$ were obtained using [32]

$$
X_{\phi}=\frac{C_{R}}{W} \int_{0}^{k V p} \phi(E) E\left(\frac{\mu_{e n}}{\rho}\right)_{\text {air }} d E .
$$

$X_{\phi}$ values were obtained by computing the energy absorbed in air for the spectrum $\phi(E)$ using mass energy-absorption coefficient data from the National Institute of Standards and Technology (NIST) [39]. The energy absorbed in air was then converted to charge using a $W$ value of $33.97 \mathrm{~J} / \mathrm{C}$ (i.e., ev/ion pair). $C_{R}$ is a unit conversion factor $\left(=6.201 \times 10^{-10} \mathrm{RgJ} / \mathrm{keV} \mathrm{C}\right)$.

If an ideal "photon-counting" detector is assumed, the $q$ values can be calculated as $[59]$

$$
q=\frac{1}{X_{\phi}} \int_{0}^{k V p} \phi(E) d E .
$$

Based on the tentative recommendations of the IEC working group on DQE [37], "energy-weighted" $q$ values were used in the DQE calculation. However, as reported by Samei and Flynn [59], the difference between calculated $q$ values in Equations 4.10 and 4.12 was $5 \%$ at the most. 


\section{Chapter 5}

\section{Dual-energy Subtraction Technique}

\subsection{Introduction}

The mass attenuation coefficient of a material $\frac{\mu(E)}{\rho}$ may be decomposed through an appropriate linear set of basis functions $\left\{f_{i}(E), i=1,2, \ldots, n\right\}$. This provides a small number of empirical parameters $\left\{a_{i}(E), i=1,2, \ldots, n\right\}$, such that $\mu(E)$ is expressed by the following linear combination of associated basis functions $[18,5]$ :

$$
\frac{\mu(E)}{\rho} \approx a_{1} f_{1}(E)+a_{2} f_{2}(E)+\ldots+a_{n} f_{n}(E) .
$$

As already expressed in Chapter 2 (Section 2.3), in the X-ray energy range employed for diagnostic radiography, the total mass attenuation coefficient of a material through which X-ray photons propagate, is a combination of contributions mainly from the photoelectric effect and Compton scattering plus a smaller amount from Rayleigh scattering, all of which are energy dependent and make logical choices for basis functions. Figure 2.6 demonstrates the relative contributions to and the energy dependence of the mass attenuation coefficients for soft tissue, iodine and barium.

The basis functions used for decomposition comprise a vector space and naturally the question arises what constraints limit the dimensionality of the vector space. Up to the level of calculation precision, the attenuation coefficient accuracy is theoretically improved as the dimensionality of the basis function vector is increased. However, Lehmann and Alvarez [42] have demonstrated that monochromatically, the major reduction in the calculated attenuation coefficient error comes from using a twodimensional vector space. Use of higher dimensionality results in only infinitesimally increased accuracy above the two-dimensional fractional RMS error of $0.4 \%$ found for atomic numbers from 1 to 25 and energies from 40 through $100 \mathrm{keV}$. This error is roughly comparable to the level of quantum noise in digital radiography, setting an upper bound on the physically realizable dimensionality at two. These observations agree with the fact that decomposition via photoelectric and Compton scattering basis functions have been found sufficient to yield mass attenuation coefficients for body materials within $1 \%[54,69]$. Consequently, Equation 5.1 is simplified to [43]

$$
\frac{\mu(E)}{\rho} \approx a_{p e} f_{p e}(E)+a_{C} f_{C}(E)
$$

where $a_{p e}$ and $a_{C}$ are characteristic constants of the material and $f_{p e}$ and $f_{C}$ are the energy dependencies of photoelectric absorption and Compton scattering, respectively. 
$a_{C}$ is the electron mass density $\left(N_{g}\right)$, described as

$$
a_{C}=N_{g}=\frac{Z}{A} \times 6.022 \times 10^{23},
$$

where $Z$ and $A$ are the atomic number and mass, respectively. $f_{C}$ is the Klein-Nishina function,

$$
f_{C}(\gamma)=C_{0}\left\{\frac{1+\gamma}{\gamma^{2}}\left[\frac{2(1+\gamma)}{1+2 \gamma}-\frac{1}{\gamma} \ln (1+2 \gamma)\right]+\frac{1}{2 \gamma} \ln (1+2 \gamma)-\frac{1+3 \gamma}{(1+2 \gamma)^{2}}\right\}
$$

where $\gamma=\frac{E}{510.975 \mathrm{keV}}$ and $C_{0}=2 \pi\left(\frac{\mu_{0} e^{2}}{4 \pi m}\right)^{2}=2 \pi r_{0}^{2}$. Photoelectric absorption can be approximated as

$$
a_{p e} f_{p e} \approx N_{g} C_{p} \frac{Z^{3.8}}{E^{3.2}},
$$

where $C_{p}=9.8 \times 10^{-24}$ and $E$ is measured in $k e V$.

By measuring X-ray attenuation at various energies, separation of the total attenuation into its basic components, photoelectric and Compton scattering, is permitted through various numerical methods. The resulting parameters $\left(a_{i}\right)$ can then be used to determine elementary tissue properties. The method of obtaining these two elementary tissue properties using images acquired with two distinct X-ray spectra is termed dual-energy decomposition.

\subsection{Theory}

Since the objective of the current project is to extract soft tissue (water) and contrast agent (iodine) information from the radiographic images, an alternate and more convenient representation of total attenuation is to use the energy dependence of the attenuation of water (plexiglas) and contrast agent (iodine) for basis functions.

There are two general methods to extract soft tissue and contrast agent information from radiographic images at different energies: subtraction and decomposition.

\subsubsection{Dual-energy Subtraction}

Linear subtraction theory assumes that the high- and low-energy input spectra are monochromatic and basically only two distinct materials exist within the object. The relation between incident and exiting radiation intensities is:

$$
\begin{aligned}
& \ln \left(\frac{I^{H}}{I_{0}^{H}}\right)=-\mu_{w}^{H} t_{w}-\mu_{i o}^{H} t_{i o} \\
& \ln \left(\frac{I^{L}}{I_{0}^{L}}\right)=-\mu_{w}^{L} t_{w}-\mu_{i o}^{L} t_{i o}
\end{aligned}
$$


where $t_{w}$ and $t_{i}$ are water and iodine thicknesses, respectively, with their corresponding linear attenuation coefficients $\mu_{w}$ and $\mu_{i o}$, acquired at high and low energies $(H$ and $L$ ). Simple subtraction of the two images yields:

$$
\ln \left(\frac{I^{H}}{I_{0}^{H}}\right)-\ln \left(\frac{I^{L}}{I_{0}^{L}}\right)=\left(\mu_{w}^{L}-\mu_{w}^{H}\right) t_{w}+\left(\mu_{i o}^{L}-\mu_{i o}^{H}\right) t_{i o} .
$$

To enhance the contrast between $t_{w}$ and $t_{i o}$, the high- and low-energy input spectra must be chosen such that $\frac{\mu_{w}^{L}-\mu_{w}^{H}}{\mu_{i o}^{L}-\mu_{i o}^{H}}>\frac{\mu_{w}}{\mu_{i o}}$, where $\mu_{w}$ and $\mu_{i o}$ are the two respective attenuation coefficients at a certain intermediate energy $\left(E_{L}<E<E_{H}\right)$. Equation 5.6 may be easily solved through linear algebra to yield the thicknesses of water and iodine. However, the resulting solution holds only as well as the validity of the assumption made regarding the monochromatic spectra. For a typical radiographic spectral source, this assumption is, of course, not realistic.

Simple or weighted subtraction of high- and low-energy images for polychromatic spectra and diagnostic objects primarily yields qualitative visual information of where the high atomic number materials (iodine here) reside, but does not provide quantitative information on tissue (water here) properties. Decomposition, on the other hand, provides system independent quantitative tissue properties through comparison of observed tissue attenuation factors with those of standardized reference materials used in a calibration procedure.

\subsubsection{Dual-energy Decomposition}

Dual-energy decomposition implementing Alvarez and Macovski's method [3], decomposes the attenuation coefficient into a set of linear basis functions, the requisite coefficients being determined through a self-calibration process. In projection radiography, the transmitted intensity measured by the detector at energy $E$ is essentially a line integral of the attenuation coefficient over the X-ray path length $(s)$ : $\int \mu(x, y, z ; E) d s$, assuming narrow beam geometry (negligible scattering contribution).

As already shown in Equation 5.2, since the attenuation coefficient $\mu$ can be decomposed into a combination of photoelectric absorption and Compton scatter functions, this line integral can be written as

$$
\begin{gathered}
\int \mu(x, y, z ; E) d s=\int a_{p e}(x, y, z) f_{p e}(E) d s+\int a_{C}(x, y, z) f_{K N}(E) d s \\
=A_{p e} f_{p e}(E)+A_{C} f_{K N}(E) .
\end{gathered}
$$

The measured transmission for an input polychromatic spectral distribution $\phi(E)$ becomes:

$$
\frac{I}{I_{0}}=\int \phi(E) e^{-A_{p e} f_{p e}(E)-A_{C} f_{K N}(E)} d E
$$


where $I$ and $I_{0}$ are the transmitted and reference intensities, respectively.

The decomposition problem then reduces to recovering the photoelectric and Compton scatter basis function coefficients $a_{p e}(x, y, z)$ and $a_{C}(x, y, z)$ for each position along the path length. This is possible in CT where the tomographic procedure, utilizing many projections, removes material superimposition. However, in projection radiography, the integrated energy dependence along the projection path is not solely associated with one material. If only two unique materials were present, measurement of transmission values along the path would yield their respective thicknesses just as with energy subtraction. This is not true in projection radiography for more than two materials.

Therefore, for polychromatic spectra and more than two unique materials along the path length, the attenuation characteristics of the intervening material must be expressed relative to the set of basis functions chosen for the decomposition. These basis functions comprise a vector space in which attenuation characteristics of any material is a projection. The photoelectric and Compton scatter basis functions, a logical choice based on the physics of the radiographic process, are, nevertheless, not unique. Any non-singular linear combination of these two basis functions themselves span the two-dimensional vector space, so that the mass attenuation coefficient of material $\psi$ may be decomposed into that of two other materials $\alpha$ and $\beta$ : $\mu_{\psi}(E)=a_{\alpha} \mu_{\alpha}(E)+a_{\beta} \mu_{\beta}(E)$. Thus, any convenient choice of materials $\alpha$ and $\beta$ providing two non-singular basis functions may be chosen.

Equation 5.9 may be re-written in terms of the new basis function set for highand low-energy radiographic data as

$$
\begin{aligned}
& \frac{I^{H}}{I_{0}^{H}}=\int \phi_{H}(E) e^{-\mu_{\alpha}(E) t_{\alpha}-\mu_{\beta}(E) t_{\beta}} d E \\
& \frac{I^{L}}{I_{0}^{L}}=\int \phi_{L}(E) e^{-\mu_{\alpha}(E) t_{\alpha}-\mu_{\beta}(E) t_{\beta}} d E
\end{aligned}
$$

where

$$
\begin{aligned}
& \mu_{\alpha}(E)=a_{p e}(\alpha) f_{p e}(E)+a_{C}(\alpha) f_{K N}(E) \\
& \mu_{\beta}(E)=a_{p e}(\beta) f_{p e}(E)+a_{C}(\beta) f_{K N}(E),
\end{aligned}
$$

which are merely linear projections defining the new set of basis vectors. Since there exist high- and low-energy transmitted intensities for each projection path, it is possible to obtain an analytic solution for the material equivalent thicknesses $t_{\alpha}(x, y, z)$ and $t_{\beta}(x, y, z)$ if $\phi_{H}(E)$ and $\phi_{L}(E)$ are known. However, these spectral distribution functions are not known a priori, being contingent upon the X-ray tube operating conditions, inherent filtration and detector response characteristics.

The analysis in the chapter preamble, limiting the dimensionality of the basis function vector space for decomposition to two, did not account for polychromatic 
beam-hardening characteristics. Such effects can account for an RMS error of approximately $6 \%$ [35] in the determination of material equivalent thicknesses. Thus, it is necessary to increase the number of basis functions through higher order approximation to account for the additional complexity found in the observed data. It then becomes more pragmatic to solve the problem numerically by approximating the right hand side of Equation 5.10 with, for example, a set of polynomials in $t_{\alpha}$ and $t_{\beta}$. This constitutes the discussion in Section 5.5.

\subsection{Decomposition Calibration}

According to Equation 5.10, the basis functions may be conveniently chosen as long as they have non-identical attenuation functions of energy. Thus, the photoelectric and Compton scattering basis functions may be substituted for a more pragmatic set of basis materials in the calibration procedure. The calibration phantom has two separate parts, designed to mimic the soft tissue and iodine variations in a small animal such as mouse or rat. The first part (soft tissue phantom) is a step wedge made of a 3-cm-thick plexiglas block, divided into ten 3-mm-thick steps (Figure 5.1). The second part (iodine phantom) is also a step wedge, but with inverted steps, made of a 1.48-cm-thick plexiglas block, containing ten 1-mm-thick steps (Figure 5.2). It is filled with Omnipaque-240 solution $\left(\mathrm{C}_{19} \mathrm{H}_{26} \mathrm{~N}_{3} \mathrm{O}_{9} I_{3} ; \rho=1.28 \mathrm{gr} / \mathrm{cm}^{3}\right.$, GE Healthcare Inc., Princeton, NJ), an iodinated contrast agent, whose iodine concentration has been reduced from $240 \mathrm{mgr} / \mathrm{cm}^{3}$ to $10 \mathrm{mgr} / \mathrm{cm}^{3}$. The steps are chosen such that it provides a wide range of iodine projection thicknesses $\left(1 \sim 10 \mathrm{mgr} / \mathrm{cm}^{2}\right)$ including the minimum detectable projection thickness of iodine in breast tumors $\left(2 \sim 3 \mathrm{mgr} / \mathrm{cm}^{2}\right)$, reported so far $[23,22]$. The step wedges are mounted at right angle to each other in order to give a total of 121 calibration measurements including zero iodine projection thickness. The number of steps in the calibration phantom is dictated by the desired calibration procedure effort, material resolution, and limiting factors imposed by the geometry of projection such as the size of field of view. The greater the amount of calibration data, the better the resulting calibration coefficients.

The radiographic technique used in formation of calibration phantom images is exactly the same as that used to image the test mouse phantom (Section 5.6), so that the material equivalent image produced by the decomposition algorithm corresponds with the thickness of material in the calibration (reference) phantom. The engineering drawings of calibration phantoms are shown in Appendix A. 


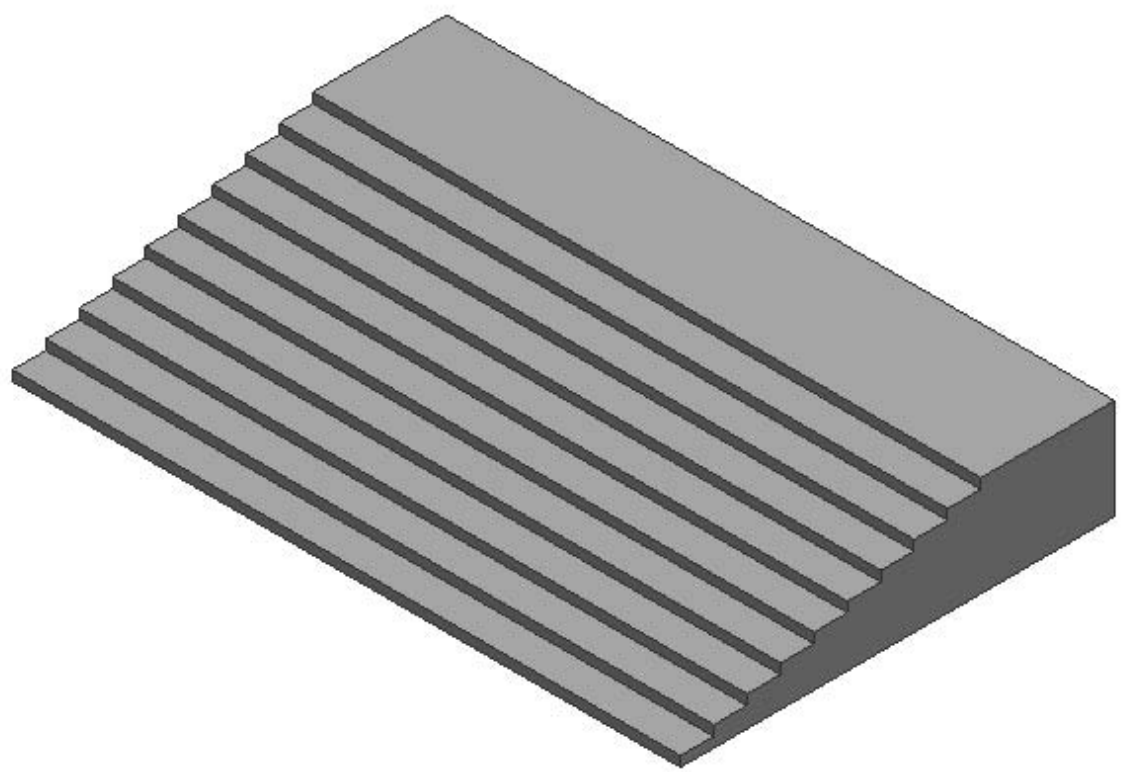

Figure 5.1: Step wedge 1 (soft tissue phantom).

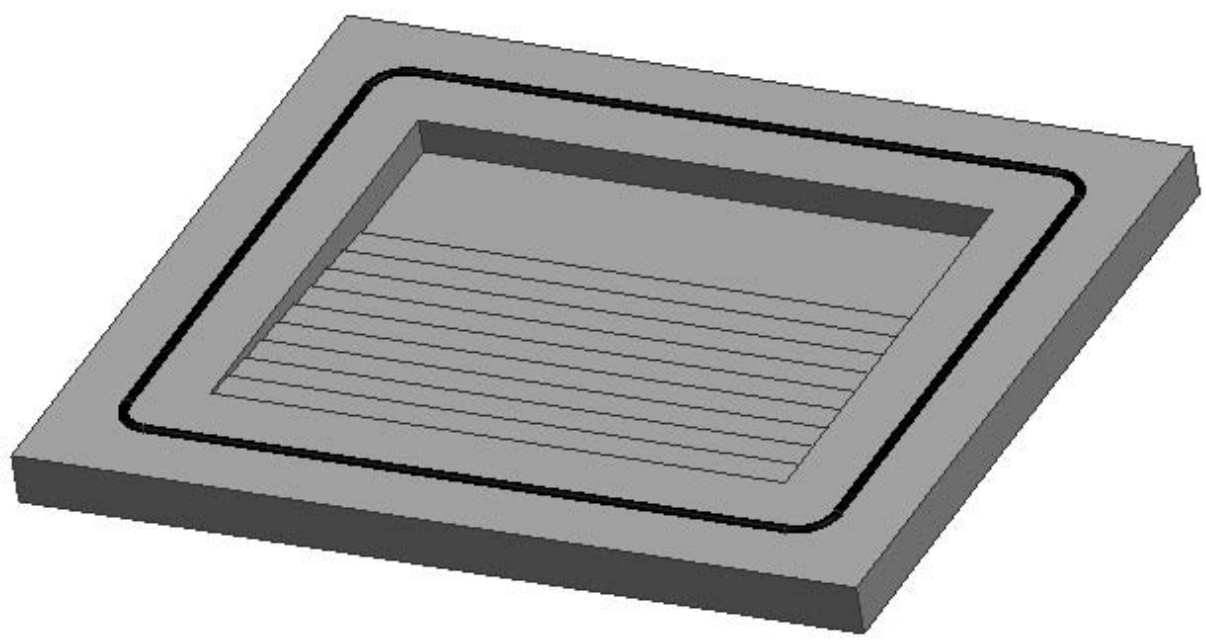

Figure 5.2: Step wedge 2 (iodine phantom). 


\subsection{Decomposition Algorithms}

The general form of the decomposition equations may be written as

$$
y_{i}\left(x_{i}\right)=\sum_{k=1}^{M} a_{k} X_{k}\left(x_{i}\right), i=1,2, \ldots, N
$$

where $X_{1}\left(x_{i}\right), X_{2}\left(x_{i}\right), \ldots, X_{M}\left(x_{i}\right)$ are arbitrary fixed functions of $x_{i}$, called basis functions. For dual-energy analysis, $y_{i}\left(x_{i}\right)$ values are simply either the high- or low-energy measured transmission for a certain combination of calibration material steps; plexiglas and iodine. Although the discussion of "Global Coefficient Algorithms" is deferred until next section, a polynomial set of the contrast agent (iodine) and plexiglas thicknesses, $t_{i o}$ and $t_{p l}$, will recast Equation 5.12 as

$$
\begin{gathered}
T_{E}\left(t_{i o}, t_{p l}\right)=\sum_{k=1}^{M} a_{k} X_{k}\left(t_{i o}, t_{p l}\right) \\
=a_{0}+a_{1} t_{i o}+a_{2} t_{p l}+a_{3} t_{i o} t_{p l}+a_{4} t_{i o}^{2}+a_{5} t_{p l}^{2}+\ldots,
\end{gathered}
$$

where the measured transmissions for the high- and low-energy images, $T_{H}$ and $T_{L}$, are used with the corresponding thicknesses of contrast agent and plexiglas in the calibration phantom to determine the appropriate basis function coefficients $\left(a_{k}\right)$ for equations $i=1,2, \ldots, N$. For this set of linear equations defined through the calibration procedure, one attempts a least-squares fitting of the calibration data by defining a merit function:

$$
\chi^{2}=\sum_{i=1}^{N}\left[\frac{y_{i}-\sum_{k=1}^{M} a_{k} X_{k}\left(x_{i}\right)}{\sigma_{i}}\right]^{2},
$$

where $\sigma_{i}$ are the individual measurement errors. In an unlikely event that the measured data matches exactly the basis function set, $\chi^{2}=0$. Since this is impossible for experimentally obtained data due to the individual measurement errors, the $a_{k}$ are chosen such that $\chi^{2}$ is minimized.

There are several means of minimizing the merit function. The most general is through solution of normal equations. This is achieved by setting to zero the derivatives of Equation 5.14 with respect to each of the $M$ individual basis functions. The resulting $M$ equations are called the normal equations, which can be written in a matrix notation as

$$
\left(A^{T} \cdot A\right) \cdot a=A^{T} \cdot b,
$$

where $A$ is the design matrix of the fitting problem, $b$ is the measurement value vector (length $N$ ) and $a$ is a vector of coefficients $\left(a_{k}\right)$ to be determined by solution of Equation 5.15 (length $M$ ). The design matrix has dimension $N \times M$, with component elements $A_{i j}=\frac{X_{j}\left(x_{i}\right)}{\sigma_{i}}$ constructed from the $M$ basis functions evaluated at the $N$ abscissas $x_{i}$ and from the $N$ measurement errors $\sigma_{i}$.

Provided that the number of measurements is greater than or equal to the number of basis functions $(N \geq M)$ and there are no degeneracies, a solution for the 
inverse design matrix, $A^{-1}=A^{T}$ can be determined and applied in Equation 5.15 to find the coefficient solution vector $a$. For dual-energy decomposition, this is usually not a difficulty since the permutation number for iodine and plexiglas calibration steps used far exceeds the number of basis functions selected for the analysis. Calculation of the normal equation inverse design matrix can be achieved through Gauss-Jordan elimination or LU decomposition with backsubstitution [20].

Various decomposition methods differ according to what variables are used in the design matrix and measurement vector, upon what subset of the entire calibration data set is used in the minimization and how the eventual material equivalent images are calculated. The earliest developed techniques and those most customary today, use the entire calibration data set to yield a set of global basis function coefficients.

\subsection{Global Coefficient Algorithms}

Global coefficient algorithms include the non-linear ("indirect") equation technique of Alvarez and Macovski [3], and the direct approximation method enumerated by Nalcioglu [52]. As already elaborated in Section 5.2.2, assuming highly monochromatic high- and low-energy sources with sufficient flux, Equation 5.10 can be simplified to [18]:

$$
\begin{aligned}
& \ln \left(\frac{I^{H}}{I_{0}^{H}}\right)=-\mu_{p l}^{H} t_{p l}-\mu_{i o}^{H} t_{i o}, \\
& \ln \left(\frac{I^{L}}{I_{0}^{L}}\right)=-\mu_{p l}^{L} t_{p l}-\mu_{i o}^{L} t_{i o},
\end{aligned}
$$

where $I$ and $I_{0}$ are the transmitted and reference intensities at the specified energies, respectively. The solution to 5.18 for the thickness values of plexiglas and iodine, $t_{p l}$ and $t_{i o}$, is a simple linear combination of the natural logarithms of the transmitted intensities obtained at the two different X-ray energies:

$$
\left[\begin{array}{l}
\ln \left(\frac{I^{H}}{I_{0}^{H}}\right) \\
\ln \left(\frac{I^{L}}{I_{0}^{L}}\right)
\end{array}\right]=-\left[\begin{array}{cc}
\mu_{p l}^{H} & \mu_{i o}^{H} \\
\mu_{p l}^{L} & \mu_{i o}^{L}
\end{array}\right]\left[\begin{array}{c}
t_{p l} \\
t_{i o}
\end{array}\right] .
$$

The differential attenuation of the high- and low-energy portions of the polychromatic spectrum results in a nonlinear relationship between the logarithm of the X-ray intensities and equivalent thicknesses. Since Equation 5.17 can no longer be used, we empirically model this nonlinear relationship as

$$
\begin{aligned}
& T_{H}=\ln \left(\frac{I^{H}}{I_{0}^{H}}\right)=b_{0}+b_{1} t_{p l}+b_{2} t_{i o}+b_{3} t_{p l} t_{i o}+b_{4} t_{p l}^{2}+b_{5} t_{i o}^{2}+\ldots \\
& T_{L}=\ln \left(\frac{I^{L}}{I_{0}^{L}}\right)=c_{0}+c_{1} t_{p l}+c_{2} t_{i o}+c_{3} t_{p l} t_{i o}+c_{4} t_{p l}^{2}+c_{5} t_{i o}^{2}+\ldots
\end{aligned}
$$


The coefficient vector entries $b_{i}$ and $c_{i}$ are then determined through normal equation solution using plexiglas and iodine thickness polynomials as design matrix elements $\left(A_{i j}\right)$ and the high- and low-energy transmission calibration values in the measurement vector $(b)$. The final result, plexiglas and iodine equivalent basis images $\left(t_{p l}\right.$ and $t_{i o}$ ) must then be estimated by simultaneous solution of Equation 5.18 with $T_{H}$ and $T_{L}$ values from corresponding mean pixel values (ROIs) in the high- and lowenergy images. A solution is typically achieved iteratively using the Newton-Raphson method, with the linear terms providing an initial guess [20].

Several limitations of this decomposition algorithm are well recognized. Firstly, calculation of the inverse design matrix by Gauss-Jordan elimination or LU decomposition can produce unsatisfactory results if the original set of equations are illconditioned. This is typically the case when the spectral distinctness between highand low-energy images is limited. The second drawback is the indirect calculation of the basis image thicknesses $t_{p l}$ and $t_{i o}$ from the transmission data. The NewtonRaphson method has poor global convergence properties, so that the initial guess at the root has to be somewhat near the actual root for convergence [42], although bisectioning techniques can cure this problem to a limited extend.

The "direct" approximation method attempts to remedy the shortcoming of the non-linear algorithm through expression of $t_{p l}$ and $t_{i o}$ by polynomials in $T_{H}$ and $T_{L}$, following recasting Equation 5.17:

$$
\begin{gathered}
{\left[\begin{array}{c}
t_{p l} \\
t_{i o}
\end{array}\right]=-\left[\begin{array}{ll}
\mu_{p l}^{H} & \mu_{i o}^{H} \\
\mu_{p l}^{L} & \mu_{i o}^{L}
\end{array}\right]^{-1}\left[\begin{array}{c}
\ln \left(\frac{I^{H}}{I_{0}^{H}}\right) \\
\ln \left(\frac{I^{L}}{I_{0}^{L}}\right)
\end{array}\right]} \\
t_{p l}=d_{0}+d_{1} T_{H}+d_{2} T_{L}+d_{3} T_{H} T_{L}+d_{4} T_{H}^{2}+d_{5} T_{L}^{2}+\ldots, \\
t_{i o}=e_{0}+e_{1} T_{H}+e_{2} T_{L}+e_{3} T_{H} T_{L}+e_{4} T_{H}^{2}+e_{5} T_{L}^{2}+\ldots
\end{gathered}
$$

The coefficient vector entries $d_{i}$ and $e_{i}$ are then determined through normal equation solution using the high- and low-energy transmission calibration polynomials as design matrix elements and the associated plexiglas and iodine thicknesses as measurement vector entries. Subsequent to coefficient determination, the plexiglas and iodine equivalent thicknesses are easily calculated directly, point by point from Equation 5.20 using corresponding high- and low-energy transmission values. Thus, the Newton-Raphson process is eschewed, while the problems associated with illconditioned linear equations are not. Consequently, the condition of choosing two very distinct spectra with no (or minimum) overlaps must be a priority.

Both of the above global coefficient algorithms were elaborated upon by Lehmann [43] who termed them the "iterative fit" and "closed form fit", respectively. He also added a "weighted closed form fit" in order to produce smaller estimation error residuals where the quantum noise in $T_{H}$ and $T_{L}$ is less severe. These algorithms utilize the entire calibration data set to solve for the least squares coefficient vector. 


\subsection{Test Phantom}

Decomposition coefficients obtained in the calibration method are used to separate soft tissue and iodine components of a home-made test mouse phantom. The phantom is similar to a microtitration multi-well plate, made of a 1.48-cm-thick plexiglas block, and consisting of 48 wells, arranged as a $8 \times 6$ matrix (Figure 5.3). The wells all have flat bottoms except the last row containing hemisphere-bottom wells. In each row, the wells have the same depth, and the depth increases from row 1 through row 7 as 1, 2, 3, 4, 5, 7, and $10 \mathrm{~mm}$, respectively. Each column includes wells with the same diameter varying as 3, 4, 5, 6, 7, and $10 \mathrm{~mm}$, respectively. A 2-cm gap is provided between adjacent wells in each row and column to limit possible wells scatter communications. The depth of wells is chosen such that it covers the same range of iodine projection thicknesses used in the calibration phantom. Besides, slabs of catfish fillets containing less than $6 \%$ fat are placed on top of the multi-well plate to better produce soft tissue variation in the images. The engineering drawing of the test phantom is shown in Appendix B (Test Phantom 2).

\subsection{Computer Model}

\subsubsection{Signal-to-Noise Ratio (SNR) in the Subtracted Iodine Image}

Lemacks et al. [44] presented a theoretical framework to calculate SNR in a subtracted dual-energy image. Here, their formalism is extended for dual-energy subtraction using appropriate filters for high- and low-energy spectra and applied to the subtracted iodine image.

The mean measured signal per unit tube loading, $S_{i}$, and its noise variance, $\sigma_{i}^{2}$, for an area on the image, $a\left(\mathrm{~mm}^{2}\right)$, obtained using filtered high- and low-energy spectra can be expressed as

$$
\begin{aligned}
S_{i} & =a \int \phi_{i}(E) e^{-\mu_{p l}(E) t_{p l}-\mu_{i o}(E) t_{i o}} A(E) Q(E) d E, \\
\sigma_{i}^{2} & =a \int \phi_{i}(E) e^{-\mu_{p l}(E) t_{p l}-\mu_{i o}(E) t_{i o}} A(E) Q^{2}(E) d E,
\end{aligned}
$$

where $\phi_{i}(E)$ is the filtered photon flux per unit tube loading per unit energy $\left(\frac{\text { photons }}{m m^{2} k e V m A s}\right)$ at the detector input without object (iodine and plexiglas) for highor low-energy spectrum $(i=h, l) ; A(E)$ is the photon absorption ratio of the detector as a function of $E$; and $Q(E)(\approx E)$ is the detector response function. $\mu_{p l}(E)$, $\mu_{i o}(E), t_{p l}$ and $t_{i o}$ are the linear attenuation coefficients of iodine and plexiglas with their corresponding thicknesses, respectively. 
For a given signal, the image signal-to-noise ratios in the subtracted images are determined by the variances of the subtracted image noise. When dual-energy images are subtracted to eliminate the soft tissue, the variance in the energy-subtracted signals for iodine, $\sigma_{i o}^{2}$, becomes

$$
\sigma_{i o}^{2}=\frac{k_{i o, h}^{2}}{S N R_{h}^{2}}+\frac{k_{i o, l}^{2}}{S N R_{l}^{2}},
$$

where

$$
\begin{gathered}
S N R_{h}=\frac{S_{h}}{\sigma_{h}}, \\
S N R_{l}=\frac{S_{l}}{\sigma_{l}}, \\
k_{i o, h}=\frac{\overline{\mu_{p l, l}}}{\overline{\mu_{p l, l} \mu_{i o, h}}-\overline{\mu_{p l, h} \mu_{i o, l}}}, \\
k_{i o, l}=\frac{-\overline{\mu_{p l, h}}}{\overline{\mu_{p l, l} \mu_{i o, h}}-\overline{\mu_{p l, h} \mu_{i o, l}}},
\end{gathered}
$$

$\overline{\mu_{m, n}}(m=p l, i o ; n=h, l)$ is the effective attenuation coefficient averaged over the detected high- and low-energy spectra. The resultant SNR in the subtracted iodine image, $S N R_{i o}$, is then given as [44]

$$
S N R_{i o} \equiv \frac{t_{i o}}{\sigma_{i o}}=\frac{t_{i o}}{\sqrt{\frac{k_{i o, h}^{2}}{S N R_{h}^{2}}+\frac{k_{i o, l}^{2}}{S N R_{l}^{2}}}} .
$$

The soft tissue structures are canceled out in the iodine image, providing a uniform background signal.

\subsubsection{Dose}

Monte Carlo simulations were performed using previously validated SIERRA code [11]. The mouse was modeled as a 3 -cm tissue-equivalent (plexiglas) cylinder with density of $1 \mathrm{gr} / \mathrm{cm}^{3}$. The X-ray energy imparted throughout the animal's tissue (except in the dermal layer) was tallied and included in the dose computation, including X-ray energy scattered "outside" the tissue volume. A 1-mm-thick layer of dermis and fur was also modeled, and X-ray energy deposition interior to the dermal/fur layer was tallied. The fur and metabolically inactive dermis are relatively insensitive to the effects of ionization, and the dose of interest to the mouse is the one deposited in the internal organs. In conclusion, the radiation dose to the mouse was estimated as

$$
D_{p}(d, k V p)=k \sum_{0}^{k V p} \phi(E) D_{m}(d, E)
$$


where $\phi(E)$ is the normalized poly-energetic X-ray spectrum, $d$ is the mouse thickness $(=3 \mathrm{~cm}), D_{m}(d, E)$ is the monoenergetic dose, $D_{p}(d, k V p)$ is the polyenergetic dose, and $k$ is a normalization constant. $D_{p}(d, k V p)$ is in the units of absorbed tissue dose per air kerma and is used to convert measured air kerma to mean absorbed dose in the animal. 


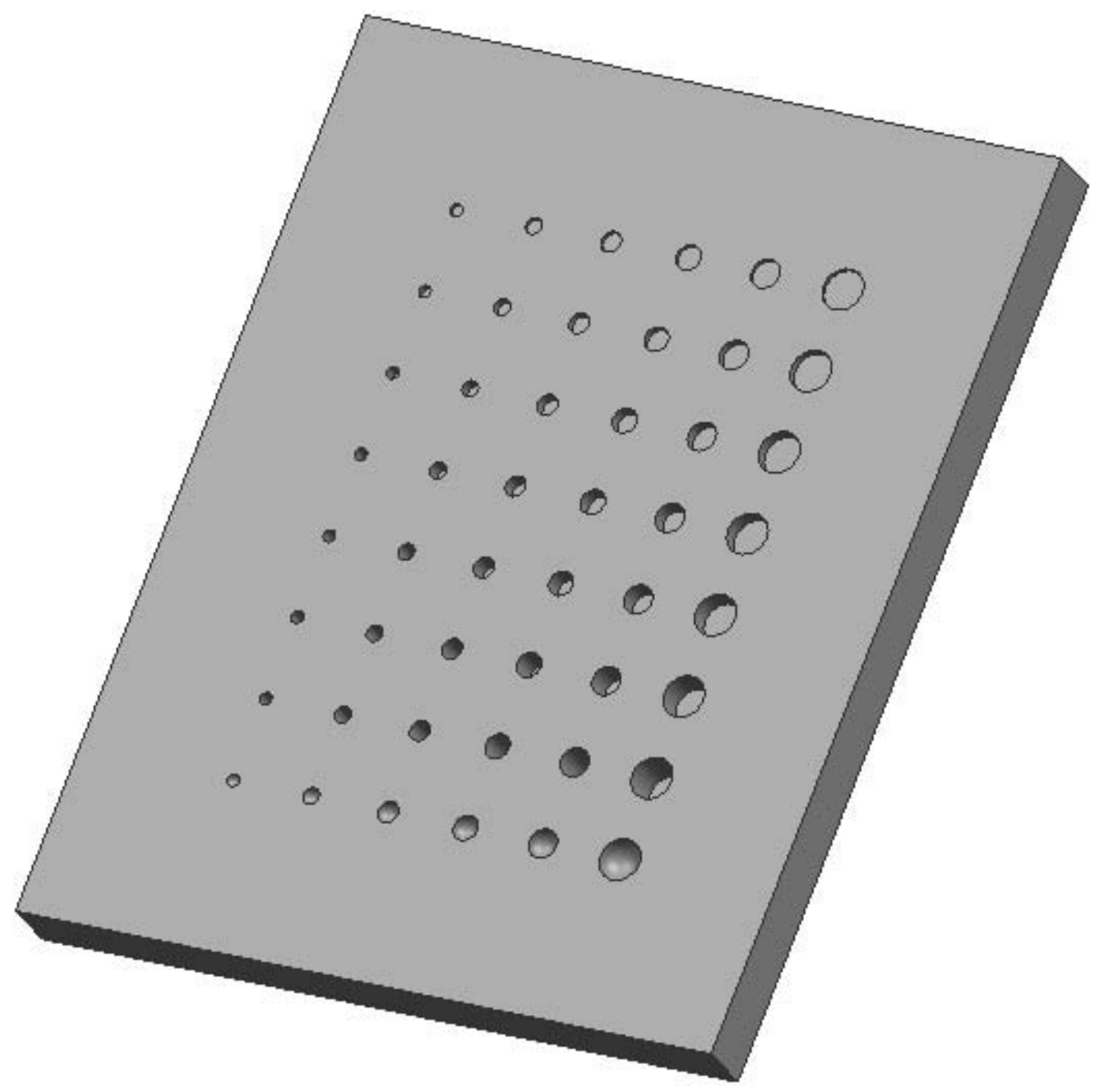

Figure 5.3: Test phantom. 


\section{Chapter 6}

\section{Temporal Subtraction Technique}

\subsection{Introduction}

Time-dependent or temporal subtraction technique constitutes the underlying physics of Digital Subtraction Angiography (DSA) in which the non-invasive study of vessels is the principal motivation. In the early days of DSA, in an invasive procedure, a catheter was inserted into an artery and large amount of iodinated contrast agent was injected, resulting in good vessel visualization despite the intervening anatomical structures [48]. In a non-invasive and safer routine in which low concentration of iodinated contrast agent was injected intravenously, subtraction technique was employed to eliminate the intervening tissue $[48,65]$. However, iodine visualization using intravenous injections is limited by overlapping iodinated arteries since the IV bolus arrives in most arteries in a field of view at roughly the same time. Intravenous injections are rarely, if ever, still used today. Improved sensitivity of DSA equipment allowed the amount of iodine required to produce good images using arterial injections to be significantly reduced. Thus, arterial injections are no longer considered to be an invasive procedure. Direct arterial injections have the advantage over venous injections in isolating the artery of interest without the background noise due to other overlapping iodinated arteries.

The temporal subtraction technique involves obtaining images before and shortly after administering the contrast agent. Then, the pre-contrast (mask) image is logarithmically subtracted from the post-contrast images to enhance the contrast of iodinated region and suppress the background. The simplest form of temporal subtraction uses a single mask and a post-contrast image. The next level of sophistication averages several mask images and/or several post-contrast images prior to subtraction since averaging can reduce image noise or motion artifacts [15]. The kinetics of the contrast agent and blood flow can also be assessed using temporal subtraction technique.

Functional imaging in small animal models can be addressed well using DSA. Scaling DSA to the higher spatial and temporal resolutions, optimization of radiographic spectra to produce the best contrast and signal-to-noise ratio, and particularly acquiring images synchronous to biological rhythms, such as cardiac or ventilatory cycles present challenges in employing DSA technique in small animals imaging [46]. Iodinated contrast agents designed for clinical studies can be used for imaging small animals, but they are cleared from the blood stream within seconds after intravenous 
injections due to the higher heart rate of rodents. Alternatively, a new class of bloodpool contrast agents has been developed whose molecules size is larger than that of capillary fenestration and phagocytosis by the reticuloendothelial system (RES) is thus limited by the chemical design such as pegylation [9]. The Fenestra ${ }^{\text {TM }}$ line of contrast agents (ART Advanced Research Technologies Inc., Saint-Laurent, Quebec, Canada), containing $50 \mathrm{mg} / \mathrm{cm}^{3}$ of iodine, provide long-lasting (few hours) and stable contrast enhancement for micro-CT imaging applications, thereby affording an opportunity to select optimal post-injection time points for imaging different organs [31]. In general, tail-vein injection is a common method of introducing such contrast agents.

\subsection{Theory}

Let $\mu_{w}(x, y, z)$ and $\mu_{i o}(x, y, z)$ be the total linear attenuation coefficients of tissue (water) in the anatomical region under study and administered iodinated contrast agent (iodine), respectively. The linear subtraction operation involves subtracting the mask and post-contrast images. Assuming a highly monoenergetic spectrum and no scattered radiation in the projection radiography:

$$
\begin{gathered}
I_{m}=I_{0} e^{-\mu_{w} t_{w}}, \\
I_{p c}=I_{0} e^{-\mu_{w} t_{w}-\mu_{i o} t_{i o}}, \\
\ln \left(\frac{I_{p c}}{I_{0}}\right)-\ln \left(\frac{I_{m}}{I_{0}}\right)=-\mu_{i o} t_{i o},
\end{gathered}
$$

where $I_{m}, I_{p c}$, and $I_{0}$ are the transmitted mask, transmitted post-contrast, and reference monoenergetic intensities, respectively. $t_{w}$ and $t_{i o}$ are soft tissue and contrast agent thicknesses, respectively, with their corresponding total linear attenuation coefficients $\mu_{w}$ and $\mu_{i o}$. The subtraction result is essentially linear with the amount of iodine in the path of spectrum.

The quality of subtracted iodine image is limited by noise and motion artifacts due to various physiological motions occurring between acquisition of mask and postcontrast images. This effect can be minimized by storing a number of mask and/or post-contrast images and finding a pair that provides acceptable tissue subtraction performance. In clinical studies where the imaged anatomy is relatively static, such as carotid arteries, excellent images have been produced. The subtraction operation is made highly stable using digital fluoroscopy in which the output of the digital video camera is stored and appropriately processed to provide the subtracted image [48]. 


\subsection{Test Phantom}

The same test phantom described in Section 5.5 is used to evaluate the performance of temporal subtraction technique in enhancing the contrast of iodinated wells and suppressing the background. The multi-well plate provides a post-contrast image. A 1.48-cm-thick flat plexiglas block having the same dimensions as the multiwell plate is used to supply the mask image (Figure 6.1). Similar to the dual-energy subtraction technique, slabs of catfish fillets produce soft tissue variations in the images. The engineering drawing of the mask phantom is shown in Appendix B (Test Phantom 1).

\subsection{Computer Model}

\subsubsection{Signal Difference-to-Noise Ratio (SdNR)}

The mask and post-contrast signals are theoretically calculated using Equation 5.21. The SdNR is then expressed as

$$
S d N R=\frac{S_{p c}-S_{m}}{\sqrt{\sigma_{p c}^{2}+\sigma_{m}^{2}}},
$$

where $S_{p c}$ and $S_{m}$ are the detected mask and post-contrast signals, respectively, with their corresponding variances, $\sigma_{p c}^{2}$ and $\sigma_{m}^{2}$, calculated by Equation 5.22.

\subsubsection{Dose}

The absorbed radiation dose is estimated using the same model framework explained in Section 5.6.2. 


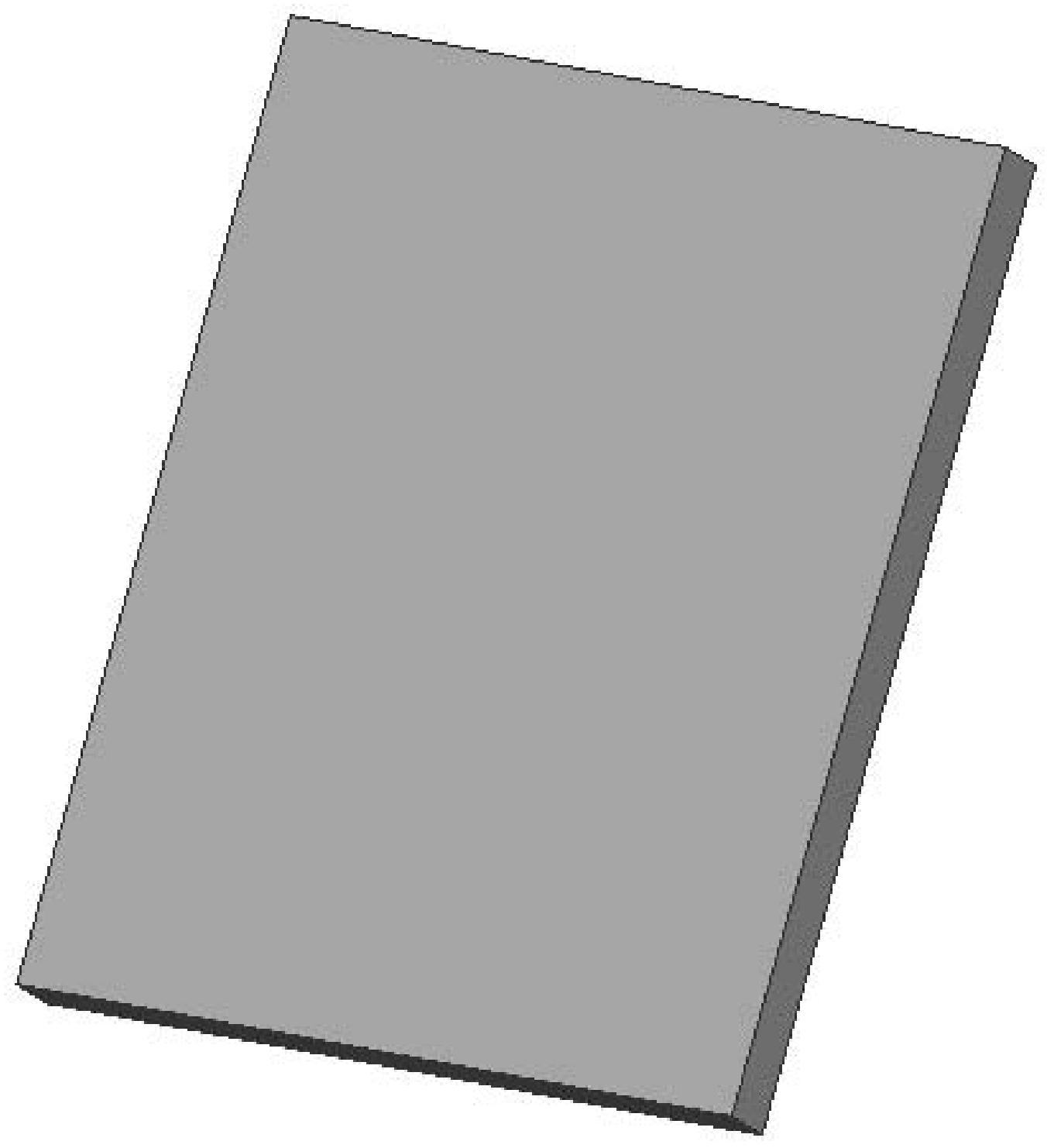

Figure 6.1: Test phantom (mask). 


\section{Chapter 7}

\section{Results and Discussion}

\subsection{Image Quality Assessment of CR 7400}

\subsubsection{Exposure Geometry}

Figure 7.1 shows a plot of the maximum flux as a function of focal spot size calculated for two limiting CR resolutions of 50 and 100 microns, using Equation 4.3 in Section 4.3. The object-to-detector distance was set to $22 \mathrm{~cm}$, ensuring a rather conservative gap between the phantom and detector. The current X-ray tube has 0.6 and $1.2 \mathrm{~mm}$ focal spots. For $0.6 \mathrm{~mm}$ focal spot, the source-to-detector values for $50-$ and 100-micron resolutions are 286 and $154 \mathrm{~cm}$, respectively. For $1.2 \mathrm{~mm}$ focal spot and corresponding resolutions of 50 and 100 microns, source-to-detector distances are 550 and $286 \mathrm{~cm}$, respectively. Our choice of imaging with the current experimental set-up in all phases of the project was the geometry for 0.6-mm focal spot at 100-micron resolution, with source-to-detector and object-to-detector distances of 154 and $22 \mathrm{~cm}$, respectively, providing maximum flux and causing a small magnification $(\sim 1.16)$.

\subsubsection{Response Curve}

Figure 7.2 illustrates the response curve of the system measured as described in Section 4.4. It presents a linear response at all exposures with correlation coefficient for linear regression fit of 0.999. At each exposure, which was repeated five times, the average pixel value variation for the extracted ROIs was within $6 \%$, as illustrated by small error bars.

\subsubsection{Presampled MTF}

Figure 7.3 shows ten extracted ROIs at 100-micron resolution. Among ROIs at each resolution, ESF and LSF of ROI \#5 at 100-micron resolution are shown in Figures 7.4, (a) and (b). Figure 7.5 reports the measured presampled MTFs in scan direction for 50- and 100-micron resolutions, respectively. A sixth-degree polynomial 


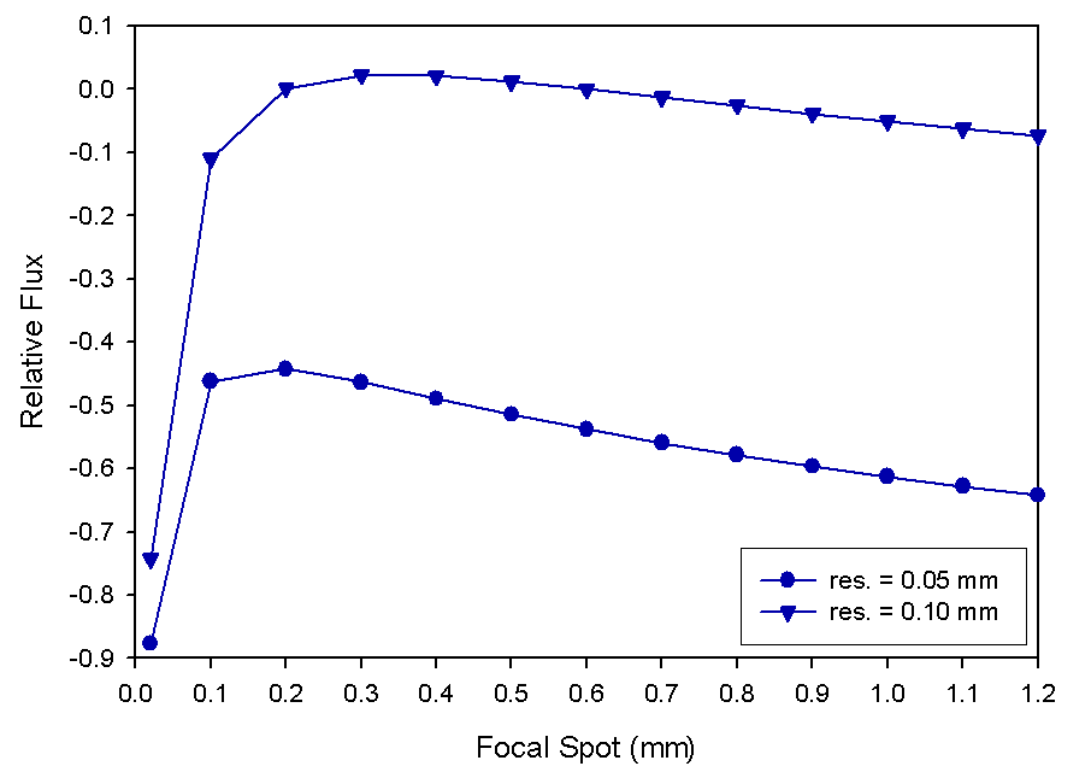

Figure 7.1: Log scale of relative flux as a function of focal spot size.

The flux is normalized to the maximum attained for resolution of 100 microns and focal spot size of $0.6 \mathrm{~mm}$.

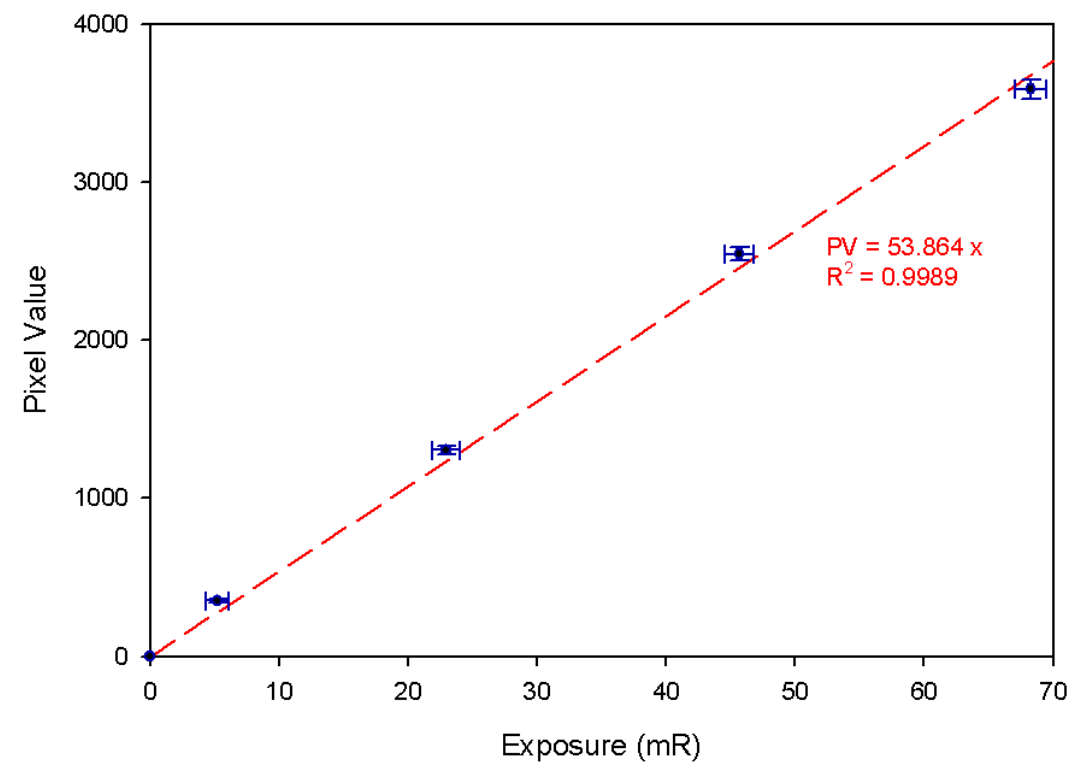

Figure 7.2: Transfer response characteristics of CR 7400.

The exposure error bar variation was about $16 \%$ for $5.22 \mathrm{mR}$, and within $6 \%$ for all other exposures. The PV error bar variation was within $3 \%$ at all studied exposures. 


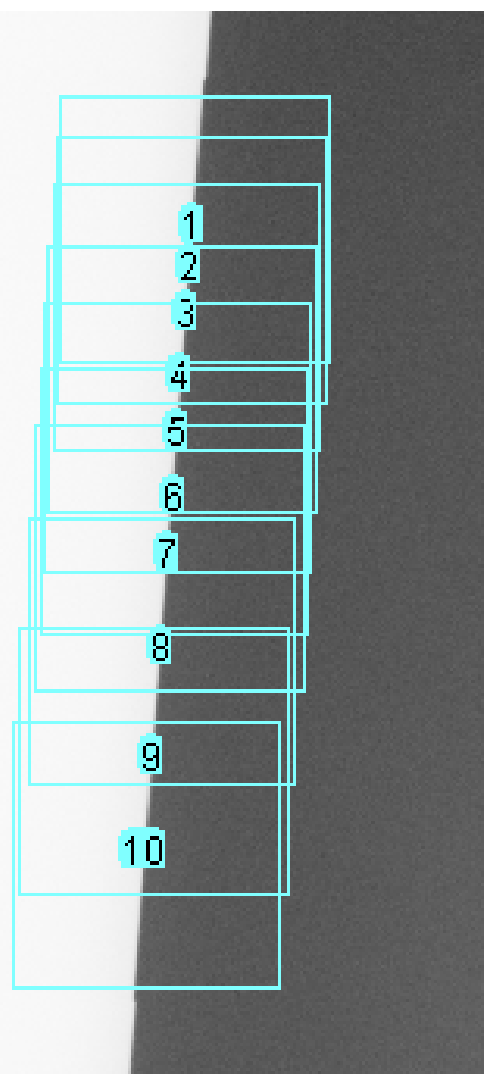

Figure 7.3: Extracted ROIs for MTF calculation. 


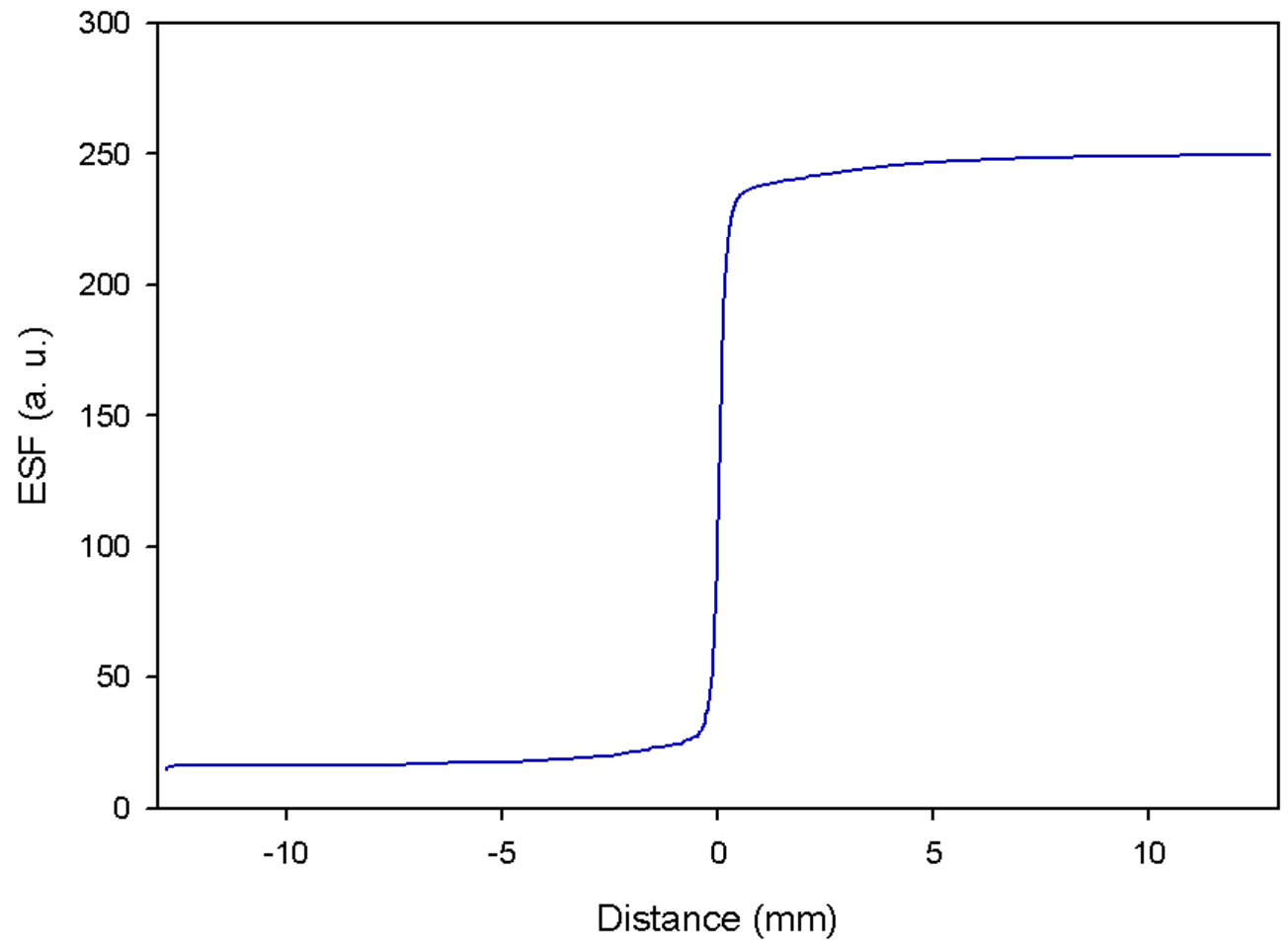

(a)

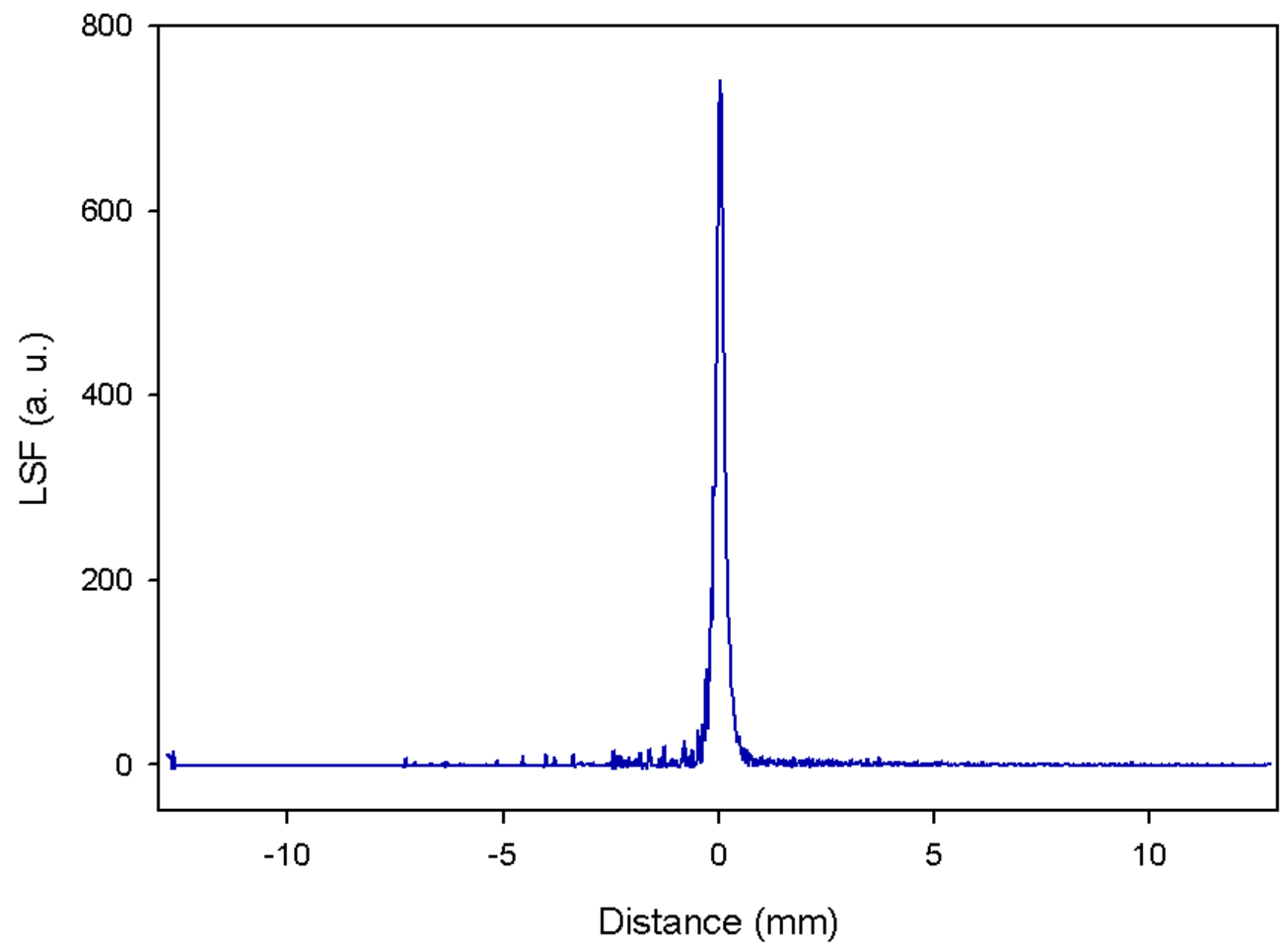

(b)

Figure 7.4: ROI \#5 at 100-micron resolution: (a) ESF. (b) LSF. 


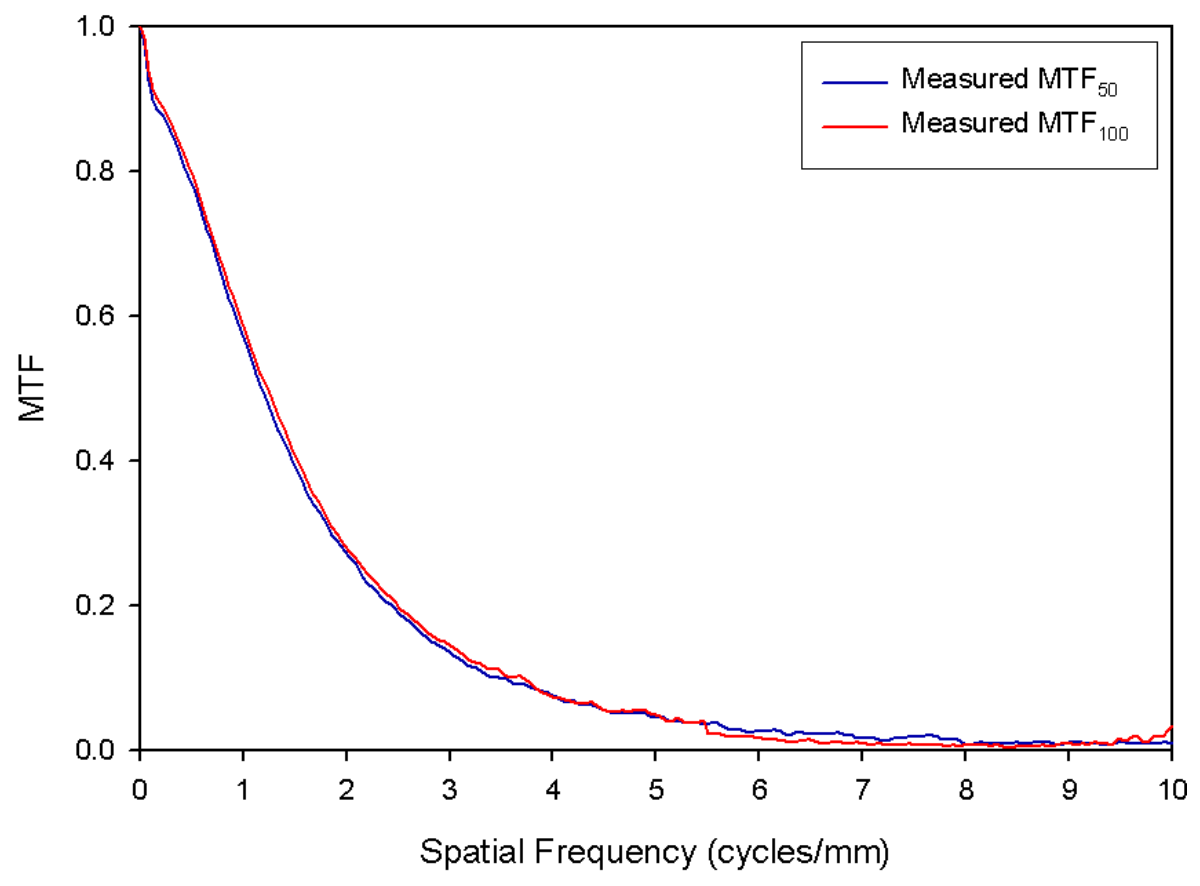

(a)

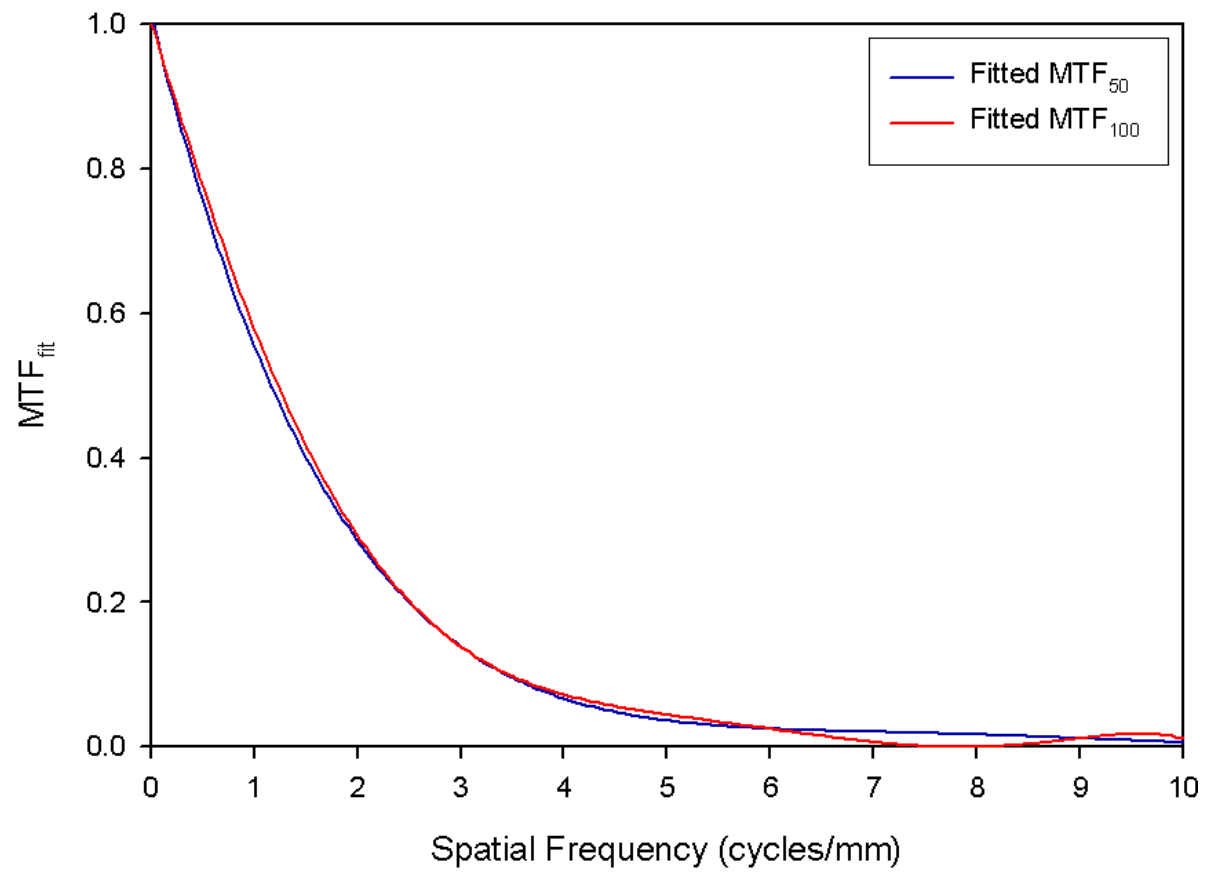

(b)

Figure 7.5: Measured MTFs and their corresponding fits.

(a) The presampled measured MTFs at 50-micron and 100-micron resolutions, displayed up to $10^{\text {cycles }} / \mathrm{mm}$. Not shown are MTF error bars showing $9 \%$ and $5 \%$ variations for 50-micron and 100-micron resolutions, respectively. (b) The sixth-order polynomial MTF fits at 50-micron and 100-micron resolutions. 
Table 7.1: Parameters for the MTF sixth-degree polynomial fit $\left(\mathrm{y}=\mathrm{ax}^{6}+\mathrm{bx}^{5}+\mathrm{cx}^{4}+\mathrm{dx}^{3}+\mathrm{ex}^{2}+\mathrm{fx}+\mathrm{g}\right)$ at 50- and 100-micron resolutions $\left(\mathbf{R}^{2}=0.999\right)$.

\begin{tabular}{ccc}
\hline Parameter & $\mathrm{MTF}_{50}$ & $\mathrm{MTF}_{100}$ \\
\hline $\mathrm{a}$ & $(5.30 \pm 0.17) \times 10^{-7}$ & $(-1.92 \pm 0.17) \times 10^{-5}$ \\
$\mathrm{~b}$ & $(-3.88 \pm 0.10) \times 10^{-5}$ & $(5.76 \pm 0.52) \times 10^{-4}$ \\
$\mathrm{c}$ & $(1.14 \pm 0.02) \times 10^{-3}$ & $(-6.09 \pm 0.59) \times 10^{-3}$ \\
$\mathrm{~d}$ & $(-1.72 \pm 0.02) \times 10^{-2}$ & $(2.27 \pm 0.33) \times 10^{-2}$ \\
e & $(1.39 \pm 0.01) \times 10^{-1}$ & $(3.64 \pm 0.87) \times 10^{-2}$ \\
f & $(-5.84 \pm 0.03) \times 10^{-1}$ & $(-4.80 \pm 0.10) \times 10^{-1}$ \\
g & 1.02 & 1.01 \\
\hline
\end{tabular}

Notes: There was no significant statistical difference between the measured $\mathrm{MTF}_{50}$ and $\mathrm{MTF}_{100}(p-$ value $=0.92)$.

fit to the final MTF is also included in the MTF display with fitted parameters shown in Table 7.1. For both resolutions, no appreciable differences in scan and subscan MTFs $\left(\frac{M T F_{s c a n}-M T F_{\text {subscan }}}{M T F_{\text {scan }}} \times 100\right)$ are observed, as illustrated in Figure 7.6. Overall, there is little or no variation in the MTF as a function of pixel size, because presampled MTF, as measured, does not fundamentally depend on pixel size, as the effect of sampling is removed by the over-sampling methodology of the measurement. As already discussed, laser light scattering in the phosphor layer is the main limiting factor for CR resolution and changes in pixel size do not alter the spread of the laser light, and thus the presampled MTF.

\subsubsection{NPS}

Figure 7.7 illustrates the one-dimensional NPS in the horizontal (long-axis), vertical (short-axis), and diagonal directions. The shape of the NPS curves decrease gradually with increasing spatial frequency. As expected, the magnitude of NPS changes inversely with exposure. The shapes of the NPS curves are relatively independent of exposure, but tend to have higher slopes at higher exposures, possibly due to less added electronic noise. At each exposure, the magnitude of the NPS values have small differences in each direction. The NPS in the horizontal and vertical directions are similar at low spatial frequencies, but as the frequency increases, the horizontal NPS drops to lower values than that in the vertical direction, indicating less noise at mid-high spatial frequencies in the horizontal direction. This effect can be explained by the time the laser light takes to be adjusted on the next scanning line during which some high-frequency noise is introduced. The difference between the NPS in the horizontal and vertical directions corresponds closely to the small differences observed in the scan and subscan MTFs. 


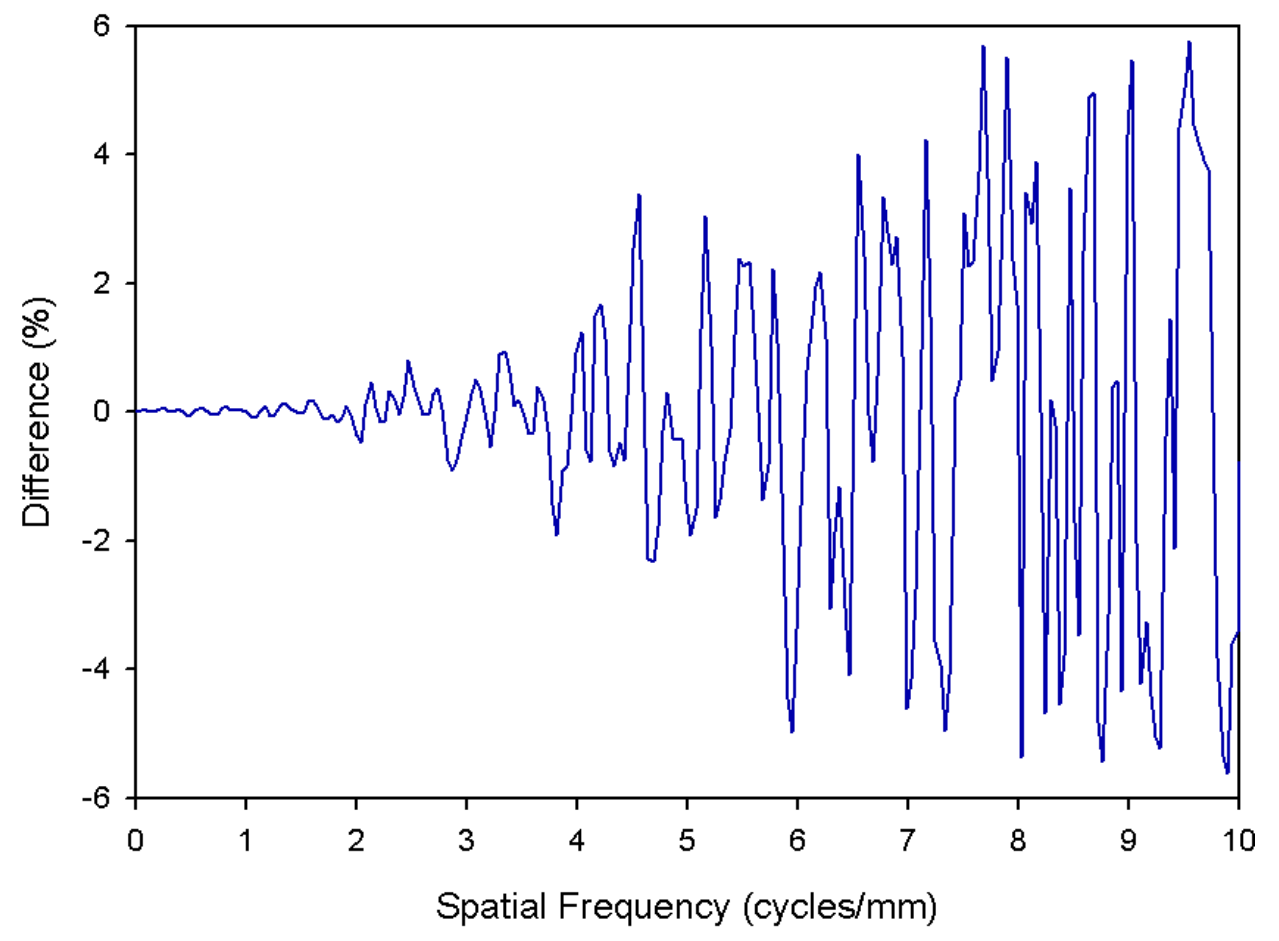

(a)

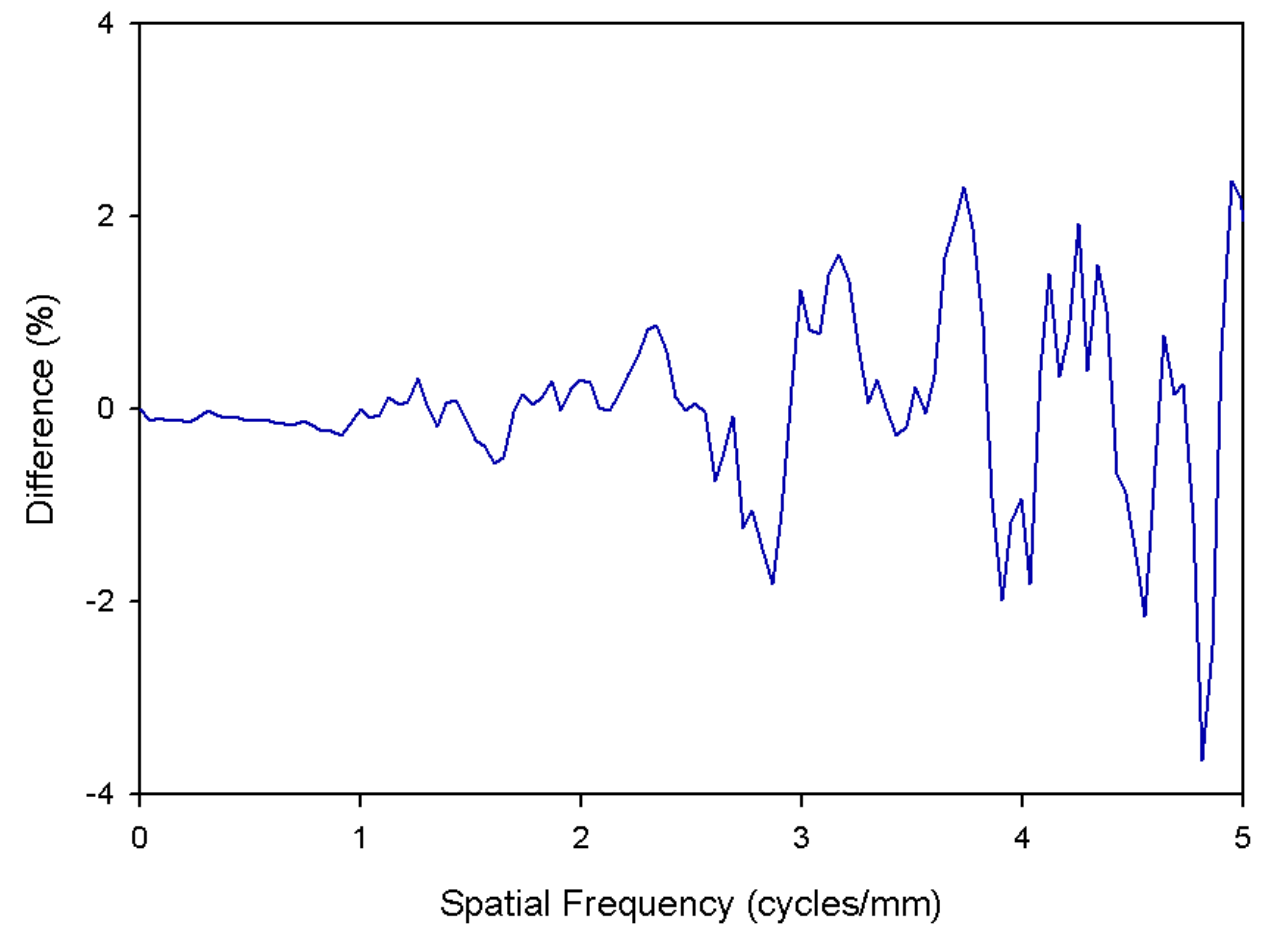

(b)

Figure 7.6: The difference in scan and subscan MTFs $\left(\frac{\mathrm{MTF}_{\text {scan }}-\mathrm{MTF}_{\text {subscan }}}{\mathrm{MTF}_{\text {scan }}} \times 100\right)$ for (a) 50-micron and (b) 100-micron resolutions, displayed up to the Nyquist frequencies. 


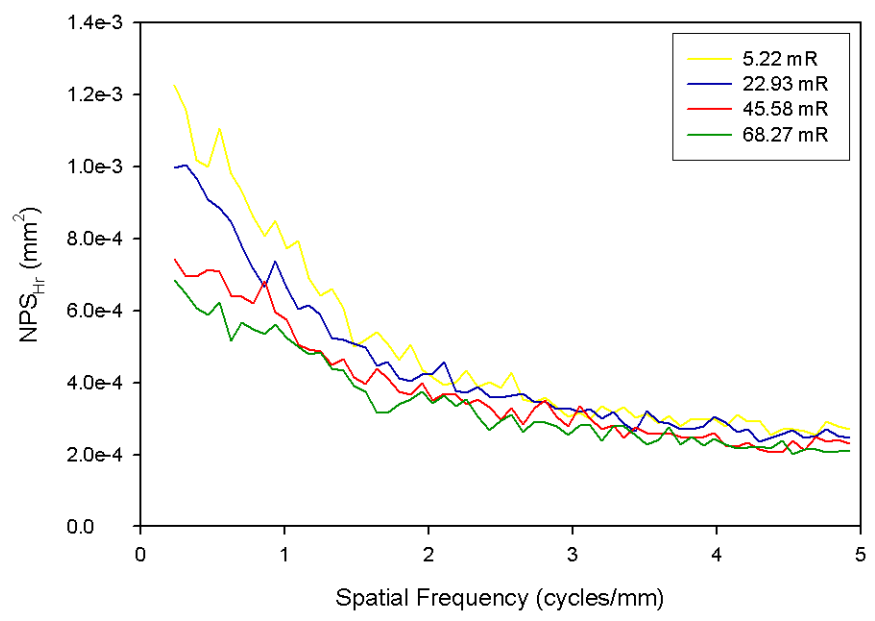

(a)

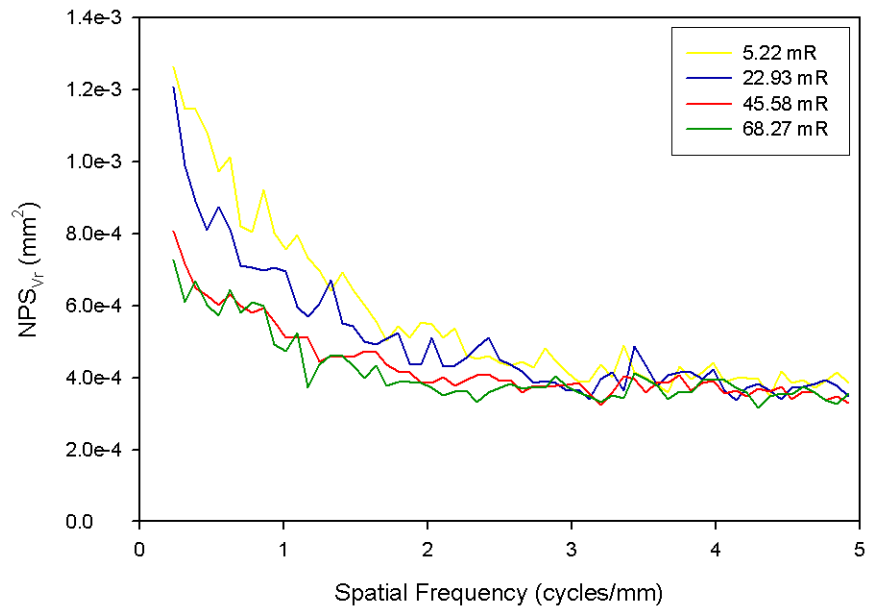

(b)

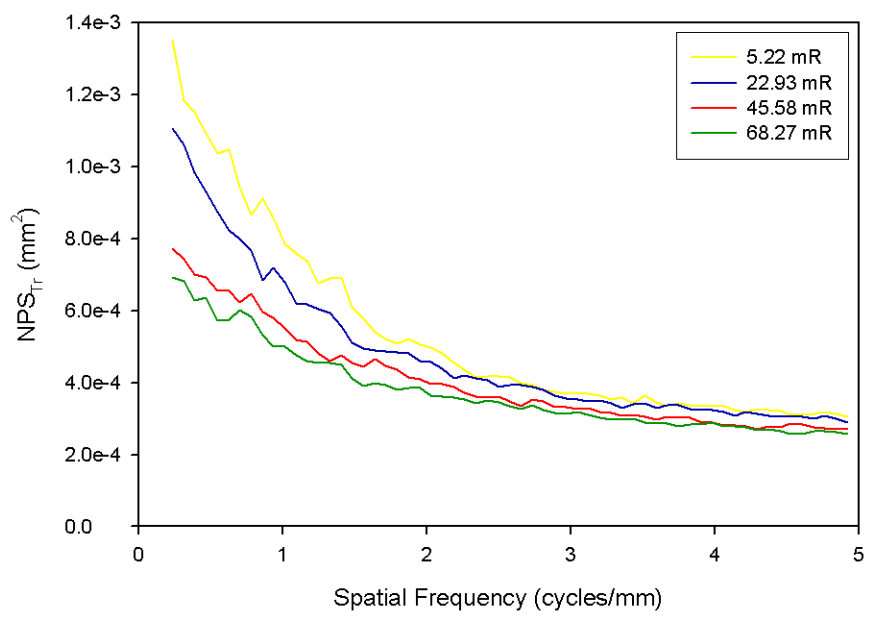

(c)

Figure 7.7: The 1D NPS in (a) horizontal, (b) vertical, and (c) diagonal directions.

Not shown are error bars showing $6 \% \sim 9 \%$ variation at the frequencies displayed. 
Table 7.2: RSD analysis: values of the main noise components for CR $7400\left(R^{2}=0.961\right)$.

\begin{tabular}{cc}
\hline Component & CR 7400 \\
\hline Poisson: $\alpha$ & $(1.96 \pm 0.13) \times 10^{-3}$ \\
Multiplicative: $\beta$ & $(2.78 \pm 0.63) \times 10^{-4}$ \\
Additive: $\gamma$ & $(2.62 \pm 0.40) \times 10^{-7}$ \\
\hline
\end{tabular}

\subsubsection{RSD}

Table 7.2 shows the values of main noise components for CR 7400 as estimated by the RSD analysis in Equation 4.6. The RSD analysis shows that the statistical noise is the most dominant noise component and the electronic noise, mainly contributed by PMTs is negligible. Consequently, CR 7400 seems to be quantum noise limited at the clinical exposures studied.

\subsubsection{NEQ}

Figure 7.8 shows NEQ in the horizontal and vertical directions. As shown in Equation 4.8., NEQ is a function of MTF and NPS. Since MTF is relatively insensitive to the exposure, NEQ behavior is solely determined by NPS, and increases with exposure.

\subsubsection{DQE}

Figure 7.9 illustrates the DQE in the horizontal and vertical directions. The DQE decreases with increased exposure. At each exposure, little difference is observed between the horizontal and vertical DQEs, as the directional dependencies of the MTF and NPS (Sections 7.1.3 and 7.1.4) cancel each other when the DQE is deduced.

\subsection{Dual-energy Subtraction Technique}

\subsubsection{Spectra}

Figure 7.10 illustrates filtered high- and low-energy spectra used in dual-energy subtraction technique considering exposure to $1-\mathrm{mm}^{2}$ area. The challenge was to create X-ray spectra containing the highest possible proportion of X-ray photons with energies above the K-edge, while maintaining an adequate flux and acceptable 


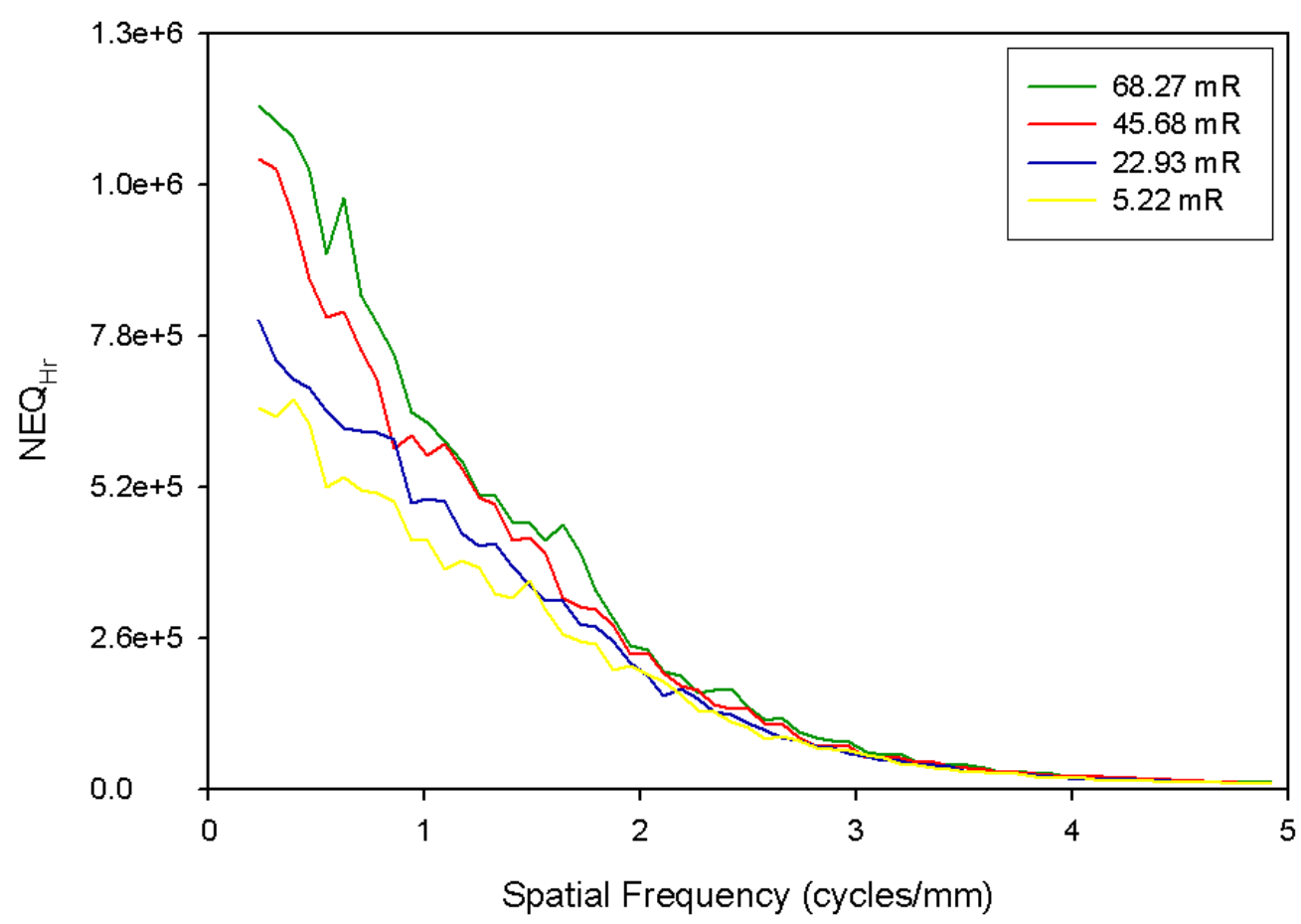

(a)

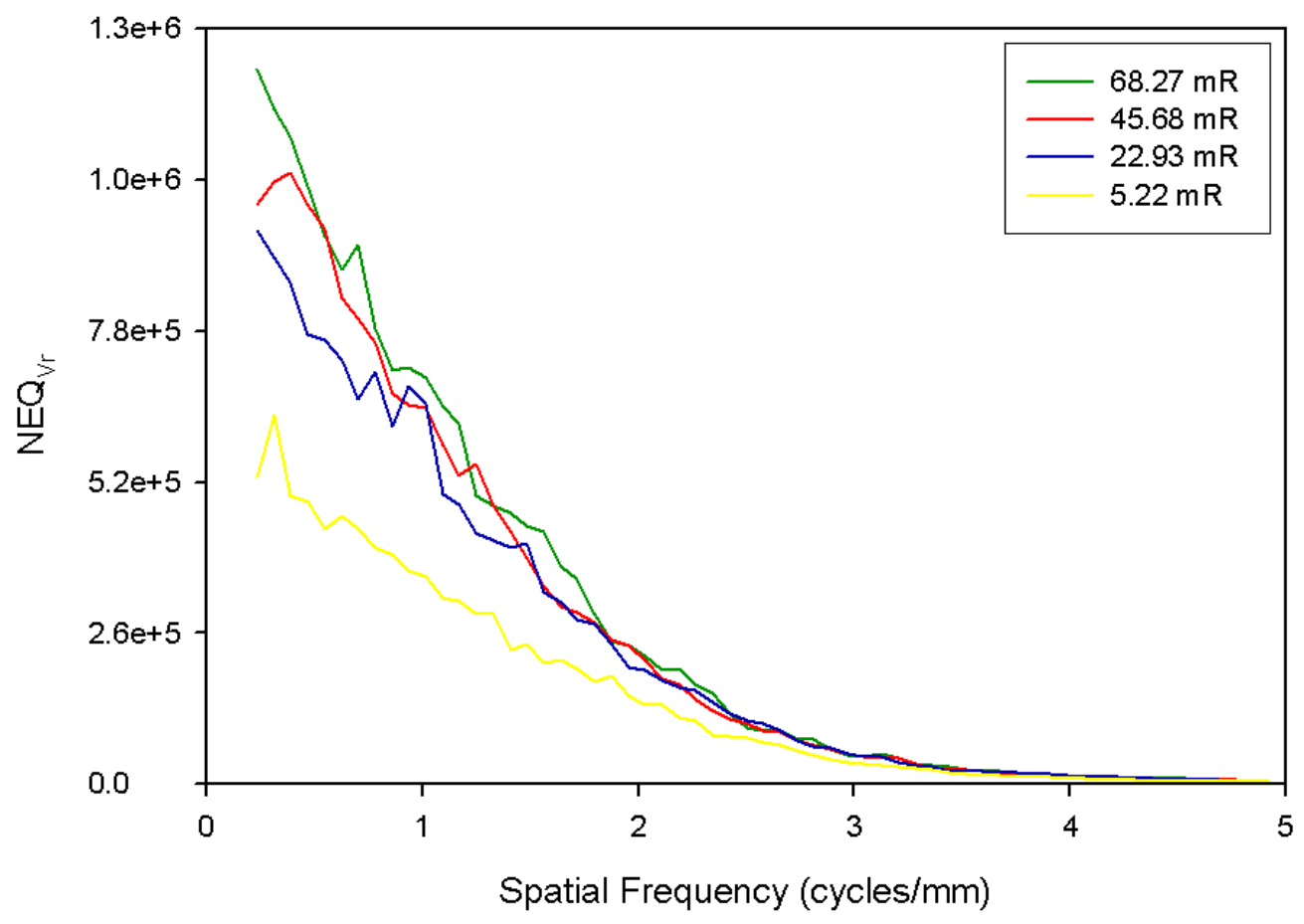

(b)

Figure 7.8: The NEQ in (a) horizontal and (b) vertical directions.

Not shown are error bars showing $7 \% \sim 10 \%$ variation at the frequencies displayed. 


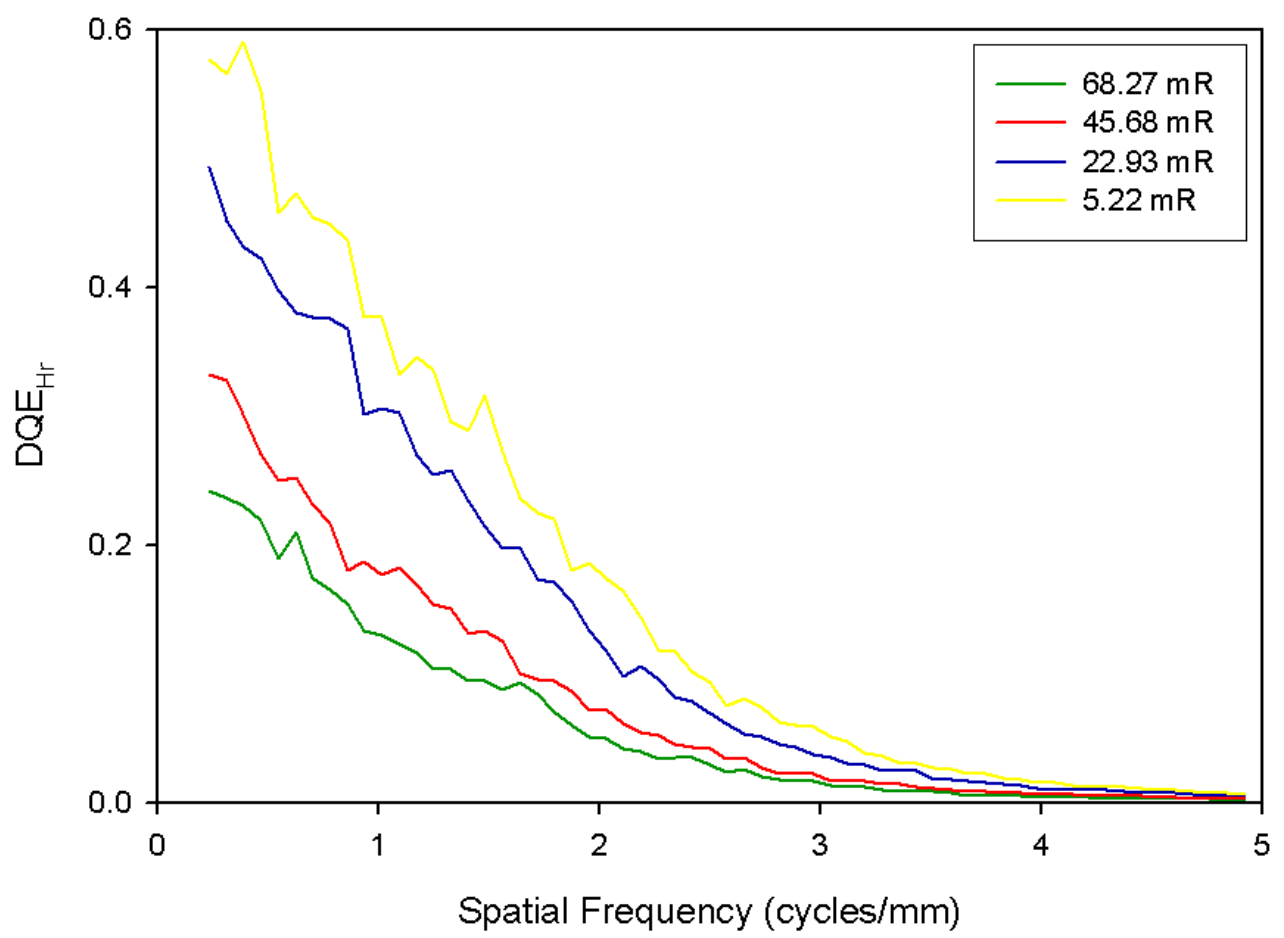

(a)

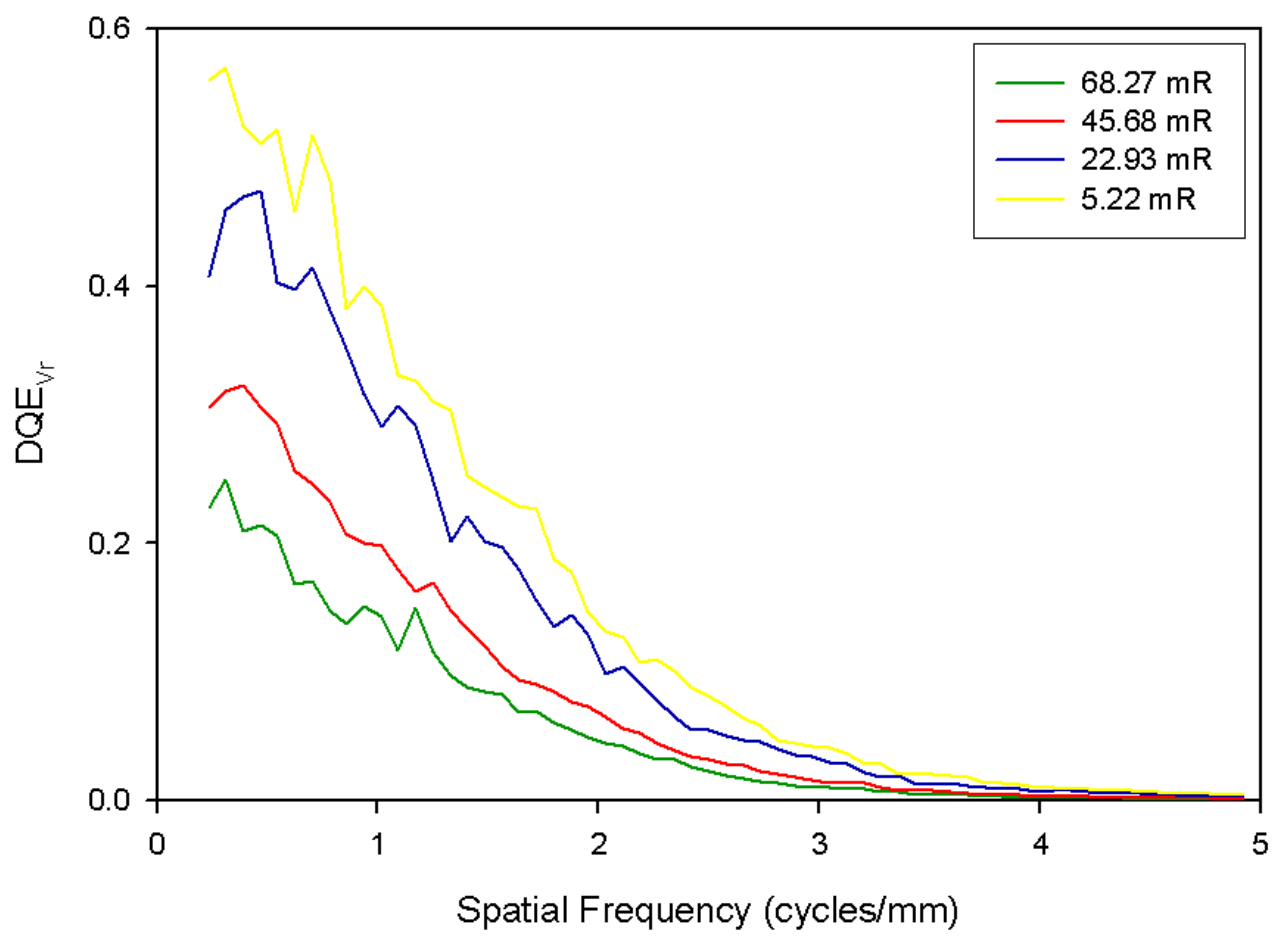

(b)

Figure 7.9: The DQE in (a) horizontal and (b) vertical directions.

Not shown are error bars showing $7 \% \sim 9 \%$ variation at the frequencies displayed. 


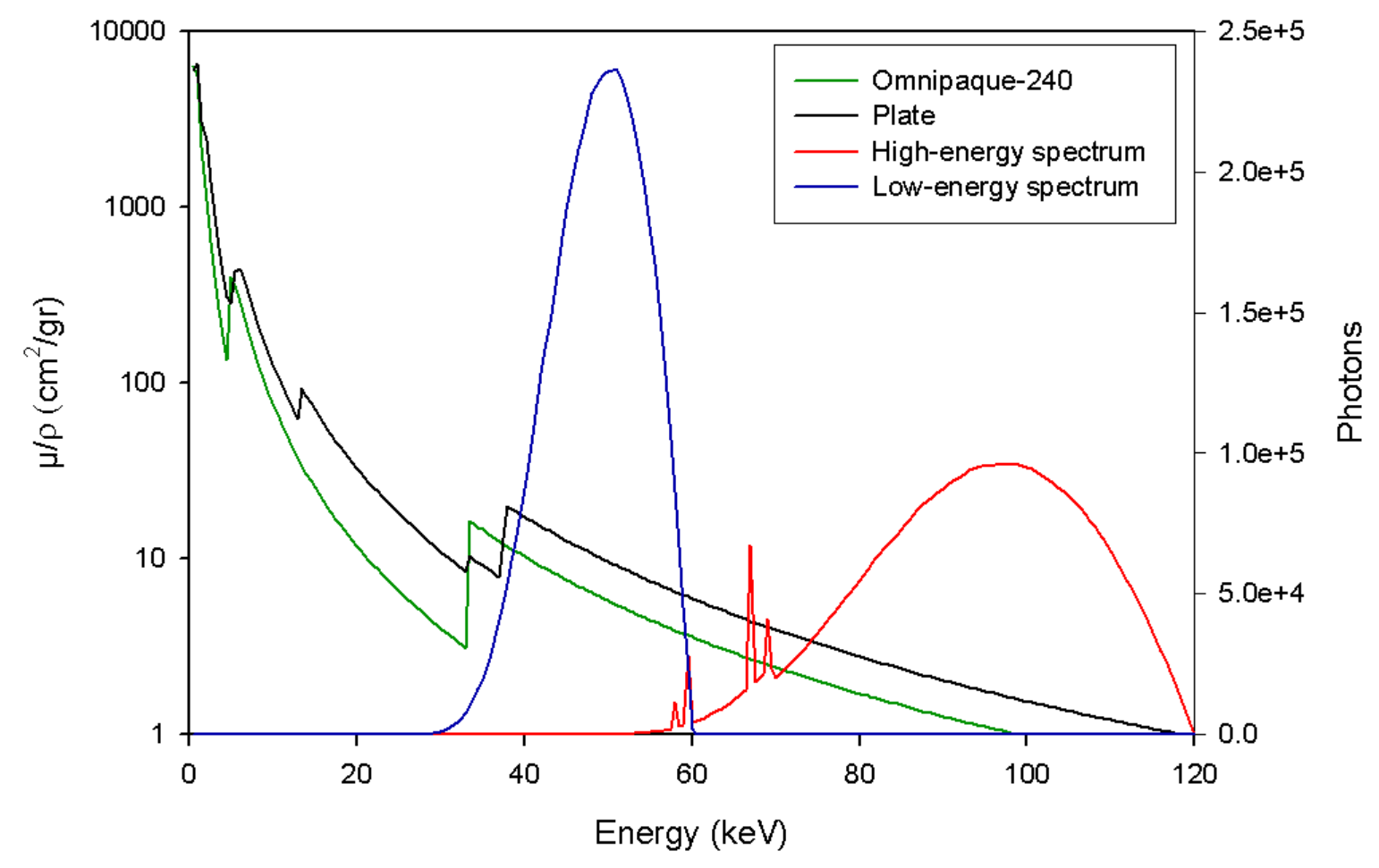

Figure 7.10: High- and low-energy spectra, $\mathrm{a}=1 \mathrm{~mm}^{2}$, (right y-axis) along with plots of logarithmic mass attenuation coefficients of Omnipaque-240 and CR plate (left y-axis).

The spectra are filtered such that both stay above the K-edges of Omnipaque-240 and plate. 
tube heat loading. The high- and low-energy spectra were produced using the same tungsten-anode tube described in Chapter 4, operated at 120 and $60 \mathrm{kVp}$, respectively. In addition to $2.7 \mathrm{~mm}$ inherent and collimator Al-equivalent filtrations, 18- $\mathrm{mm} \mathrm{Al}$ and 4-mm $\mathrm{Cu}$ filters were added to create a hardened high-energy spectrum with mean energy of $93.5 \mathrm{keV}$. The low-energy spectrum was obtained by adding $11.7-\mathrm{mm} \mathrm{Al}$ and $0.5-\mathrm{mm} \mathrm{Cu}$ external filters, producing a hardened low-energy spectrum with mean energy of $48.6 \mathrm{keV}$. The mAs was set to 300 and 600 at 120 and $60 \mathrm{kVp}$, respectively, ensuring sufficient flux being delivered, while keeping the tube heat loading at each exposure within a safe range from the nominal $40 \mathrm{~kW}$.

Also shown in Figure 7.10 are the logarithmic mass attenuation coefficients of Omnipaque-240 and CR plate. Note the positions of spectra with respect to the K-edge of iodine $(33.17 \mathrm{keV})$ in Omnipaque-240 and K-edge of barium $(37.44 \mathrm{keV})$ in the plate.

\subsubsection{Calibration Procedure}

Figure 7.11 shows the photograph of calibration soft tissue (top) and iodine (bottom) step wedges. The iodine phantom was filled with diluted Omnipaque-240 and 121 dual-energy measurements were sampled as shown in Figure 7.12.

The sampled data were used to calculate multiparameter polynomial coefficients by least squares regression analysis. TableCurve ${ }^{\mathrm{TM}}$ 3D (Jandel Scientific Software, Systat Software Inc., San Jose, CA) was implemented to automate surface fitting process of multiple polynomial and non-polynomial equations and obtain the best fit equations for iodine- $(\mathrm{cm})$ and plexiglas-equivalent thicknesses $(\mathrm{cm})$ as,

$$
\begin{aligned}
& t_{i o}=\frac{a_{i o}+b_{i o} x+c_{i o} y+d_{i o} y^{2}}{1+e_{i o} x+f_{i o} y} \\
& t_{p l}=\frac{a_{p l}+b_{p l} x+c_{p l} y+d_{p l} y^{2}}{1+e_{p l} x+f_{p l} x^{2}+g_{p l} y},
\end{aligned}
$$

where $x$ and $y$ are the high- and low-energy log transmissions, respectively. $a$ through $g$ are the calculated coefficients (Table 7.3).

The fitted iodine and plexiglas surfaces defined in Equation 7.1 are shown in Figures 7.13 and 7.14, respectively along with their corresponding residual deviations between the surface and actual measurements. 


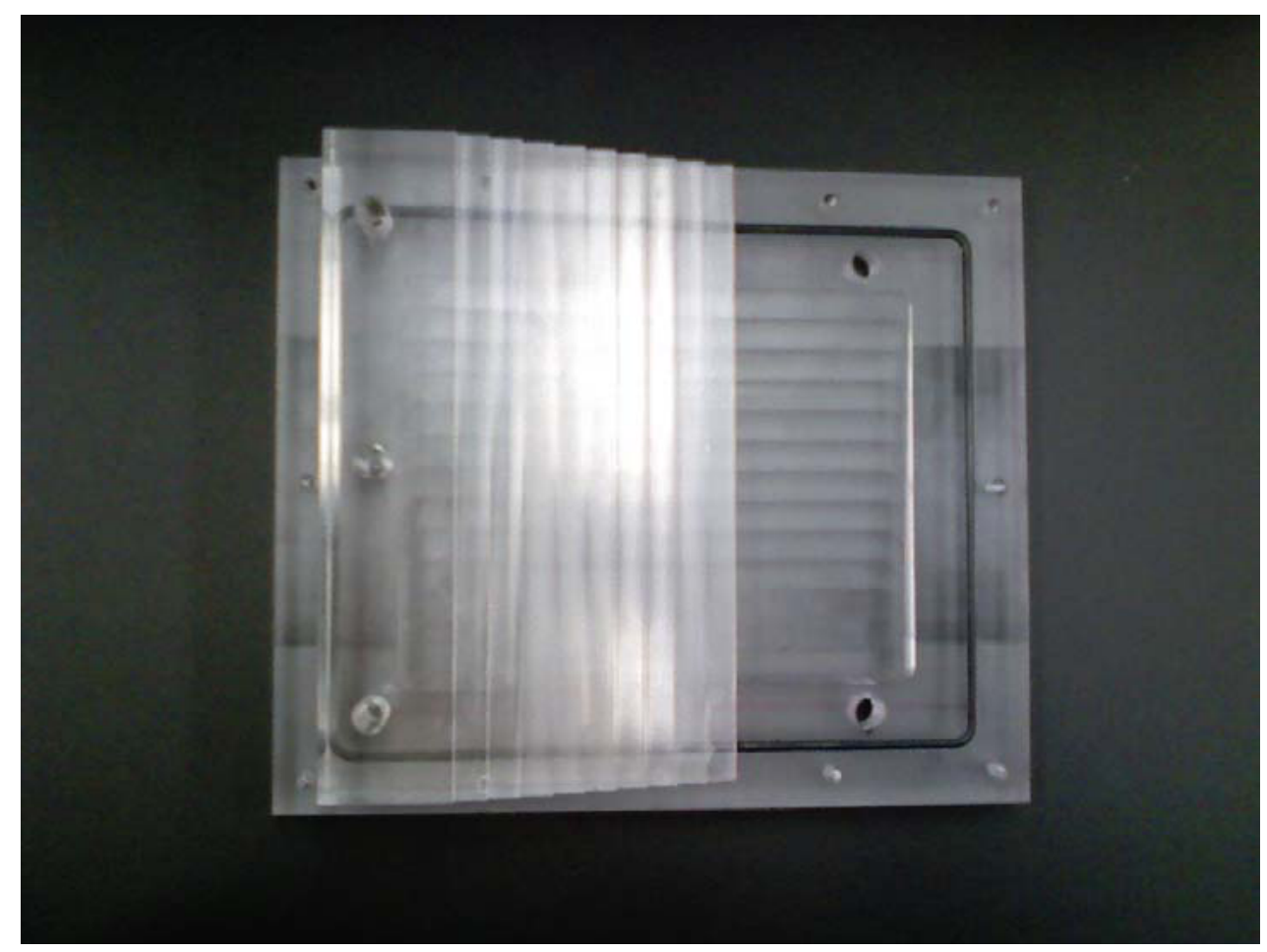

Figure 7.11: Calibration step wedges.

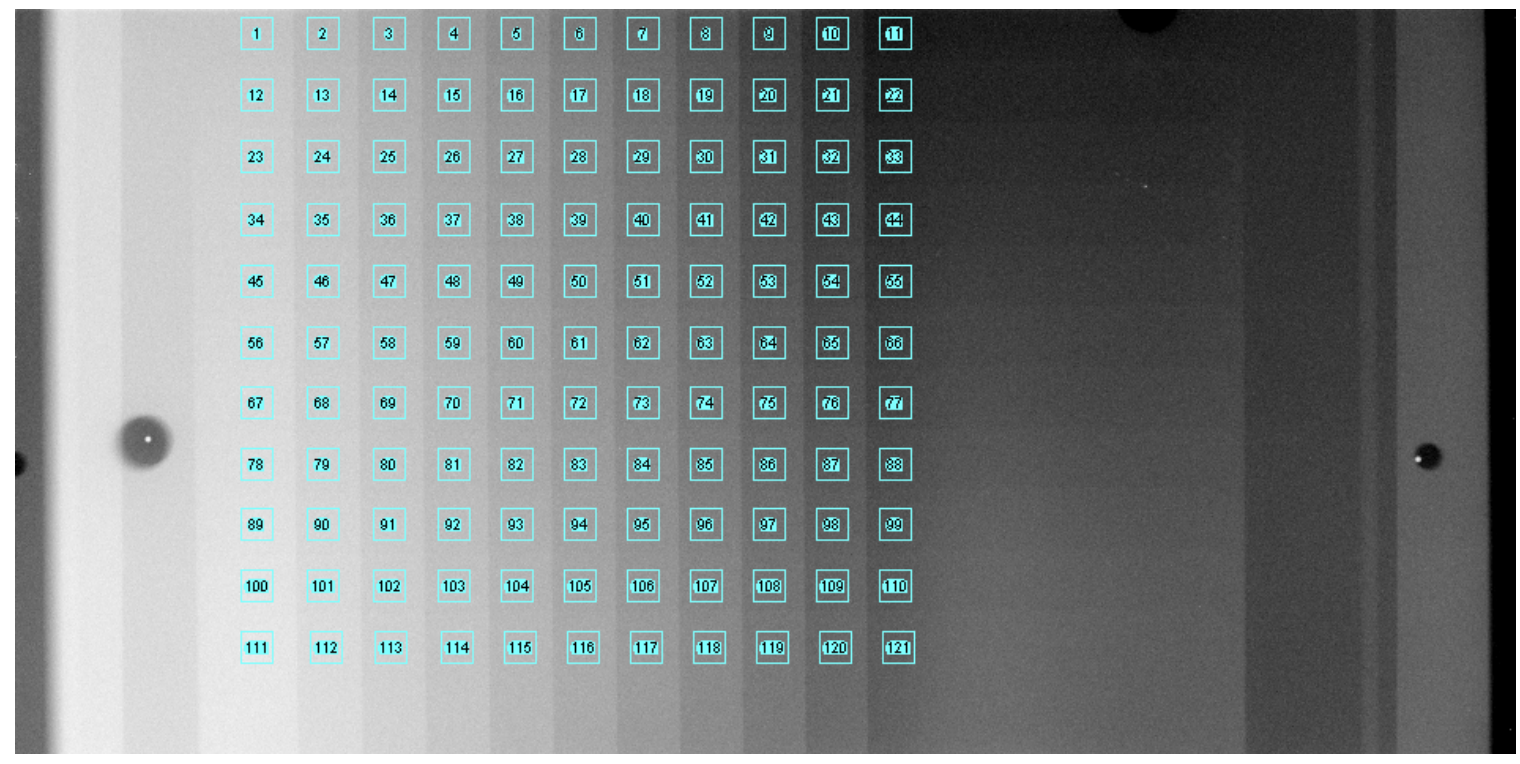

Figure 7.12: Sampled calibration ROIs. 
Table 7.3: Calibration coefficients for the iodine and plexiglas surfaces $\left(R_{\mathrm{io}}^{2}=0.800, R_{\mathrm{pl}}^{2}=0.999\right)$.

\begin{tabular}{ccc}
\hline Parameter & Iodine surface $\left(t_{i o}\right)$ & Plexiglas surface $\left(t_{p l}\right)$ \\
\hline $\mathrm{a}$ & $(8.56 \pm 1.04) \times 10^{-4}$ & 1.46 \\
$\mathrm{~b}$ & $(6.42 \pm 1.78) \times 10^{-2}$ & $8.37 \pm 2.06$ \\
$\mathrm{c}$ & $(-1.85 \pm 0.59) \times 10^{-2}$ & $3.56 \pm 1.69$ \\
$\mathrm{~d}$ & $(-3.16 \pm 0.82) \times 10^{-2}$ & $(1.96 \pm 0.55) \times 10$ \\
$\mathrm{e}$ & $(-2.37 \pm 0.96) \times 10$ & $-8.45 \pm 1.33$ \\
$\mathrm{f}$ & $(1.55 \pm 0.58) \times 10$ & $-4.05 \pm 3.70$ \\
$\mathrm{~g}$ & - & $6.36 \pm 1.76$ \\
\hline
\end{tabular}

\subsubsection{Test Phantom}

Figure 7.15 illustrates the sketch of multi-well plate used as a part of the test phantom. Slabs of catfish fillets were put on top of the plate and exposed to the same high- and low-energy spectra in the calibration procedure to produce high- and low-energy test images shown in Figure 7.16.

The decomposition algorithm described in Equation 7.1 was applied to the highand low-energy test images to produce subtracted soft tissue (soft-tissue only) and iodine (iodine only) images as shown in Figure 7.17.

Note that all radiographic images are displayed ninety-degree clock-wise rotated with respect to the sketch in Figure 7.15. Thus, the first column from the left shows the hemisphere-bottom wells (row \#8 in the sketch).

\subsubsection{Simulated SNR and Dose}

SNR in the subtracted iodine image was measured and theoretically calculated for $1 \mathrm{~mm}^{2}$ of a $3 \mathrm{~cm}$-thick plexiglas containing $1,2,3,5$, and $10 \mathrm{mg} / \mathrm{cm}^{2}$ of Omnipaque240 using the high- and low-energy spectra already described. Figure 7.18 shows measured and modeled SNR, along with measured and modeled SdNR in the temporal subtraction technique, which will be discussed in Section 7.3.

Finally, the theoretical total absorbed dose for one pair of high- and low-energy exposure was estimated to be $2.6 \mathrm{mGy}$ which is significantly less than $\mathrm{LD}_{50 / 30}$ in mice $\left(\mathrm{LD}_{50 / 30}=5-7.6\right.$ Gy depending on the strain of mouse, age at exposure and other factors). 


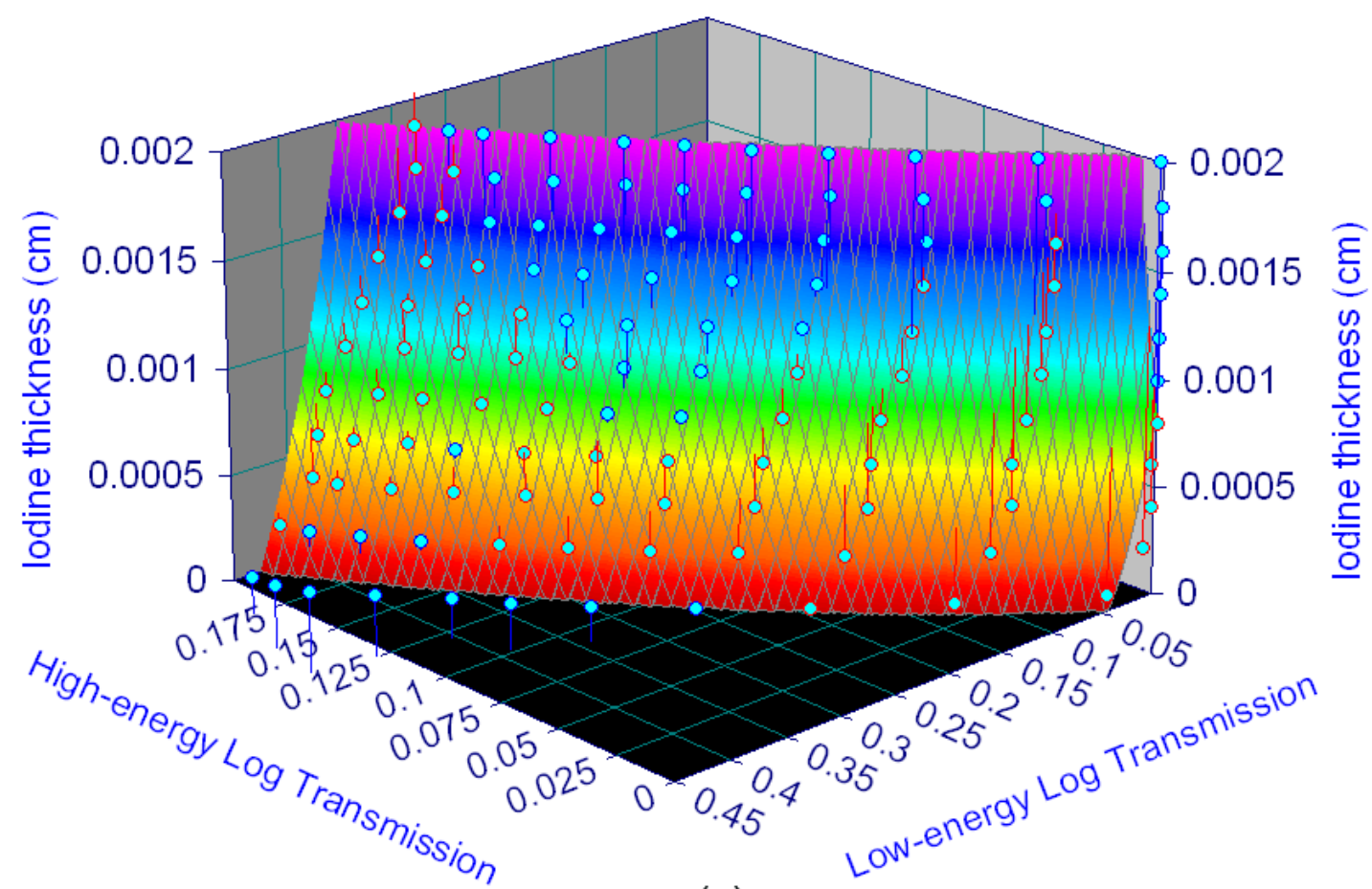

(a)

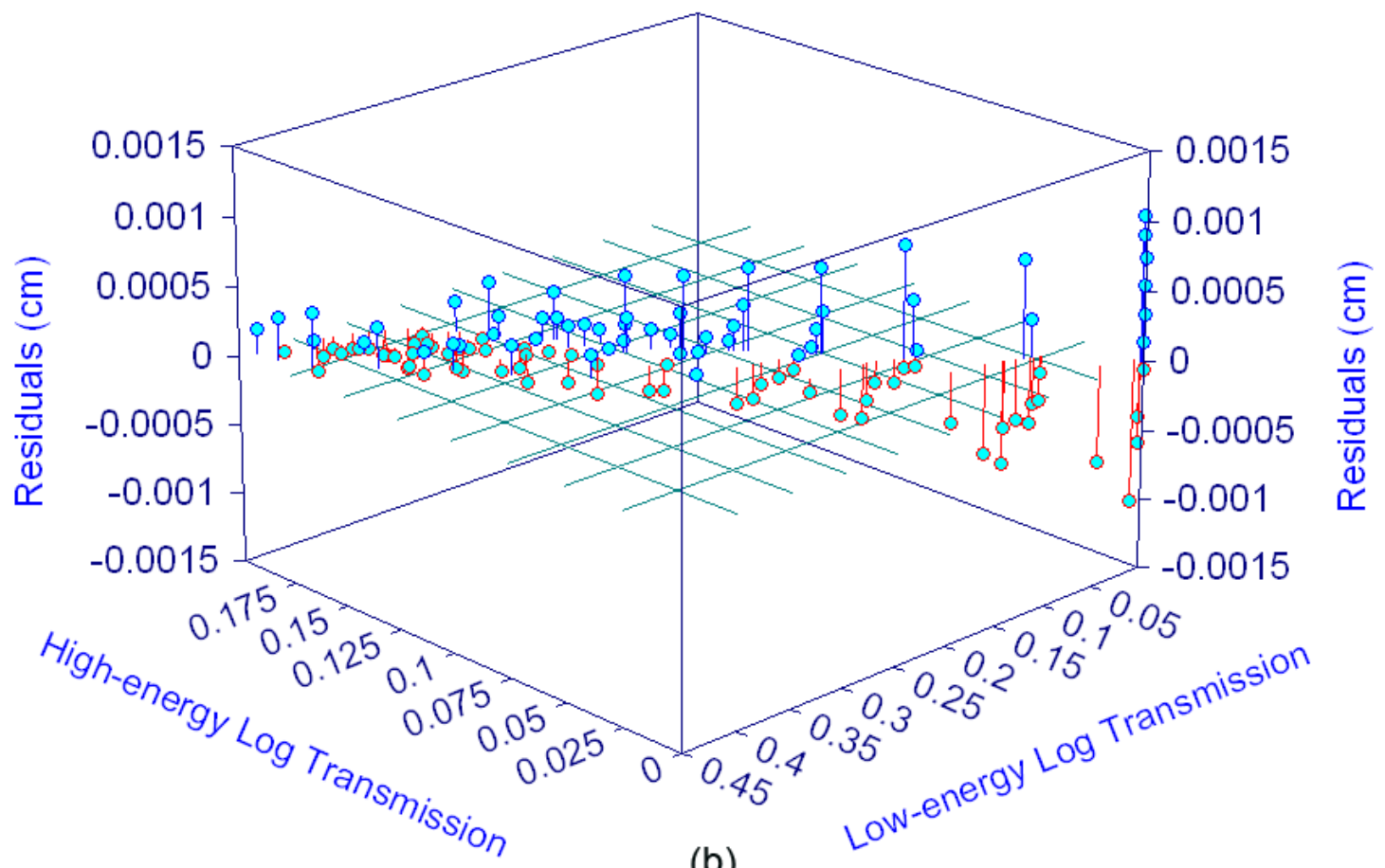

(b)

Figure 7.13: Iodine calibration data.

(a) The surface of iodine thickness plotted against the logarithmic transmissions of the high- and low-energy beams for 121 calibration points. (b) The residual deviations between iodine surface and measurements. The surface fits the measured data to better than $0.005 \mathrm{~mm}$ of iodine for $88 \%$ of calibration points. 


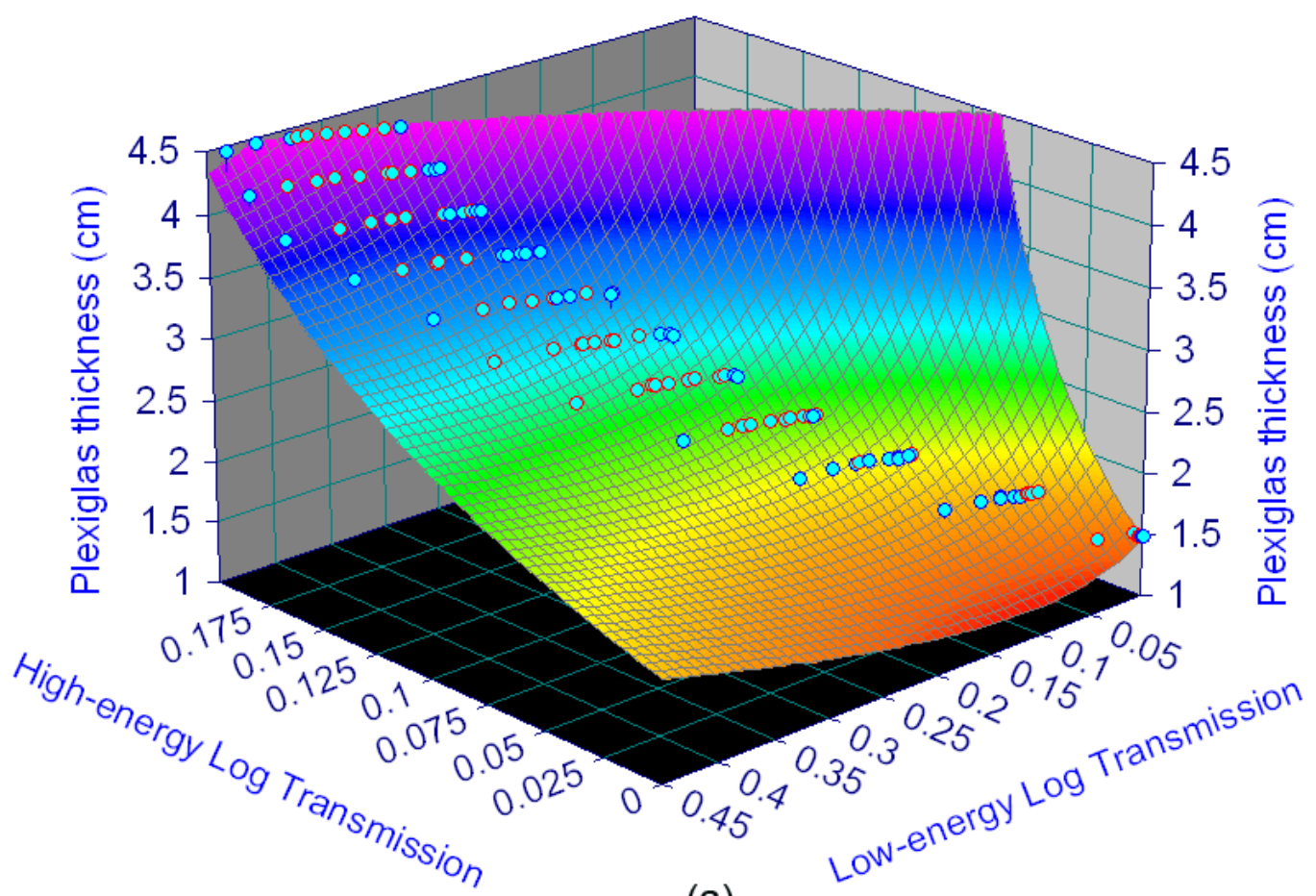

(a)

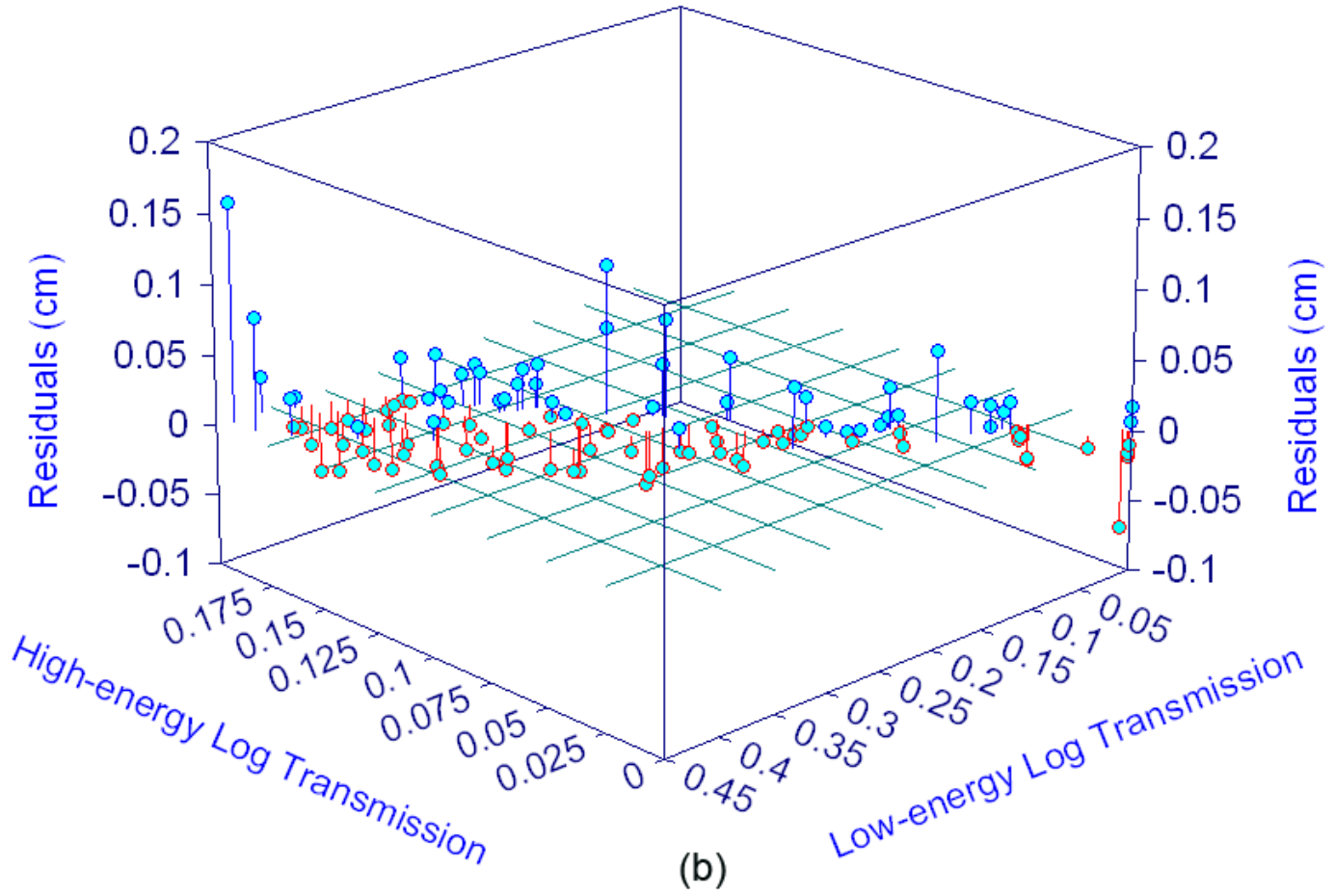

Figure 7.14: Plexiglas calibration data.

(a) The surface of plexiglas thickness plotted against the logarithmic transmissions of the high- and low-energy beams for 121 calibration points. (b) The residual deviations between plexiglas surface and measurements. The surface fits the measured data to better than $0.5 \mathrm{~mm}$ of plexiglas for $94 \%$ of calibration points. 


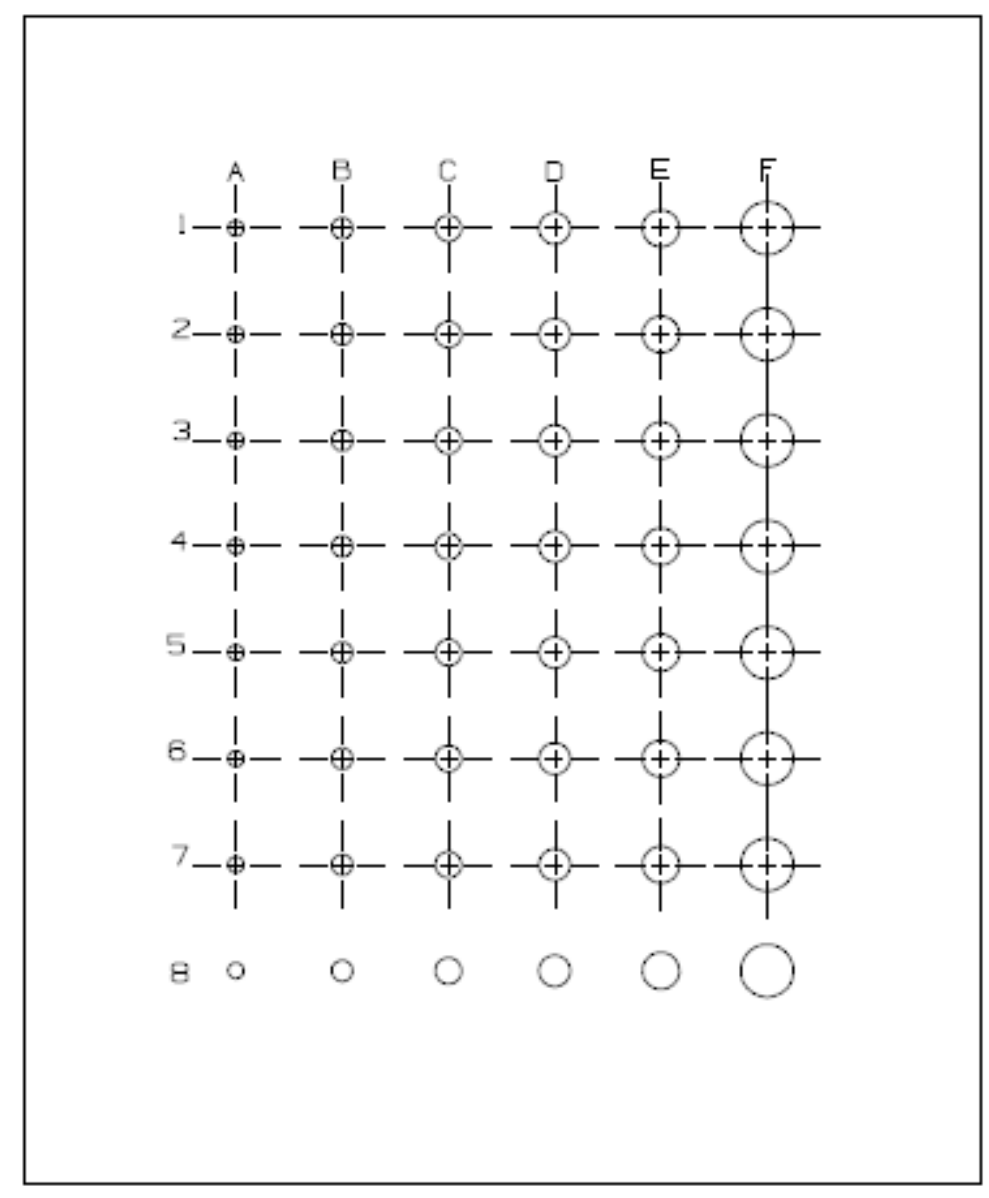

Figure 7.15: The multi-well plexiglas plate as a part of the test phantom. 


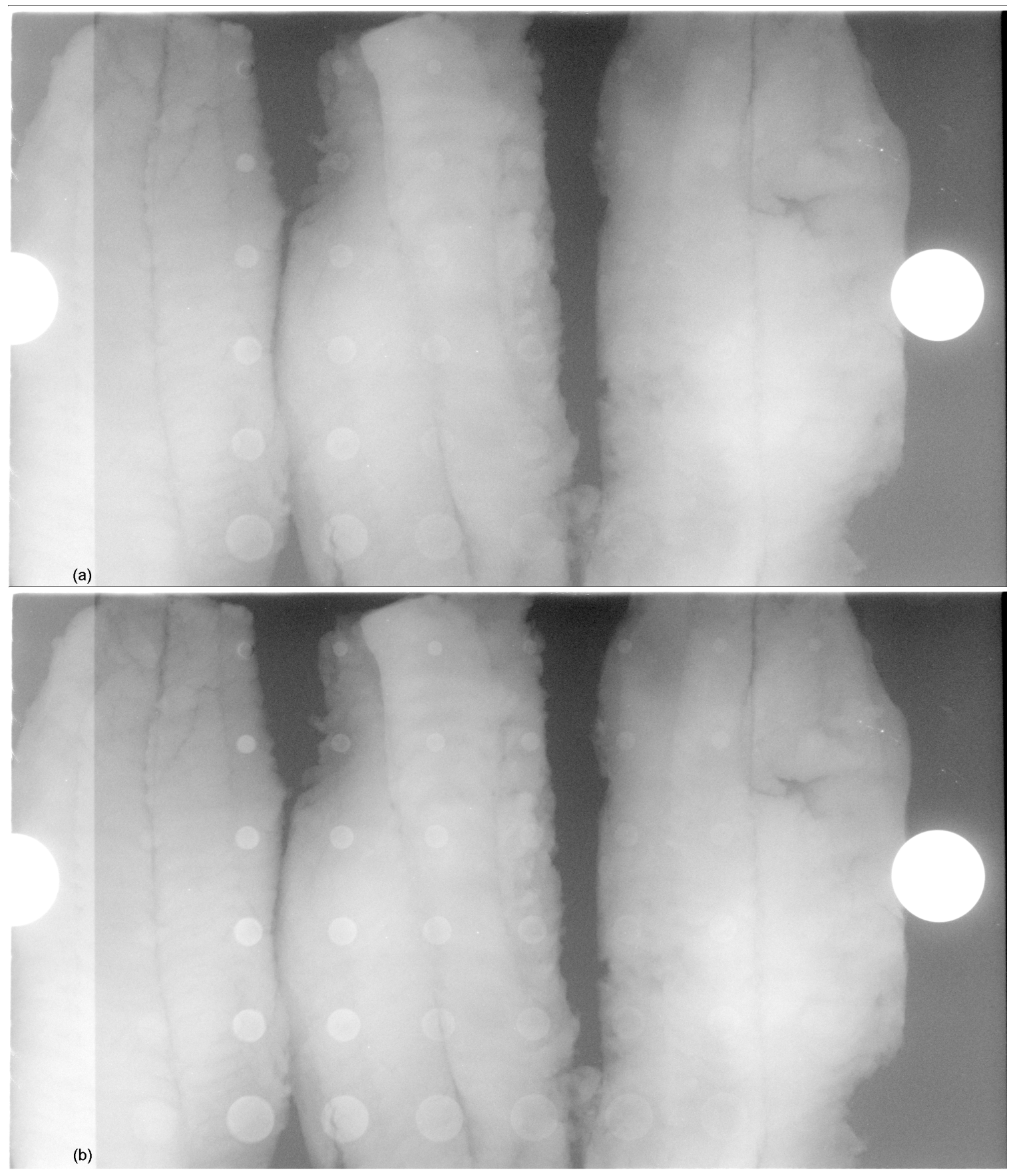

Figure 7.16: (a) High- and (b) low-energy test images. 


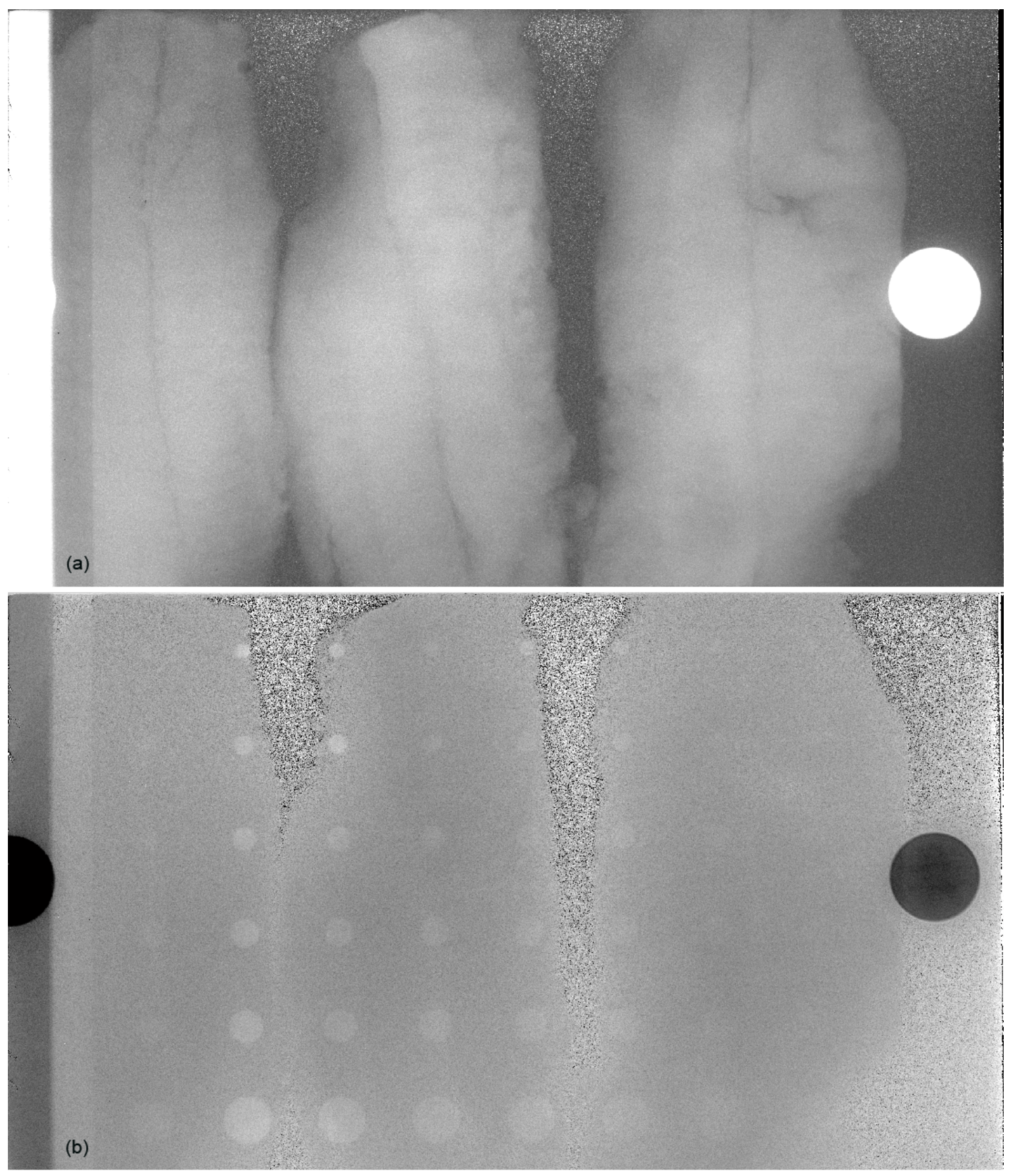

Figure 7.17: (a) Soft tissue-only and (b) iodine-only images. 


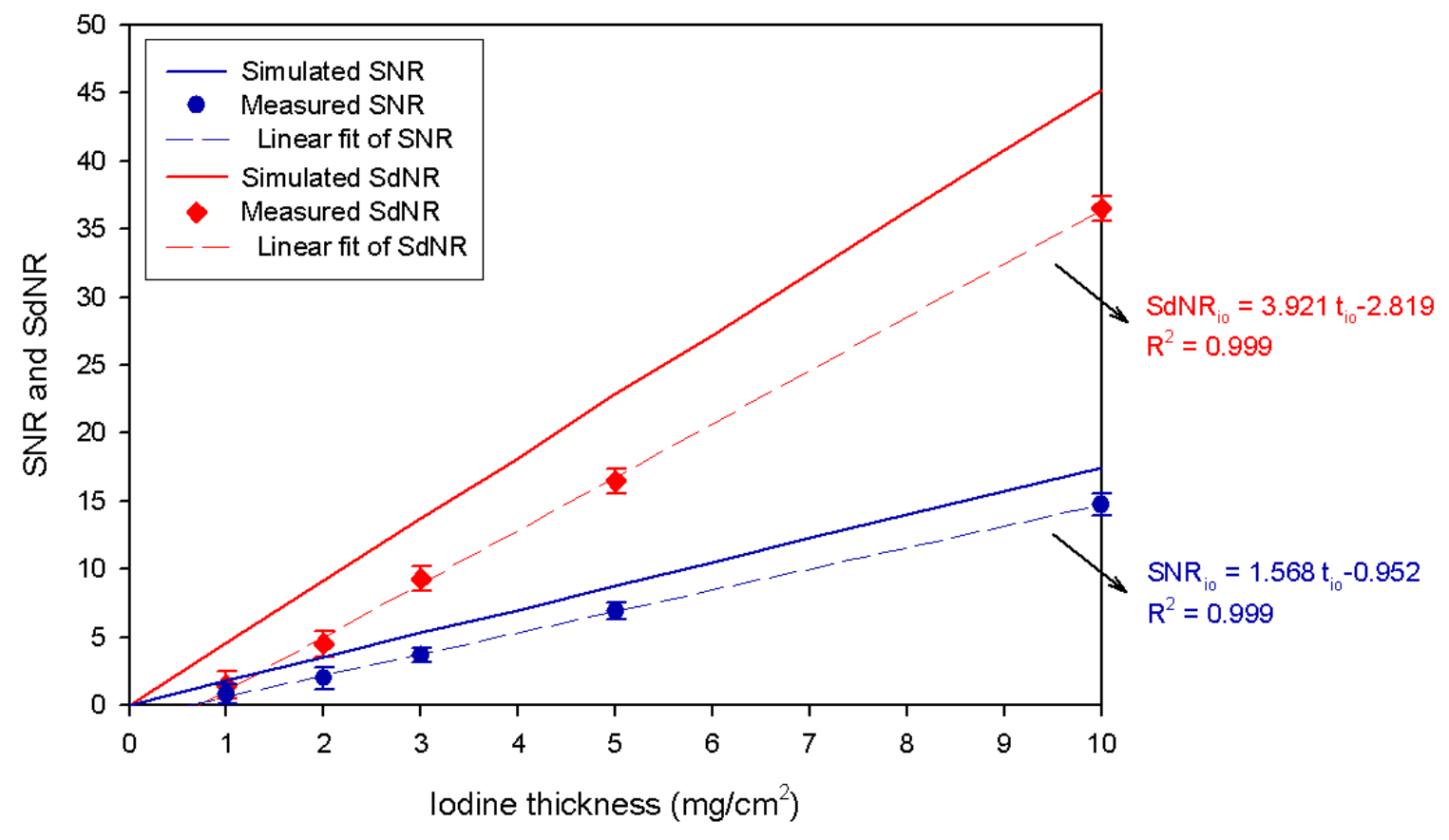

Figure 7.18: Measured and theoretical SNR and SdNR in the dual-energy and temporal subtracted iodine images, respectively. 


\subsection{Temporal Subtraction Technique}

\subsubsection{Spectrum}

The same low-energy spectrum in dual-energy subtraction technique was used to acquire mask and post-contrast images. As a reminder, the low-energy spectrum was obtained by operating the tungsten-anode tube at $60 \mathrm{kVp}, 600 \mathrm{mAs}$, and adding 11.7-mm Al and $0.5-\mathrm{mm} \mathrm{Cu}$ external filters to the tube and collimator combined 2.7-mm inherent Al-equivalent filtration, creating a spectrum with mean energy of $48.6 \mathrm{keV}$.

\subsubsection{Test Phantom}

Figure 7.19 illustrates the mask and post-contrast test images. Three mask and post-contrast images were averaged to obtain the final mask and post-contrast test images prior to subtraction. The subtracted iodine image is displayed in 7.20.

Note that all radiographic images are displayed ninety-degree clock-wise rotated with respect to the sketch in Figure 7.15. Thus, the first column from the left shows the hemisphere-bottom wells (row $\# 8$ in the sketch).

\subsubsection{Simulated SdNR and Dose}

SdNR in the subtracted image was measured and theoretically calculated for

$1 \mathrm{~mm}^{2}$ of a $3 \mathrm{~cm}$-thick plexiglas containing $1,2,3,5$, and $10 \mathrm{mg} / \mathrm{cm}^{2}$ of Omnipaque-240 using the low-energy spectrum already described (Figure 7.18).

Similar to the dual-energy subtraction technique, the theoretical total absorbed dose for one pair of mask and post-contrast exposure was estimated to be $1.1 \mathrm{mGy}$ which is significantly less than $\mathrm{LD}_{50 / 30}$ in mice.

\subsection{Discussion of the Results}

Considering the suggested projection geometry by the IEC (Figure 4.2), comparing the $\mathrm{CR}$ characterization results to the results of previous studies using a Kodak CR 400/HR at the same scan resolution of $100 \mu \mathrm{m}$ can be misleading due to differences in the scanners, use/not use of cassette, use of the same plate, and measurement methods including beam quality and exposure measurement $[61,59]$. In particular, DQE results are strongly dependent on the measurement of exposure, which can vary due to the response of the exposure meter and/or the measurement geometry [61]. Nevertheless, the measured presampled MTF using the edge methodology, NPS, and 


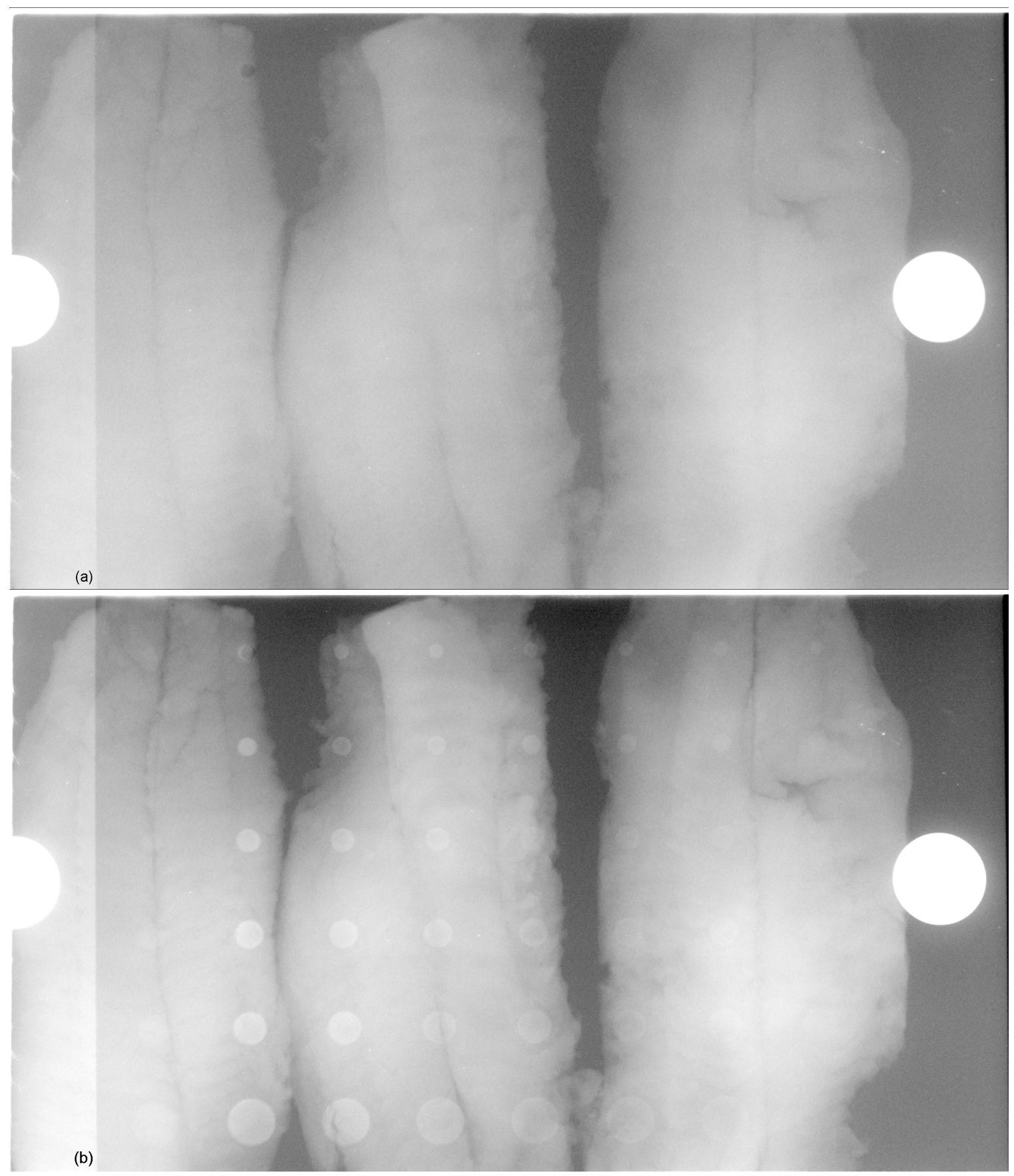

Figure 7.19: (a) Mask and (b) post-contrast images. 


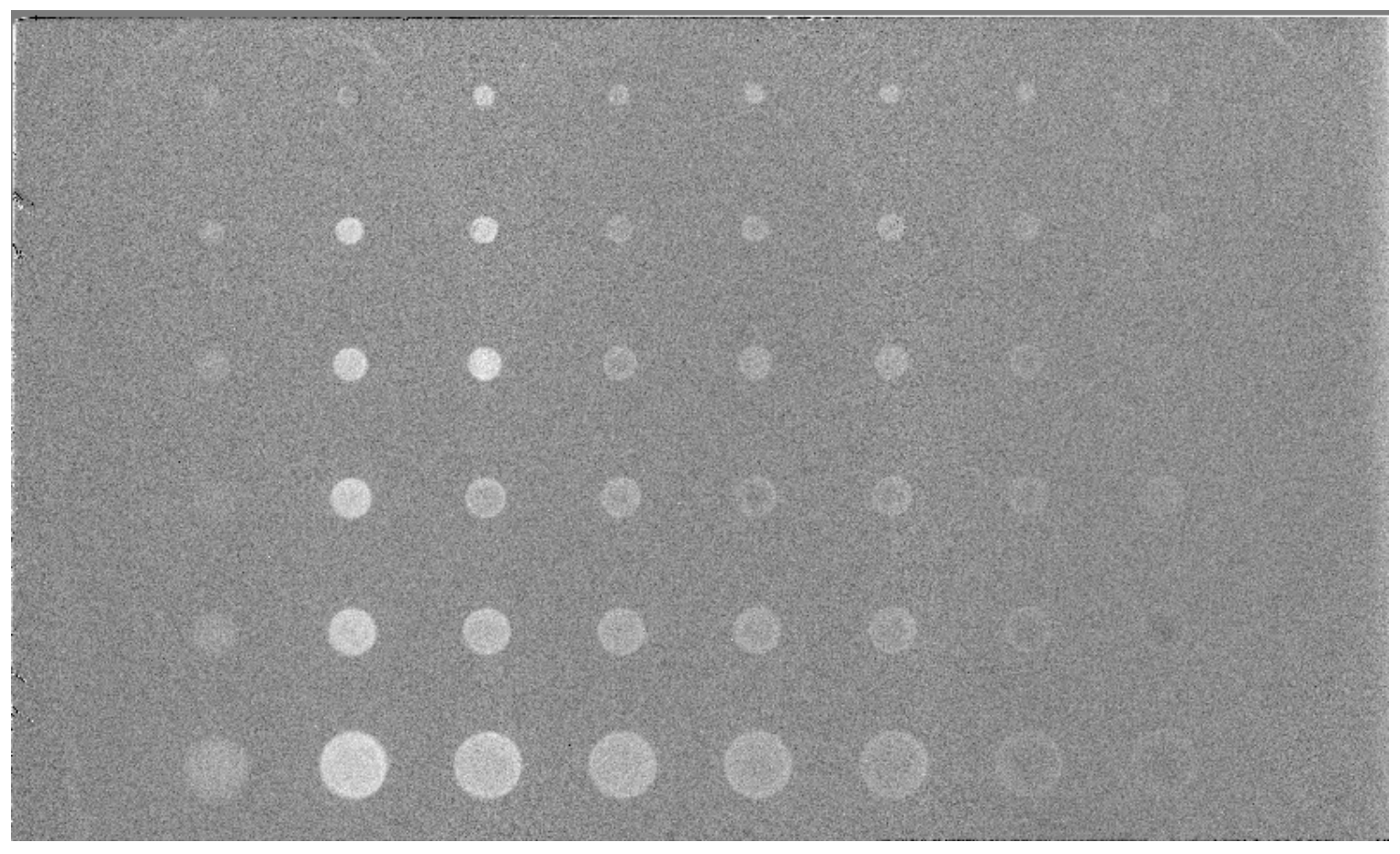

Figure 7.20: Temporal subtracted iodine image. 
DQE of the studied Kodak CR 7400 were fairly close to the results obtained by the Kodak CR 400/HR considering similar exposures.

In both dual-energy and temporal subtraction techniques, the measured SNR and SdNR were always lower than their simulated counterparts. In general, the lower measured SNR and SdNR could be explained by physical phenomena present in the actual experimental set-up but not included in the simulation, such as differences between the true and modeled geometry; deviations between the true and modeled plate, test phantom, and contrast agent; X-ray scatter from the test phantom, plate, plate holder, table, and the surrounding objects; plate non-uniformities; scanner mechanical and electronic imperfections; and focal-spot motion (target wobble, etc.).

The decomposition algorithm in the dual-energy subtraction technique completely removed the iodinated regions in the tissue-only image. However, the iodineonly image ended up with soft tissue residuals. There are different parameters contributing to such residuals. These could be listed as: (1) the accuracy of the calibration procedure, (2) overall sensitivity of the plate and scanner, (3) non-uniformity of the plate not being completely removed by the flat-field correction, (4) low concentration of iodine (small projection thickness) compared to the total thickness of the soft tissue which makes iodine removal more challenging, and (5) using plexiglas in the calibration procedure which does not exactly represent the catfish fillet with $6 \%$ fat in terms of X-ray absorption properties.

In the iodine-only image, the first column from the right containing the 1-mm deep flat-bottom wells has little or no visibility. This is also evident in the Figure 7.18 where the measured SNR for $1 \mathrm{mg} / \mathrm{cm}^{2}$ of iodine is believed to be mainly dominated by the background noise.

The theoretical and measured SdNR at each iodine thickness in the temporal subtraction technique were higher than their corresponding SNR in the dual-energy subtraction technique. In particular, the column for 1-mm deep flat-bottom wells is visible in the temporal subtracted iodine image. This was expected since the SNR in dual-energy subtraction technique was obtained by subtracting the high- and lowenergy images of the same iodinated regions with close contrasts, while SdNR in temporal subtraction technique is the result of subtracting the low-contrast mask from the high-contrast post-contrast image.

Functional imaging in small animals can be addressed using both dual-energy and temporal subtraction techniques. However, acquiring images synchronous to biological rhythms, such as cardiac or ventilatory cycles present challenges.

One of the key aspects and advantages of implementing dual-energy subtraction technique in clinical or live small animal studies is very rapid switching between the acquisitions of the high- and low-energy images, which makes this technique less sensitive to motion of the object being imaged. This has necessitated designing X-ray tubes in which switching between high and low $\mathrm{kVps}$ and their corresponding filters is done within a few milliseconds. These X-ray tubes were originally designed for 
human clinical exams in the 1970s and later incorporated in the hybrid DSA systems in the 1980s $[19,17]$.

Assuming using such an X-ray tube in the current experiment to image a live small animal with gated cardiac and ventilatory cycles, it is required to capture both images on the same CR plate at once and read together. Therefore, there is a need for a mechanical plate holder working in parallel with the X-ray tube switching to move plate into the field of view such that high-energy image is captured on one part of the plate and low-energy image on another part. Then the high- and low-energy images are to be registered before applying the decomposition algorithm.

The temporal subtraction approach offers the possibility to analyze the enhancement and washout patters of the contrast agent into and out of the lesion. However, in order to get artifact-free subtracted images, the mask and post-contrast images must be registered with a high accuracy. This substantiates the need for cardiac and ventilatory gating. 


\section{Chapter 8}

\section{Conclusions}

A comprehensive study of the application of a Kodak high-resolution compact computed radiography system (CR 7400) in detecting an iodinated contrast agent in a small animal model is presented in this dissertation. First, the imaging performance of the CR was characterized by measuring presampled MTF, NPS, RSD, NEQ and DQE. Then, dual-energy and temporal subtraction techniques were applied on a small animal phantom in order to enhance the contrast of the iodinated regions and suppress soft tissue background. In the end, both techniques were compared by evaluating how effective each one is in removing the soft tissue background, including calculating and measuring SNR in the subtracted iodine image.

The discrepancies between the experimental and theoretical SNR curves in dualenergy and temporal subtraction techniques could be explained by the physical phenomena present in the actual experimental set-up but not included in the simulation (Section 7.4). Temporal subtraction technique produced a subtracted iodine image with higher SNR and almost no soft tissue background residuals. There are different parameters contributing to the residuals found in the dual-energy subtracted iodine image, which were all discussed in Section 7.4.

The CR 7400 plates were used without any cassettes in this project. They can also be loaded into the cassettes manually. In both cases, handling the plates directly subjects them to various physical damages such as cracks and wear-and-tear. In order to minimize such damages, it is preferable to have a plate reading mechanism in which the cassette containing the plate is inserted into the reader, then the plate is fetched, read, and placed back into the cassette. Moreover, the CR 7400 plates are to be seated on the scanner drum prior to reading, which causes the plates to lose rigidity after a prolonged use. Consequently, the flat-bed readers are preferred.

Recent advances in the design of CR PSP plates have made it possible to manufacture plates made of BaFI:Eu [51]. This type of plate has proved to have lower structure noise and DQE superior to the traditional $\mathrm{BaF}(\mathrm{Br}, \mathrm{I})$ :Eu plates. Such plates will be an ideal fit for this study in which detection of very low concentrations of iodine is important.

In conclusion, the current results suggest the use of temporal subtraction technique in small animal studies, particularly to detect zone of angiogenesis, as a means

for tracking tumor growth. It can also provide information about the kinetics of the contrast agent. However, as discussed in Section 7.4, cardiac and ventilatory gating 
are necessary to produce artifact-free subtracted images. Overall, the study supports the potential of low-cost CR 7400 in small animal studies, particularly detecting iodinated contrast agents implementing temporal subtraction technique. The topics and results discussed in this dissertation provide a background to study the application of $\mathrm{CR}$ in imaging live small animals. 


\section{List of References}

[1] IEC 62220-1 Ed. 1: Medical electrical equipment - characteristics of digital X-ray imaging devices - Part 1: Determination of the detective quantum efficiency, 2003.

[2] H. Aichinger, J. Dierker, S. Joite-Barfuß, and M. Sabel, Radiation Exposure and Image Quality in X-ray Diagnostic Radiology: Physical Principles and Clinical Applications. Berlin, Germany: Springer-Verlag, 2004.

[3] R. E. Alvarez and A. Macovski, "Energy-selective reconstructions in X-ray computerized tomography," Physics in Medicine and Biology, vol. 21, no. 5, pp. 733-744, 1976.

[4] R. E. Alvarez, "Active energy selective image detector for dual-energy computed radiography," Medical Physics, vol. 23, no. 10, pp. 1739-1748, 1996.

[5] R. E. Alvarez, J. A. Seibert, and S. K. Thompson, "Comparison of dual-energy detector system performance," Medical Physics, vol. 31, no. 3, pp. 556-565, 2004.

[6] K. Aoki, "Computed radiography in pediatrics," Journal of Digital Imaging, vol. 8, pp. 92-96, 1995.

[7] C. D. Arvanitis, G. Royle, and R. Speller, "Dual-energy contrast enhancement breast imaging opimization using contrast to noise ratio," Proceedings of SPIE, vol. 6510, pp. 65 102Y:1-65 102Y:10, 2007.

[8] C. Badea, L. W. Hedlund, and G. A. Johnson, "Micro-CT with respiratory and cardiac gating," Medical Physics, vol. 31, no. 12, pp. 3324-3329, 2004.

[9] C. Badea, M. Drangova, D. Holdsworth, and G. Johnson, "In vivo small animal imaging using micro-CT and digital subtraction angiography," Physics in Medicine and Biology, vol. 53, pp. R319-R350, 2008.

[10] H. H. Barrett and W. Swindell, Radiological Imaging: The Theory of Image Formation, Detection, and Processing. Waltham, MA: Academic Press, 1981.

[11] J. M. Boone, O. Velasquez, and S. R. Cherry, "Small-animal X-ray dose from micro-CT," Molecular Imaging, vol. 3, no. 3, pp. 149-158, 2004.

[12] M. Boone, Handbook of Medical Imaging: Physics and Psychophysics. Bellingham, WA: Society of Photo-Optical Instrumentation Engineers (SPIE), 2000, vol. 1, ch. X-ray production, interaction, and detection in diagnostic imaging. 
[13] G. Borasi, A. Nitrosi, P. Ferrari, and D. Tassoni, "On site evaluation of three flat panel detectors for digital radiography," Medical Physics, vol. 30, no. 7, pp. 1719-1731, 2003.

[14] C. D. Bradford, W. W. Peppler, and J. T. D. III, "Performance characteristics of a Kodak computed radiography system," Medical Physics, vol. 26, no. 1, pp. 27-37, 1999.

[15] W. R. Brody, Digital Radiography. New York, NY: Raven Press, 1984.

[16] W. R. Brody, A. Macovski, N. J. Pelc, L. Lehmann, R. A. Joseph, and L. Edelheit, "Intravenous arteriography using scanned projection radiography," Radiology, vol. 141, no. 2, pp. 509-514, 1981.

[17] W. R. Brody, "Hybrid subtraction for improved arteriography," Radiology, vol. 141, no. 3, pp. 828-831, 1981.

[18] W. R. Brody, G. Butt, A. Hall, and A. Macovski, "A method for selective tissue and bone visualization using dual energy scanned projection radiography," Medical Physics, vol. 8, no. 3, pp. 353-357, 1981.

[19] W. R. Brody, D. M. Cassel, F. G. Sommer, L. A. Lehmann, A. Macovski, R. E. Alvarez, N. J. Pelc, S. J. Riederer, and A. L. Hall, "Dual-energy projection radiography: initial clinical experience," American Journal of Radiology, vol. 137, pp. 201-205, 1981.

[20] R. L. Burden and J. D. Faires, Numerical Analysis, 9th ed. Stamford, CT: Brooks Cole, 2010.

[21] J. T. Bushberg, J. A. Seibert, E. M. L. Jr., and J. M. Boone, The Essential Physics of Medical Imaging, 2nd ed. Philadelphia, PA: Lippincott Williams \& Wilkins, 2001.

[22] C. H. J. Chang, D. E. Nesbit, D. R. Fisher, S. L. Fritz, S. J. Dwyer III, A. W. Templeton, F. Lin, and W. R. Jewell, "Computed tomographic mammography using a conventional body scanner," American Journal of Roentgenology, vol. 138, no. 3, pp. 553-558, 1982.

[23] C. H. J. Chang, J. L. Sibala, S. L. Fritz, J. H. Gallagher, S. J. Dwyer III, and A. W. Templeton, "Computed tomographic evaluation of the breast," American Journal of Roentgenology, vol. 131, no. 3, pp. 459-464, 1978.

[24] A. R. Cowen, A. Workman, and J. S. Price, "Physical aspects of photostimulable phosphor computed radiography," The British Journal of Radiology, vol. 66, no. 784, pp. 332-345, 1993.

[25] P. P. Dendy and B. Heaton, Physics for Diagnostic Radiology, 2nd ed. London, UK: Institute of Physics Publishing, 1999. 
[26] J. T. Dobbins III, D. L. Ergun, L. Rutz, D. A. Hinshaw, H. Blume, and D. C. Clark, "DQE(f) of four generations of computed radiography acquisition devices," Medical Physics, vol. 22, no. 10, pp. 1581-1593, 1995.

[27] M. J. Flynn and E. Samei, "Experimental comparison of noise and resolution for $2 \mathrm{k}$ and $4 \mathrm{k}$ storage phosphor radiography systems," Medical Physics, vol. 26, no. 8, pp. 1612-1623, 1999.

[28] J. Folkman and M. Klagsbrun, "Angiogenic factors," Science, vol. 235, no. 4787, pp. $442-447,1987$.

[29] J. Folkman, K. Watson, D. Ingber, and D. Hanahan, "Induction of angiogenesis during the transition from hyperplasia to neoplasia," Nature, vol. 339, pp. 58-61, 1989.

[30] N. L. Ford, M. M. Thornton, and D. W. Holdsworth, "Fundamental image quality limits for microcomputed tomography in small animals," Medical Physics, vol. 30, no. 11, pp. 2869-2877, 2003.

[31] N. L. Ford, K. C. Graham, A. C. Groom, I. C. MacDonald, A. F. Chambers, and D. W. Holdsworth, "Time-course characterization of the computed tomography contrast cnhancement of an iodinated blood-pool contrast agent in mice using a volumetric flat-panel equipped computed tomography scanner," Investigative Radiology, vol. 41, no. 4, pp. 384-390, 2006.

[32] D. M. Gauntt and G. T. Barnes, "X-ray tube potential, filtration, and detector considerations in dual-energy chest radiography," Medical Physics, vol. 21, no. 2, pp. 203-218, 1994.

[33] A. L. Goertzen, V. Nagarkar, R. A. Street, M. J. Paulus, J. M. Boone, and S. R. Cherry, "A comparison of X-ray detectors for mouse CT imaging," Physics in Medicine and Biology, vol. 49, no. 23, pp. 5251-5265, 2004.

[34] D. P. Harrington, L. M. Boxt, and P. D. Murray, "Digital subtraction angiography: overview of technical principles," American Journal of Roentgenology, vol. 139, no. 4, pp. 781-786, 1982.

[35] J. Heinzerling and M. Schlindwein, "Nonlinear techniques in multispectral X-ray imaging," IEEE Transactions on Nuclear Science, vol. 27, no. 2, pp. 961-968, 1980.

[36] W. R. Hendee and E. R. Ritenour, Medical Imaging Physics. Hoboken, NJ: Wiley-Liss, 2002.

[37] D. Hoeschen, "DQE of digital X-ray imaging systems: a challenge for standardization," Proceedings of SPIE, vol. 4320, pp. 280-286, 2001. 
[38] J. Hsieh, Computed Tomography: Principles, Designs, Artifacts, and Recent Advances. Bellingham, WA: Society of Photo-Optical Instrumentation Engineers (SPIE), 2003.

[39] J. H. Hubbell and S. M. Seltzer, "NIST standard reference database 126: Tables of X-Ray mass attenuation coefficients and mass energy-absorption coefficients from $1 \mathrm{keV}$ to $20 \mathrm{MeV}$ for elements $\mathrm{Z}=1$ to 92 and 48 additional substances of dosimetric interest," 2004. [Online]. Available: http://www.nist.gov/pml/data/xraycoef/index.cfm

[40] G. F. K. Cranley, B. Gilmore and L. Desponds, "IPEM report 78: catalog of diagnostic X-ray spectra and other data," The Institute of Physics and Engineering in Medicine (IPEM), Tech. Rep., 1997.

[41] S. M. Kengyelics, J. H. Launders, and A. R. Cowen, "Physical imaging performance of a compact computed radiography acquisition device," Medical Physics, vol. 25, no. 3, pp. 354-360, 1998.

[42] L. A. Lehmann and R. E. Alvarez, Digital Radiography: Selected Topics. Berlin, Germany: Springer-Verlag, 1986, ch. Energy selective radiography: a review.

[43] L. A. Lehmann, R. E. Alvarez, A. Macovski, W. R. Brody, N. J. Pelc, S. J. Riederer, and A. L. Hall, "Generalized image combinations in dual kVp digital radiography," Medical Physics, vol. 8, no. 5, pp. 659-667, 1981.

[44] M. R. Lemacks, S. C. Kappadath, C. C. Shaw, X. Liu, and G. J. Whitman, "A dual-energy subtraction technique for microcalcification imaging in digital mammography: a signal-to-noise analysis," Medical Physics, vol. 29, no. 8, pp. 1739-1751, 2002.

[45] D. C. Levin, R. M. Schapiro, L. M. Boxt, L. Dunham, D. P. Harrington, and D. L. Ergun, "Digital subtraction angiography: principles and pitfalls of image improvement techniques," American Journal of Roentgenology, vol. 143, no. 3, pp. 447-454, 1984.

[46] M. D. Lin, E. Samei, C. T. Badea, T. T. Yoshizumi, and G. A. Johnson, "Optimized radiographic spectra for small animal digital subtraction angiography," Medical Physics, vol. 33, no. 11, pp. 4249-4257, 2006.

[47] F. E. Lindhardt, "Clinical experiences with computed radiography," European Journal of Radiology, vol. 22, pp. 175-185, 1996.

[48] A. Macovski, Medical Imaging Systems. Upper Saddle River, NJ: Prentice-Hall, 1983.

[49] K. A. Miles and M. R. Griffiths, "Perfusion CT: a worthwhile enhancement?" British Institute of Radiology, vol. 76, pp. 220-231, 2003. 
[50] J. P. Moy and B. Bosset, "How does real offset and gain correction affect the DQE in images from X-ray flat detectors?" Proceedings of SPIE, vol. 3659, pp. 90-97, 1999.

[51] Y. Nakano, T. Gido, S. Honda, A. Maezawa, H. Wakamatsu, and T. Yanagita, "Improved computed radiography image quality from a BaFI:Eu photostimulable phosphor plate," Medical Physics, vol. 29, no. 4, pp. 592-597, 2002.

[52] O. Nalcioglu and R. Y. Lou, "New methods for beam hardening correction in computed tomography," Radiology, vol. 125, 1977.

[53] R. R. O. Rampado, P. Isoardi, "Quantitative assessment of computed radiography quality control parameters," Physics in Medicine and Biology, vol. 51, pp. 1-17, 2006.

[54] M. E. Phelps, E. J. Hoffman, and M. M. Ter-Pogosian, "Attenuation coefficients of various body tissues, fluids, and lesions at photon energies of 18 to $136 \mathrm{keV}$," Radiology, vol. 117, pp. 573-583, 1975.

[55] S. Rivetti, N. Lanconelli, M. Bertolini, A. Nitrosi, A. Burani, and D. Acchiappati, "Comparison of different computed radiography systems: physical characterization and contrast detail analysis," Medical Physics, vol. 37, no. 2, pp. 440-448, 2010.

[56] J. A. Rowlands, "The physics of computed radiography," Physics in Medicine and Biology, vol. 47, pp. R123-R166, 2002.

[57] E. Samei, M. J. Flynn, H. G. Chotas, and J. T. Dobbins, "DQE of direct and indirect digital radiographic systems," Proceedings of SPIE, vol. 4320, pp. 189197, 2001.

[58] E. Samei, "Image quality in two phosphor-based flat panel digital radiographic detectors," Medical Physics, vol. 30, no. 7, pp. 1747-1757, 2003.

[59] E. Samei and M. J. Flynn, "An experimental comparison of detector performance for computed radiography systems," Medical Physics, vol. 29, no. 4, pp. 447-459, 2002.

[60] E. Samei, M. J. Flynn, and D. A. Reimann, "A method for measuring the presampled mtf of digital radiographic systems using an edge test device," Medical Physics, vol. 25, no. 1, pp. 102-113, 1998.

[61] E. Samei, J. A. Seibert, C. E. Willis, M. J. Flynn, E. Mah, and K. L. Junck, "Performance evaluation of computed radiography systems," Medical Physics, vol. 28 , no. 3, pp. 361-371, 2001.

[62] R. Schaetzing, "Computed radiography technology," in Advances in digital radiography: categorical course in diagnostic radiology physics, E. Samei and M. Flynn, Eds. Radiological Society of North America (RSNA), 2003. 
[63] J. A. Seibert, "Computed radiography technology," Medical Physics Publishing, 2004.

[64] J. A. Seibert, T. M. Bogucki, T. Ciona, W. Huda, A. Karellas, J. R. Mercier, E. Samei, S. J. Shepard, B. K. Stewart, K. J. Strauss, O. H. Suleiman, D. Tucker, R. A. Uzenoff, J. C. Weiser, and C. E. Willis, "AAPM report no. 93: acceptance testing and quality control of photostimulable storage phosphor imaging systems," American Association of Physicists in Medicine (AAPM), Tech. Rep., 2006, report of AAPM Task Group 10.

[65] K. K. Shung, M. B. Smith, and B. Tsui, Principles of Medical Imaging. Waltham, MA: Academic Press, 1992.

[66] B. K. Stewart and H. K. Huang, "Single-exposure dual-energy computed radiography," Medical Physics, vol. 17, no. 5, pp. 866-875, 1990.

[67] G-1092: Rotating anode X-ray tube, Varian medical systems, 2007, data sheet.

[68] J. C. Wandtke, "Bedside chest radiography," Radiology, vol. 190, pp. 1-10, 1994.

[69] J. B. Weaver and A. L. Huddleston, "Attenuation coefficients of body tissues using principal-components analysis," Medical Physics, vol. 12, no. 1, pp. 40-45, 1985.

[70] A. J. Wilson and O. C. West, "Single-exposure conventional and computed radiography: the hybrid cassette revisited." Investigative Radiology, vol. 28, pp. 409-412, 1993.

[71] M. J. Yaffe and J. A. Rowlands, "X-ray detectors for digital radiography," Physics in Medicine and Biology, vol. 42, pp. 1-39, 1996.

[72] W. Zhao, K. P. Andriole, and E. Samei, Advances in Medical Physics. Madison, WI: Medical Physics Publishing, 2006, ch. Digital radiography and fluoroscopy. 


\section{Appendix A}

\section{Calibration Phantoms Drawings}

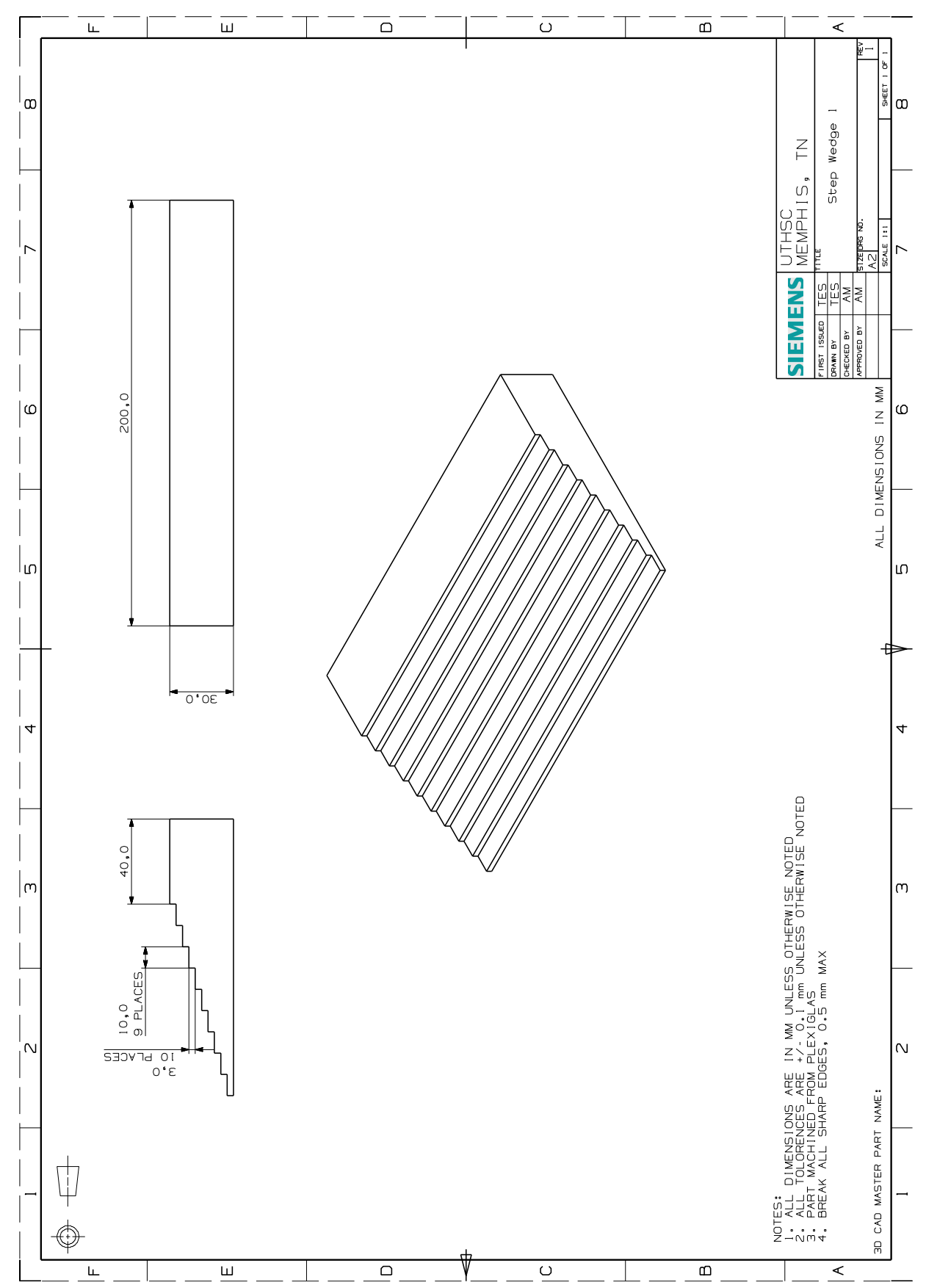




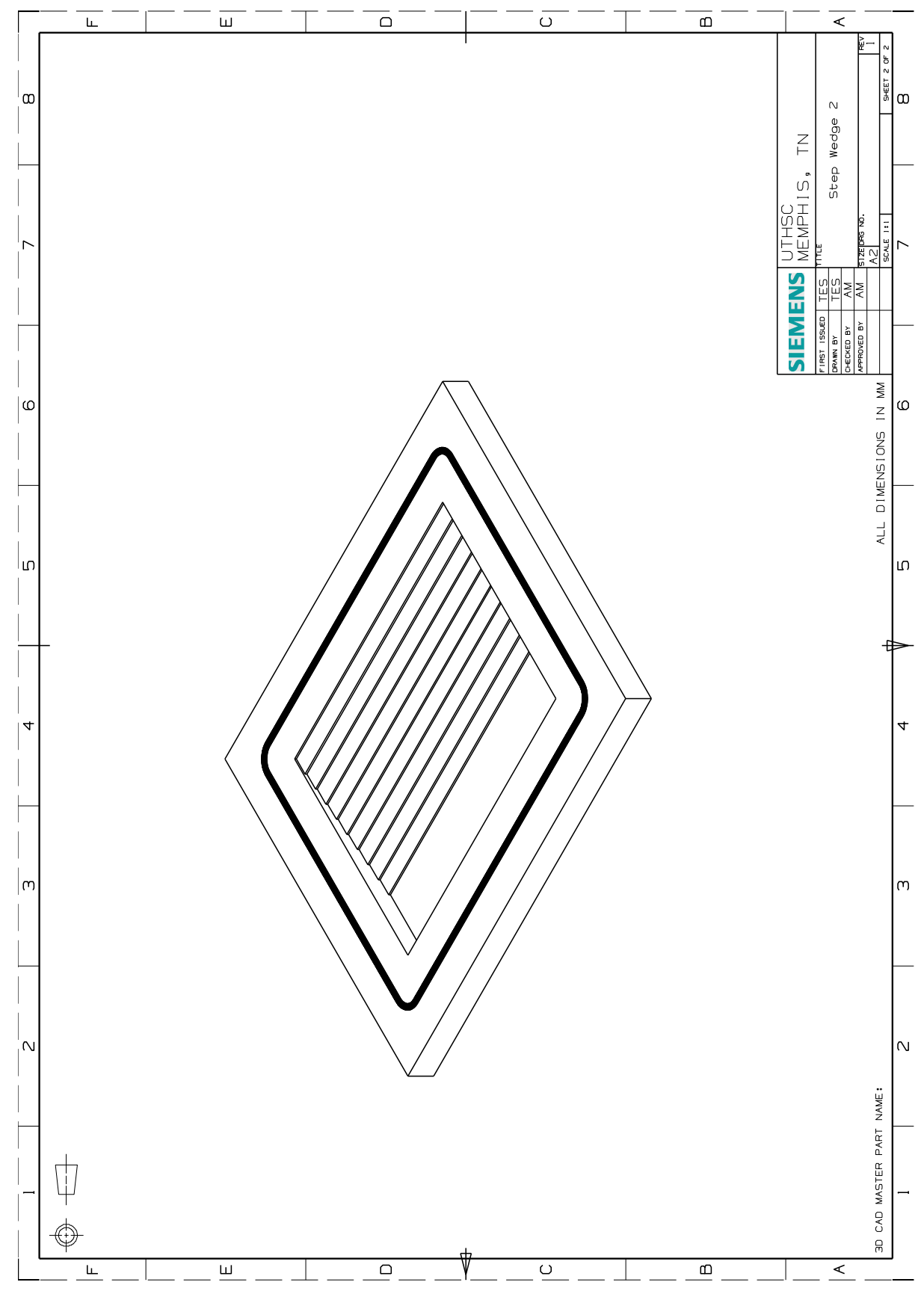




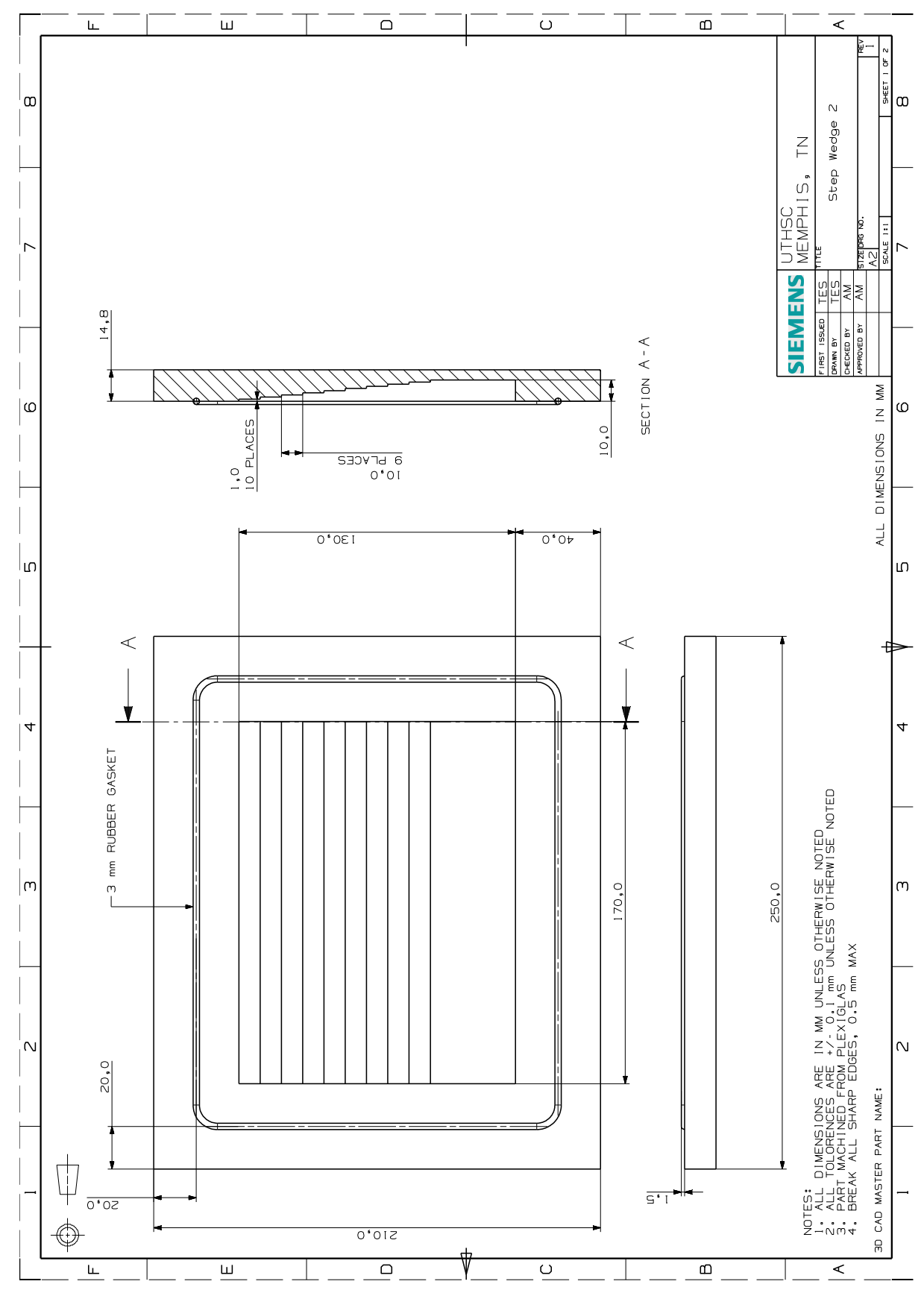




\section{Appendix B}

\section{Test Phantoms Drawings}

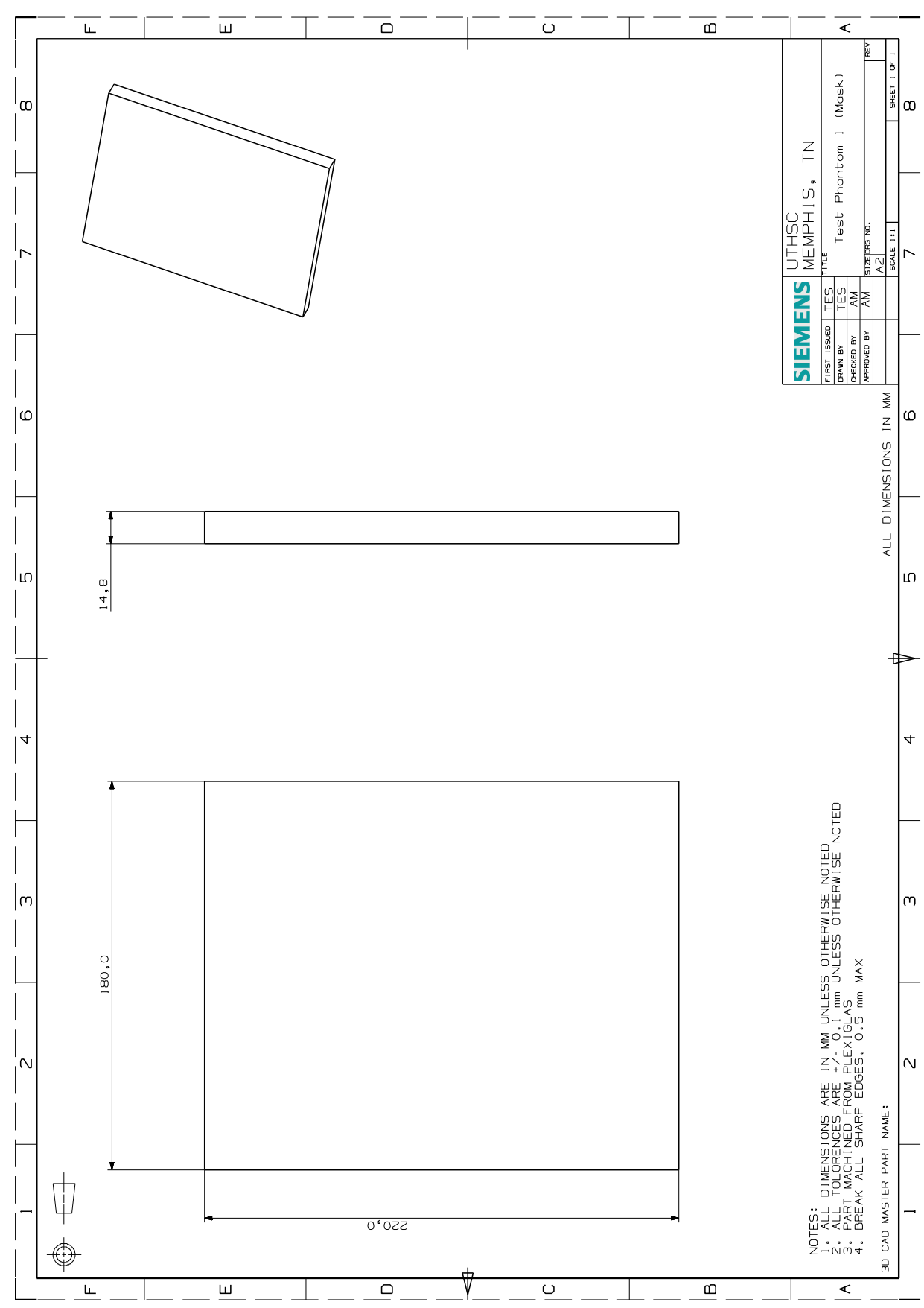




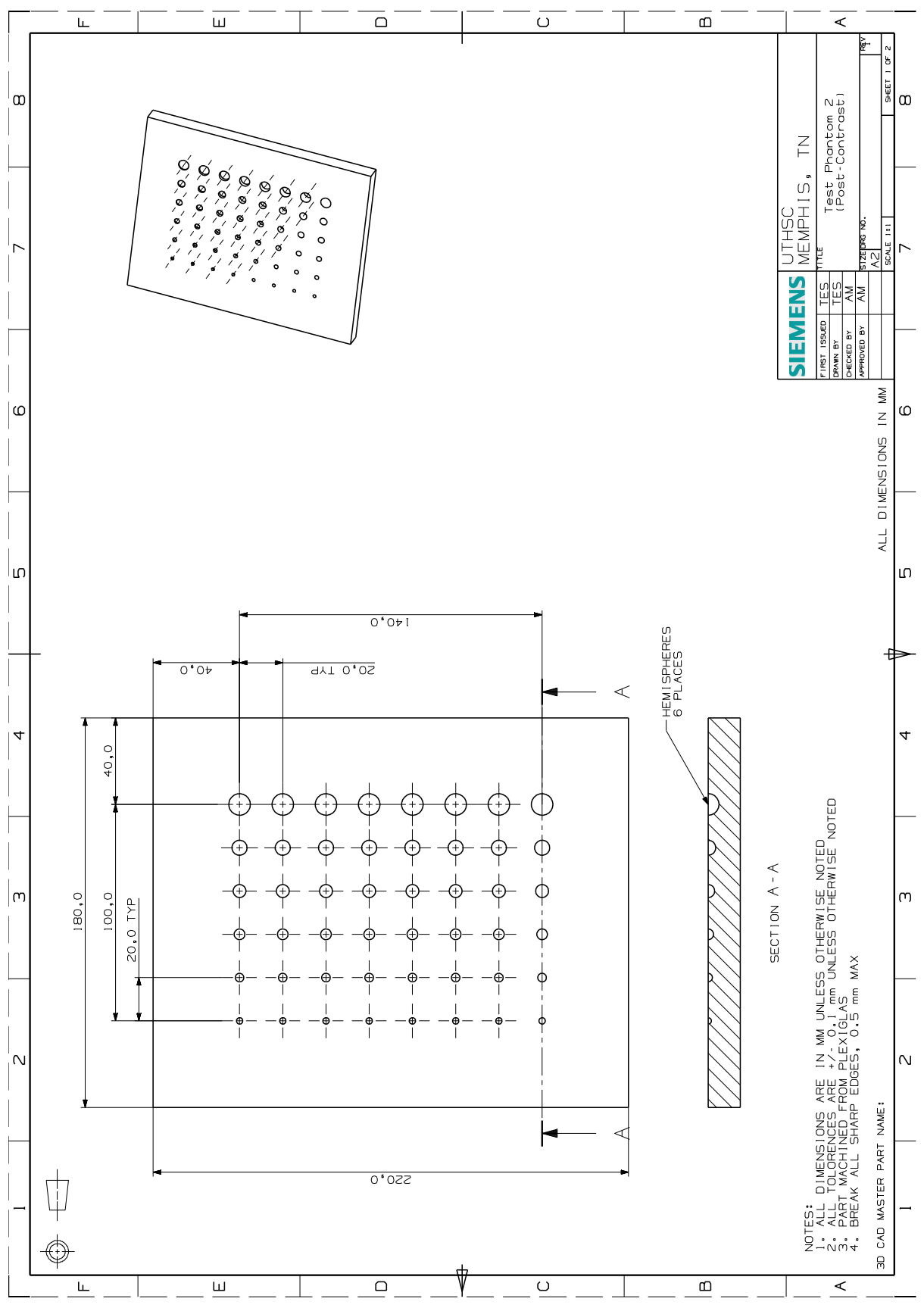




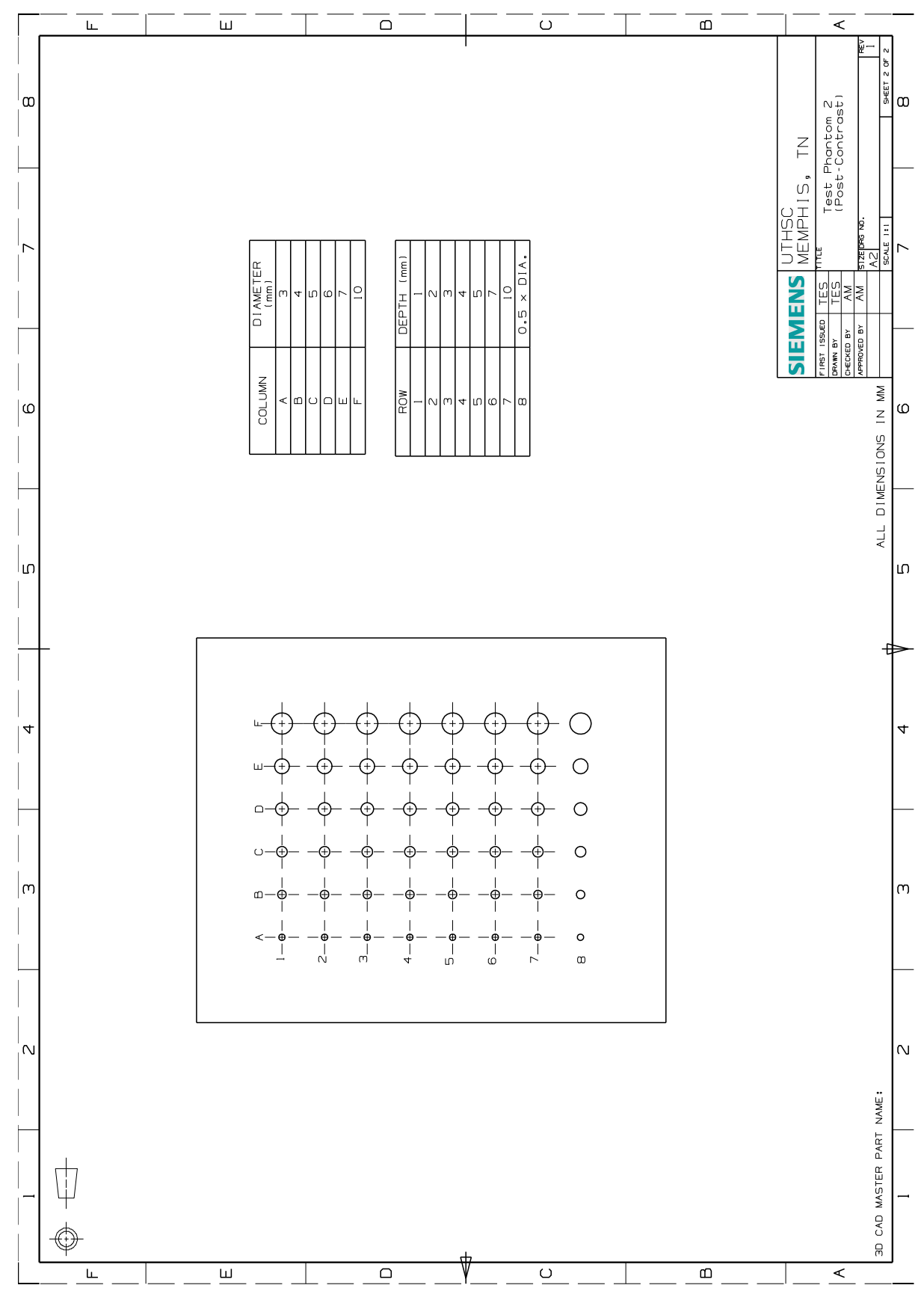




\section{Vita}

Anobel Maghsoodpour was born in Tehran, Iran in 1977. In 2001, he graduated from Tehran Azad University with a Bachelor of Science degree in Biomedical Engineering. He worked as a Service Engineer, Application Engineer, and Quality Engineer in the Medical Instruments industry through 2003. He entered the California State University of Northridge, majoring in Electrical Engineering and graduated with a Master of Science degree in 2005. He pursued his graduate study in the Biomedical Engineering and Imaging program of the University of Tennessee Health Science Center and received his Doctor of Philosophy degree in 2011. His research interest in biomedical imaging mainly involved small animal X-ray imaging, in particular study the application of a low-cost high-resolution computed radiography system in detection of an iodinated contrast agent. 